\title{
Functionalized Heteroaryl-pyridazines and Pyridazin-3(2H)-one Derivatives via Palladium-Catalyzed Cross-Coupling Methodology
}

Kate M. Clapham, ${ }^{\dagger}$ Andrei S. Batsanov, ${ }^{\dagger}$ Ryan D. R. Greenwood, ${ }^{\dagger}$ Martin R. Bryce ${ }^{*} \dagger$ Amy E. Smith, $\dagger, \ddagger$ and Brian Tarbit $\$$

$\dagger$ Department of Chemistry, University of Durham, Durham, DH1 3LE, England and $\$$ Vertellus Specialties UK Ltd., Seal Sands Road, Middlesbrough, TS2 1UB, England.

\section{Supporting Information}

\section{Contents}

Page S2 General Considerations

Pages S3-S14 Synthetic details and characterization data for compounds 1, 10-16, 18a, c, d, 19a, c, d, 20a, c, d, 21-32, 34, 36-38, 39 and $1 \cdot B(\mathrm{OH})_{3}$.

Pages S15-S18 X-Ray crystallographic data for 20b, 20d, 22, 24, 38, 39 and $\mathbf{1} \cdot \mathrm{B}(\mathrm{OH})_{3}$.

Pages S19-S54 Copies of NMR spectra

Page S54 References for Supporting Information 


\section{General Considerations}

General details of equipment and techniques used are the same as those we have reported previously. ${ }^{1}$ All reactions were carried out under an argon atmosphere, glassware was either flame dried or dried in the oven prior to use. All reactions were set up outside of a glovebox, including the weighing of solid substrates. $\mathrm{Pd}(\mathrm{PPh})_{3} \mathrm{Cl}_{2}$ was supplied from Vertellus Specialties UK Ltd or prepared in house, ${ }^{2} \mathrm{Pd}(\mathrm{OAc})_{2}$ was purchased from Aldrich, $\mathrm{Pd}_{2}(\mathrm{dba})_{3}$ was purchased from Alfa Aesar. $t-\mathrm{Bu}_{3} \mathrm{P}$ and $\mathrm{D}-\mathrm{t}-\mathrm{BPF}$ were purchased from Strem, $\mathrm{PCy}_{3}$ was purchased from Molekula. All other reagents employed were of standard reagent grade, purchased from either Aldrich or Alfa Aesar and used without further purification. Anhydrous solvents were dried through a HPLC column on an Innovative Technology Inc. solvent purification system. All other solvents in this work were used without prior purification. Column chromatography was carried out using 40-60 $\mu \mathrm{m}$ mesh silica (Fluorochem). Thin-layer chromatography (TLC) was performed on $20 \mathrm{~mm}$ precoated plates of silica gel (Merck, silica gel $60 \mathrm{~F}_{254}$ ), visualisation was made using ultraviolet light $(254 \mathrm{~nm})$. NMR spectra were recorded on Bruker Avance-400 ( ${ }^{1} \mathrm{H}$ NMR (400 MHz), ${ }^{13} \mathrm{C}$ NMR $(100 \mathrm{MHz}),{ }^{11} \mathrm{~B}$ NMR $(128 \mathrm{MHz}))$ or Varian $500\left({ }^{13} \mathrm{C}\right.$ NMR $\left.(125 \mathrm{MHz})\right)$ instruments, using deuterated solvent as a lock. Chemical shifts are quoted in ppm, relative to tetramethylsilane (TMS), using TMS or the residual solvent as internal reference for ${ }^{1} \mathrm{H}$ and ${ }^{13} \mathrm{C}$ NMR. Melting points were determined on a Stuart Scientific SMP3 melting point apparatus and are uncorrected. Electron Impact (EI) mass spectra were recorded on a Thermo-Finnigan Trace with positive ionisation mode. Electrospray $\left(\mathrm{ES}^{+}\right)$mass spectra were recorded on a Micromass LCT mass spectrometer. Elemental analyses were obtained on an Exeter analytical Inc. CE-440 elemental analyser. 


\section{3,6-Dimethoxypyridazine (1) ${ }^{3}$}

To a solution of sodium methoxide from sodium $(6.50 \mathrm{~g}, 0.28 \mathrm{~mol})$ in dry methanol $(130 \mathrm{~mL})$, was added a solution of 3,6-dichloropyridazine $(9.70 \mathrm{~g}, 0.065 \mathrm{~mol})$ in anhydrous methanol $(50 \mathrm{~mL})$. The reaction mixture was heated at reflux for $19 \mathrm{~h}$, cooled to room temperature then carefully neutralized with $37 \% \mathrm{HCl}$ aq and evaporated in vacuo to dryness. The solid was extracted with ethyl acetate $(200 \mathrm{~mL})$ and washed twice with deionised water $(2 \times 100 \mathrm{~mL})$. Ethyl acetate was removed to leave 1 as a white crystalline solid (8.47 g, 93\%) which was not further purified: mp 103.6-104.8 ${ }^{\circ} \mathrm{C}$, lit. $\mathrm{mp}^{3} 108{ }^{\circ} \mathrm{C} ;{ }^{1} \mathrm{H}$ NMR (400 MHz, DMSO-d $) \delta 7.22(1 \mathrm{H}, \mathrm{s}), 3.97(3 \mathrm{H}, \mathrm{s}) ;{ }^{13} \mathrm{C} \mathrm{NMR}(100 \mathrm{MHz}$, DMSO-d $\left.{ }_{6}\right), \delta 162.67,122.37,55.02$; MS (EI) m/z 140.1 (M ${ }^{+}, 100 \%$ ). Anal. Calcd. for $\mathrm{C}_{6} \mathrm{H}_{8} \mathrm{~N}_{2} \mathrm{O}_{2}$ : C, 51.42; H, 5.75; N, 19.19. Found: C, 51.30; H, 5.78; N, 19.48\%.

\section{2-(3,6-Dimethoxypyridazin-4-yl)-5-nitropyridine (10)}

Compound 2 (313 mg, 1.7 mmol), 2-bromo-5-nitropyridine 3 (305 mg, 1.5 mmol), Pd(OAc) 2 (19.0 mg, $0.085 \mathrm{mmol}$ ), D-t-BPF (38.0 mg, $0.080 \mathrm{mmol})$, 1,4-dioxane $(10 \mathrm{~mL})$ and $\mathrm{Na}_{2} \mathrm{CO}_{3}(1 \mathrm{M}, 4 \mathrm{~mL})$; reaction time $65 \mathrm{~h}$; eluent EtOAc gave 10 as a light brown crystalline solid (353 mg, 90\%): $\mathrm{mp}$ 144.4-145.9 ${ }^{\circ} \mathrm{C} ;{ }^{1} \mathrm{H}$ NMR $\left(400 \mathrm{MHz}, \mathrm{CDCl}_{3}\right) \delta 9.51(1 \mathrm{H}, \mathrm{d}, J=2.4 \mathrm{~Hz}), 8.57(1 \mathrm{H}, \mathrm{dd}, J=11.2 \mathrm{~Hz}, J$ $=2.8 \mathrm{~Hz}), 8.37(1 \mathrm{H}, \mathrm{d}, J=8.8 \mathrm{~Hz}), 7.74(1 \mathrm{H}, \mathrm{s}), 4.21(3 \mathrm{H}, \mathrm{s}), 4.12(3 \mathrm{H}, \mathrm{s}) ;{ }^{13} \mathrm{C} \mathrm{NMR}(100 \mathrm{MHz}$, $\left.\left.\mathrm{CDCl}_{3}\right), \delta 163.4,158.8,156.1,145.1,143.9,131.7,129.4,125.0,121.0,55.3,55.0 ; \mathrm{MS}_{(\mathrm{ES}}{ }^{+}\right) \mathrm{m} / \mathrm{z}$ $263.1\left([\mathrm{M}+1]^{+}, 100 \%\right)$. Anal. Calcd. for $\mathrm{C}_{11} \mathrm{H}_{10} \mathrm{~N}_{4} \mathrm{O}_{4}$ : C, 50.38; H, 3.84; N, 21.37. Found: C, 50.36; H, 3.84; N, 21.27\%.

\section{2-Amino-3-nitro-5-(3,6-dimethoxypyridazin-4-yl)-pyridine (11)}

Compound 2 (313 mg, $1.7 \mathrm{mmol}$ ), 2-amino-5-bromo-3-nitropyridine 4 (327 mg, $1.5 \mathrm{mmol}$ ), $\mathrm{Pd}(\mathrm{OAc})_{2}(19.0 \mathrm{mg}, 0.085 \mathrm{mmol}), \mathrm{D}-\mathrm{t}-\mathrm{BPF}(38.0 \mathrm{mg}, 0.080 \mathrm{mmol}), 1,4-$ dioxane $(10 \mathrm{~mL})$ and $\mathrm{Na}_{2} \mathrm{CO}_{3}(1 \mathrm{M}, 4 \mathrm{~mL})$; reaction time $65 \mathrm{~h}$; eluent EtOAc gave 11 as a yellow crystalline solid (288 mg, 69\%): mp 235.0-236.2 ${ }^{\circ} \mathrm{C} ;{ }^{1} \mathrm{H}$ NMR (400 MHz, DMSO-d 6 ) $\delta 8.75$ (2H, s), 8.23 (2H, s), 7.45 $(1 \mathrm{H}, \mathrm{s}), 4.02(3 \mathrm{H}, \mathrm{s}), 3.98(3 \mathrm{H}, \mathrm{s}) ;{ }^{13} \mathrm{C} \mathrm{NMR}\left(100 \mathrm{MHz}, \mathrm{DMSO}-\mathrm{d}_{6}\right) \delta 162.3,158.6,156.1,153.5$,

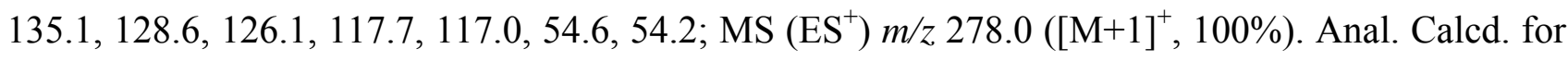
$\mathrm{C}_{11} \mathrm{H}_{11} \mathrm{~N}_{5} \mathrm{O}_{4}$ : C, 47.66; H, 4.00; N, 25.26. Found: C, 47.47; H, 3.91; N, 24.90\%.

\section{5-(3,6-Dimethoxypyridazin-4-yl)-pyrimidine (12)}

Compound 2 (313 mg, $1.7 \mathrm{mmol}$ ), 5-bromopyrimidine 5 (238 mg, $1.5 \mathrm{mmol}), \mathrm{Pd}\left(\mathrm{PhCN}_{2} \mathrm{Cl}_{2}(33.0\right.$ $\mathrm{mg}, 0.085 \mathrm{mmol}), t-\mathrm{Bu}_{3} \mathrm{P}(0.02 \mathrm{~mL}, 0.080 \mathrm{mmol}), 1,4$-dioxane $(10 \mathrm{~mL})$ and $\mathrm{Na}_{2} \mathrm{CO}_{3}(1 \mathrm{M}, 4 \mathrm{~mL})$; reaction time $65 \mathrm{~h}$; eluent EtOAc gave 12 as a yellow solid $(258 \mathrm{mg}, 78 \%)$ : mp 168.8-169.5 ${ }^{\circ} \mathrm{C} ;{ }^{1} \mathrm{H}$ NMR (400 MHz, $\left.\mathrm{CDCl}_{3}\right) \delta 9.26(1 \mathrm{H}, \mathrm{s}), 8.99(2 \mathrm{H}, \mathrm{s}), 7.01(1 \mathrm{H}, \mathrm{s}), 4.11(3 \mathrm{H}, \mathrm{s}), 4.09(3 \mathrm{H}, \mathrm{s}) ;{ }^{13} \mathrm{C}$ NMR $\left(100 \mathrm{MHz}, \mathrm{CDCl}_{3}\right) \delta 162.6,159.0,158.9,156.7,127.9,127.6,119.4,55.3,55.0 ; \mathrm{MS}\left(\mathrm{ES}^{+}\right) \mathrm{m} / \mathrm{z}$ 
$219.4\left([\mathrm{M}+1]^{+}, 100 \%\right)$; HRMS (EI) calcd for $\mathrm{C}_{10} \mathrm{H}_{10} \mathrm{~N}_{4} \mathrm{O}_{2}$ 218.0804, found: 218.0806. Anal. Calcd. for $\mathrm{C}_{10} \mathrm{H}_{10} \mathrm{~N}_{4} \mathrm{O}_{2}$ : C, 55.04; H, 4.62; N, 25.68. Found: C, 54.72; H, 4.61; N, 25.24\%.

\section{2-Amino-5-(3,6-dimethoxypyridazin-4-yl)-pyrimidine (13)}

Compound 2 (313 mg, $1.7 \mathrm{mmol}$ ), 2-amino-5-bromopyrimidine 6 (261 mg, $1.5 \mathrm{mmol}$ ), Pd(OAc) 2 (19.0 mg, $0.085 \mathrm{mmol})$, D-t-BPF (38.0 mg, $0.080 \mathrm{mmol})$, 1,4-dioxane (10 mL) and $\mathrm{Na}_{2} \mathrm{CO}_{3}(1 \mathrm{M}, 4$ $\mathrm{mL}$ ); reaction time $65 \mathrm{~h}$; eluent EtOAc gave 13 as a beige solid $(234 \mathrm{mg}, 65 \%)$ : $\mathrm{mp}$ 253.2-254.2 ${ }^{\circ} \mathrm{C}$; ${ }^{1} \mathrm{H}$ NMR $\left(400 \mathrm{MHz}, \mathrm{CDCl}_{3}\right) \delta 8.61(2 \mathrm{H}, \mathrm{s}), 6.92(1 \mathrm{H}, \mathrm{s}), 5.46(2 \mathrm{H}, \mathrm{s}), 4.10(3 \mathrm{H}, \mathrm{s}), 7.08(3 \mathrm{H}, \mathrm{s}) ;{ }^{13} \mathrm{C}$ NMR $\left.\left(100 \mathrm{MHz}, \mathrm{CDCl}_{3}\right) \delta 163.0,162.7,159.2,158.5,128.7,117.6,117.2,55.1,54.8 ; \mathrm{MS}_{(\mathrm{ES}}{ }^{+}\right) \mathrm{m} / \mathrm{z}$ $234.1\left([\mathrm{M}+1]^{+}, 100 \%\right)$. Anal. Calcd. for $\mathrm{C}_{10} \mathrm{H}_{11} \mathrm{~N}_{5} \mathrm{O}_{2}$ : C, 51.50; H, 4.75; N, 30.03. Found: C, 51.41; H, 4.75; N, 29.97\%.

\section{2-Amino-5-(3,6-dimethoxypyridazin-4-yl)-pyrazine (14)}

Compound 2 (313 mg, $1.7 \mathrm{mmol}$ ), 2-amino-5-bromopyrazine 7 (281 mg, $1.5 \mathrm{mmol}), \mathrm{Pd}(\mathrm{PhCN})_{2} \mathrm{Cl}_{2}$ (33.0 mg, $0.085 \mathrm{mmol}), t-\mathrm{Bu}_{3} \mathrm{P}(0.02 \mathrm{~mL}, 0.080 \mathrm{mmol}), 1,4$-dioxane $(10 \mathrm{~mL})$ and $\mathrm{Na}_{2} \mathrm{CO}_{3}(4 \mathrm{~mL}, 1$ $\mathrm{M}$ ); reaction time $65 \mathrm{~h}$; recrystallized from toluene to give $\mathbf{1 4}$ as a yellow/orange solid (168 $\mathrm{mg}$, 48\%): mp 233.3-235.4 ${ }^{\circ} \mathrm{C}$; ${ }^{1} \mathrm{H}$ NMR (400 MHz, DMSO-d 6 ) $\delta 8.72(1 \mathrm{H}, \mathrm{d}, J=1.2 \mathrm{~Hz}), 8.03(1 \mathrm{H}, \mathrm{d}, J$ $=1.2 \mathrm{~Hz}), 7.48(1 \mathrm{H}, \mathrm{s}), 7.04(2 \mathrm{H}, \mathrm{s}), 4.07(3 \mathrm{H}, \mathrm{s}), 3.97(3 \mathrm{H}, \mathrm{s}) ;{ }^{13} \mathrm{C}$ NMR $\left(100 \mathrm{MHz}, \mathrm{DMSO}-\mathrm{d}_{6}\right), \delta$ $162.6,158.4,155.8,143.5,132.7,132.1,129.6,115.4,54.5,54.2 ; \mathrm{MS}\left(\mathrm{ES}^{+}\right) \mathrm{m} / \mathrm{z} 234.1\left([\mathrm{M}+1]^{+}\right.$, 100\%). Anal. Calcd. for $\mathrm{C}_{10} \mathrm{H}_{11} \mathrm{~N}_{5} \mathrm{O}_{2}$ : C, 51.50; H, 4.75; N, 30.03. Found: C, 51.21; H, 4.74; N, $29.73 \%$.

\section{2-Nitro-5-(3,6-dimethoxypyridazin-4-yl)-thiophene (15)}

Compound 2 (313 mg, 1.7 mmol), 2-bromo-5-nitrothiophene 8 (312 mg, 1.5 mmol), Pd(OAc) 2 (19.0 $\mathrm{mg}, 0.085 \mathrm{mmol})$, D-t-BPF (38.0 mg, $0.080 \mathrm{mmol}), 1,4$-dioxane $(10 \mathrm{~mL})$ and $\mathrm{Na}_{2} \mathrm{CO}_{3}(1 \mathrm{M}, 4 \mathrm{~mL})$; reaction time $65 \mathrm{~h}$; eluent EtOAc gave 15 as a brown powder $(309 \mathrm{mg}, 77 \%)$ : $\mathrm{mp} 185.1-186.9{ }^{\circ} \mathrm{C} ;{ }^{1} \mathrm{H}$ NMR (400 MHz, $\left.\mathrm{CDCl}_{3}\right) \delta 7.94(1 \mathrm{H}, \mathrm{d}, J=4.4 \mathrm{~Hz}), 7.64(1 \mathrm{H}, \mathrm{d}, J=4.0 \mathrm{~Hz}), 7.24(1 \mathrm{H}, \mathrm{s}), 4.26(3 \mathrm{H}$, s), $4.12(3 \mathrm{H}, \mathrm{s}) ;{ }^{13} \mathrm{C} \mathrm{NMR}\left(100 \mathrm{MHz}, \mathrm{CDCl}_{3}\right) \delta 162.7,157.2,140.6,128.1,127.1,124.6,115.9$, 55.4, 55.1; MS $\left(\mathrm{ES}^{+}\right) \mathrm{m} / \mathrm{z} 268.1\left([\mathrm{M}+1]^{+}, 100 \%\right)$. HRMS $\left(\mathrm{ES}^{+}\right) \mathrm{m} / \mathrm{z}$ calcd for $\mathrm{C}_{10} \mathrm{H}_{10} \mathrm{~N}_{3} \mathrm{O}_{4} \mathrm{~S}$ : 268.0392. Found: 268.0387. Anal. Calcd. for $\mathrm{C}_{10} \mathrm{H}_{9} \mathrm{~N}_{3} \mathrm{O}_{4} \mathrm{~S}$ : C, 44.94; H, 3.39; N, 15.72. Found: C, 44.79; H, 3.47; N, 15.01\%.

\section{3-(3,6-Dimethoxypyridazin-4-yl)-quinoline (16)}

Compound 2 (313 mg, $1.7 \mathrm{mmol})$, 3-bromoquinoline 9 (0.2 mL, $1.5 \mathrm{mmol}), \mathrm{Pd}(\mathrm{OAc})_{2}(19.0 \mathrm{mg}$, $0.085 \mathrm{mmol})$, D-t-BPF (38.0 mg, $0.080 \mathrm{mmol}), 1$,4-dioxane $(10 \mathrm{~mL}), \mathrm{Na}_{2} \mathrm{CO}_{3}(1 \mathrm{M}, 4 \mathrm{~mL})$; reaction time $65 \mathrm{~h}$; eluent EtOAc gave 16 as a white solid (381 mg, 95\%): mp 146.6-147.1 ${ }^{\circ} \mathrm{C} ;{ }^{1} \mathrm{H}$ NMR (400 
MHz, $\mathrm{d}_{6}$-acetone) $\delta 9.22(1 \mathrm{H}, \mathrm{d}, J=2.0 \mathrm{~Hz}), 8.69(1 \mathrm{H}, \mathrm{d}, J=2.8 \mathrm{~Hz}), 8.14(1 \mathrm{H}, \mathrm{d}, J=9.2 \mathrm{~Hz}), 8.08$ $(1 \mathrm{H}, \mathrm{d}, J=9.6 \mathrm{~Hz}), 7.88(1 \mathrm{H}, \mathrm{m}), 7.71(1 \mathrm{H}, \mathrm{m}), 7.34(1 \mathrm{H}, \mathrm{s}), 4.13(3 \mathrm{H}, \mathrm{s}), 4.10(3 \mathrm{H}, \mathrm{s}) ;{ }^{13} \mathrm{C} \mathrm{NMR}$ (100 MHz, $\mathrm{d}_{6}$-acetone), $\delta 163.5,160.2,150.9,148.8,137.1,131.5,131.0,129.9,129.3,128.2,127.9$, 127.7, 120.0, 54.9, 54.6; MS (ES $\left.{ }^{+}\right) \mathrm{m} / \mathrm{z} 268.2\left([\mathrm{M}+1]^{+}, 100 \%\right)$. Calcd for $\mathrm{C}_{15} \mathrm{H}_{13} \mathrm{~N}_{3} \mathrm{O}_{2}$ : C, 67.40; H, 4.90; N, 15.72. Found: C, 67.37; H, 4.85; N, 15.55\%.

\section{General Procedure for the preparation of 18a-d.}

Boronic acid (1.0 equiv.), 3-chloro-6-methoxypyridazine 17 (0.9 equiv.) and $\mathrm{Pd}\left(\mathrm{PPh}_{3}\right)_{2} \mathrm{Cl}_{2}$ (ca. 5 mol\%) were sequentially added to degassed 1,4-dioxane $(100 \mathrm{~mL})$ and the mixture was stirred at 20 ${ }^{\circ} \mathrm{C}$ for 30 min. Degassed aqueous $\mathrm{Na}_{2} \mathrm{CO}_{3}$ solution $(1 \mathrm{M}, 56 \mathrm{~mL})$ was added and the reaction mixture was heated under argon at reflux for $65 \mathrm{~h}$. Solvent was removed in vacuo then ethyl acetate $(50 \mathrm{~mL})$ was added and the organic layer was washed with brine $(50 \mathrm{~mL})$, separated, and dried over $\mathrm{MgSO}_{4}$. The mixture was purified by chromatography on a silica gel column, followed by recrystallization in some cases.

\section{3-Methoxy-6-phenylpyridazine (18a) $)^{4,5}$}

Benzeneboronic acid (2.86 g, $0.024 \mathrm{~mol})$, 3-chloro-6-methoxypyridazine 17 (3.0 g, $0.021 \mathrm{~mol})$, $\mathrm{Pd}\left(\mathrm{PPh}_{3}\right)_{2} \mathrm{Cl}_{2}(0.82 \mathrm{~g}, 1.2 \mathrm{mmol})$; eluent petroleum ether $\left(40-60{ }^{\circ} \mathrm{C}\right):$ EtOAc 1:4 v/v, yielded 18a as a white solid (3.68 g, 95\%): mp 119.8-121.4 ${ }^{\circ} \mathrm{C}$ (lit. mp $116{ }^{\circ} \mathrm{C}^{3}$ ); ${ }^{1} \mathrm{H}$ NMR (400 MHz, $\left.\mathrm{CDCl}_{3}\right) \delta 7.97$ $(2 \mathrm{H}, \mathrm{d}, J=8.0 \mathrm{~Hz}), 7.73(1 \mathrm{H}, \mathrm{d}, J=9.2 \mathrm{~Hz}), 7.44(3 \mathrm{H}, \mathrm{m}), 7.00(1 \mathrm{H}, \mathrm{d}, J=9.2 \mathrm{~Hz}), 4.15(3 \mathrm{H}, \mathrm{s}) ;{ }^{13} \mathrm{C}$ NMR (100 MHz, $\left.\mathrm{CDCl}_{3}\right) \delta 164.3,155.2,136.2,129.4,128.9,127.2,126.5,117.7,54.9$; MS (ES $)$ m/z $187.1\left([\mathrm{M}+1]^{+}, 100 \%\right)$. Anal. Calcd. for $\mathrm{C}_{11} \mathrm{H}_{10} \mathrm{~N}_{2} \mathrm{O}: \mathrm{C}, 70.95 ; \mathrm{H}, 5.41 ; \mathrm{N}, 15.04$. Found: C, $70.88 ; \mathrm{H}, 5.37$; N, 15.14\%.

\section{3-Methoxy-6-(2-methoxypyridin-5-yl)-pyridazine (18c)}

2-Methoxy-5-pyridylboronic acid (3.6 g, 0.024 mol), 3-chloro-6-methoxypyridazine 17 (3.0 g, 0.021 mol), $\mathrm{Pd}\left(\mathrm{PPh}_{3}\right)_{2} \mathrm{Cl}_{2}(0.82 \mathrm{~g}, 1.17 \mathrm{mmol})$; eluent EtOAc, yielded 18c as a white solid $(3.85 \mathrm{~g}, 85 \%)$ : mp 146.8-148.5 ${ }^{\circ} \mathrm{C} ;{ }^{1} \mathrm{H}$ NMR $\left(400 \mathrm{MHz}, \mathrm{CDCl}_{3}\right) \delta 8.70(1 \mathrm{H}, \mathrm{d}, J=2.4 \mathrm{~Hz}), 8.31(1 \mathrm{H}, \mathrm{dd}, J=8.6$ $\mathrm{Hz}, J=2.8 \mathrm{~Hz}), 7.73(1 \mathrm{H}, \mathrm{d}, J=9.6 \mathrm{~Hz}), 7.04(1 \mathrm{H}, \mathrm{d}, J=9.6 \mathrm{~Hz}), 6.86(1 \mathrm{H}, \mathrm{dd}, J=9.0 \mathrm{~Hz}, J=0.8$ $\mathrm{Hz}), 4.17(3 \mathrm{H}, \mathrm{s}), 3.99(3 \mathrm{H}, \mathrm{s}) ;{ }^{13} \mathrm{C} \mathrm{NMR}\left(100 \mathrm{MHz}, \mathrm{CDCl}_{3}\right) \delta 165.1,164.4,153.1,145.1,137.0$, 126.4, 125.7, 118.0, 111.4, 55.0, 53.91; $\mathrm{MS}\left(\mathrm{ES}^{+}\right) \mathrm{m} / \mathrm{z} 218.1\left([\mathrm{M}+1]^{+}, 100 \%\right)$. Anal. Calcd. for $\mathrm{C}_{11} \mathrm{H}_{11} \mathrm{~N}_{3} \mathrm{O}_{2}$ : C, 60.82; H, 5.10; N, 19.34. Found: C, 60.73; H, 5.15; N, 19.29\%.

\section{3-Methoxy-6-(2-fluoropyridin-5-yl)-pyridazine (18d)}

2-Fluoro-5-pyridylboronic acid (2.2 g, $0.016 \mathrm{~mol})$, 3-chloro-6-methoxypyridazine 17 (2.0 g, 0.014

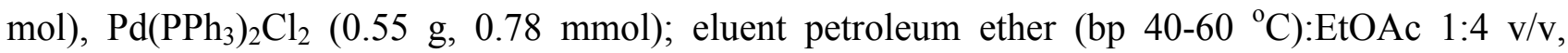


yielded 18d as a pink solid (2.48 g, 87\%): mp 147.7-149.3 ${ }^{\circ} \mathrm{C} ;{ }^{1} \mathrm{H}$ NMR (400 MHz, $\left.\mathrm{CDCl}_{3}\right) \delta 8.75$ $(1 \mathrm{H}, \mathrm{d}, J=2.4 \mathrm{~Hz}), 8.52(1 \mathrm{H}, \mathrm{td}, J=8.2 \mathrm{~Hz}, J=2.4 \mathrm{~Hz}), 7.78(1 \mathrm{H}, \mathrm{d}, J=8.8 \mathrm{~Hz}), 7.09(1 \mathrm{H}, \mathrm{d}, J=$ $9.2 \mathrm{~Hz}), 7.06(1 \mathrm{H}, \mathrm{d}, J=3.2 \mathrm{~Hz}), 4.19(3 \mathrm{H}, \mathrm{s}) ;{ }^{13} \mathrm{C} \mathrm{NMR}\left(100 \mathrm{MHz}, \mathrm{CDCl}_{3}\right) \delta 164.4(1 \mathrm{C}, \mathrm{d}, J=241$ $\mathrm{Hz}), 164.8,152.0,145.8(1 \mathrm{C}, \mathrm{d}, J=15 \mathrm{~Hz}), 139.6$ (1C, d, $J=9 \mathrm{~Hz}), 130.4$ (1C, d, $J=5 \mathrm{~Hz}), 126.7$, 118.2, $110.1(1 \mathrm{C}, \mathrm{d}, J=38 \mathrm{~Hz}), 55.2 ; \mathrm{MS}\left(\mathrm{ES}^{+}\right) \mathrm{m} / \mathrm{z} 206.1\left([\mathrm{M}+1]^{+}, 100 \%\right)$. Anal. Calcd. for $\mathrm{C}_{10} \mathrm{H}_{8} \mathrm{FN}_{3} \mathrm{O}$ : C, 58.53; H, 3.93; N, 20.48. Found: C, 58.25; H, 3.94; N, 20.97\%.

\section{3-Methoxy-6-phenyl-4-pyridazinylboronic acid (19a)}

Diisopropylamine $(3.13 \mathrm{~mL}, 0.022 \mathrm{~mol})$ was added to $n$-butyllithium $(2.5 \mathrm{M}$ in hexane, $8.9 \mathrm{~mL}$, $0.022 \mathrm{~mol})$ in anhydrous THF $(50 \mathrm{~mL})$ at $-78{ }^{\circ} \mathrm{C}$ under argon. The mixture warmed to $0{ }^{\circ} \mathrm{C}$ and left to stir for $0.5 \mathrm{~h}$. The reaction was then cooled to $-78{ }^{\circ} \mathrm{C}$ and a solution of 3-methoxy-6phenylpyridazine 18a $(2.1 \mathrm{~g}, 0.011 \mathrm{~mol})$ in anhydrous THF $(20 \mathrm{~mL})$ was added over $1.5 \mathrm{~h}$. The reaction was stirred for a further $0.5 \mathrm{~h}$. Triisopropylborate $(7.4 \mathrm{~mL}, 0.032 \mathrm{~mol})$ was added at $-78{ }^{\circ} \mathrm{C}$ and the mixture was stirred for $1.5 \mathrm{~h}$ before warming gradually to $-10{ }^{\circ} \mathrm{C}$ when it was quenched with deionised water $(100 \mathrm{~mL})$ and left to stir at room temperature overnight. The organic solvent was removed in vacuo. The resulting aqueous phase was then washed with diethyl ether $(3 \times 50 \mathrm{~mL})$ and treated with $\mathrm{NaOH}$ to obtain $\mathrm{pH} 10$, then acidified to $\mathrm{pH} 6$ using $\mathrm{HBr}$ (48\% aq. solution) to precipitate 19a as a white solid (2.38 g, 96\%): mp 155.6-156.4 ${ }^{\circ} \mathrm{C} ;{ }^{1} \mathrm{H}$ NMR (400 MHz, DMSO-d 6 ) $\delta$ $8.55(2 \mathrm{H}, \mathrm{s}), 8.11(1 \mathrm{H}, \mathrm{s}), 8.06(2 \mathrm{H}, \mathrm{d}, J=6.8 \mathrm{~Hz}), 7.51(3 \mathrm{H}, \mathrm{m}), 4.07(3 \mathrm{H}, \mathrm{s}) ;{ }^{13} \mathrm{C}$ NMR $(100 \mathrm{MHz}$, DMSO-d $\left._{6}\right) \delta 165.5,154.0,136.3,131.1,129.2,128.9,126.2,54.3 ;{ }^{11}$ B NMR $(128 \mathrm{MHz}$, DMSO-d 6 ) $\delta$ 29.8; Anal. Calcd. for $\mathrm{C}_{11} \mathrm{H}_{11} \mathrm{BN}_{2} \mathrm{O}_{3}$ : C, 57.44; H, 4.82; N, 12.18. Found: C, 57.00; H, 4.78; N, $11.82 \%$.

\section{3-Methoxy-6-(2-methoxypyridin-5-yl)-4-pyridazinylboronic acid (19c)}

Diisopropylamine $(1.3 \mathrm{~mL}, 9.2 \mathrm{mmol})$ was added to $n$-butyllithium $(2.5 \mathrm{M}$ in hexane, $3.7 \mathrm{~mL}, 9.2$ mmol) in anhydrous ether $(70 \mathrm{~mL})$ at $-10{ }^{\circ} \mathrm{C}$ under argon. The mixture warmed to $0{ }^{\circ} \mathrm{C}$ and left to stir for $0.5 \mathrm{~h}$. The reaction was then cooled to $-78{ }^{\circ} \mathrm{C}$ and a solution of 3-methoxy-6-(2methoxypyridin-5-yl)-pyridazine 18c $(1.0 \mathrm{~g}, 4.6 \mathrm{mmol})$ in anhydrous ether $(80 \mathrm{~mL})$ was added over $1.25 \mathrm{~h}$. The reaction was stirred for a further $1 \mathrm{~h}$. Triisopropylborate $(3.2 \mathrm{~mL}, 13.8 \mathrm{mmol})$ was added at $-78{ }^{\circ} \mathrm{C}$ and the mixture was stirred for $1.5 \mathrm{~h}$ before warming gradually to room temperature, before quenching with deionised water $(70 \mathrm{~mL})$. After stirring for a further $15 \mathrm{~min}$ the organic solvent was removed in vacuo. The resulting aqueous phase was then washed with diethyl ether $(1 \times$ $150 \mathrm{~mL}$ ) to remove any remaining starting material. The aqueous phase was cooled in an ice bath and acidified with glacial acetic acid to $\mathrm{pH} 5$, where a precipitate formed. The solid was filtered and dried to yield 19c as a pale orange solid (379 mg). TLC analysis of the aqueous phase still showed a baseline spot. The aqueous phase was further acidified to $\mathrm{pH} 3$ and then extracted with EtOAc until a 
TLC of the aqueous phase no longer showed boronic acid. The organic extracts were combined, dried over $\mathrm{MgSO}_{4}$, filtered and evaporated to dryness in vacuo, yielding an orange solid which NMR confirmed to be 19c (352 mg). Total yield of boronic acid 19c (731 mg, 61\%): $\mathrm{mp} 144.4-145.9{ }^{\circ} \mathrm{C}$; ${ }^{1} \mathrm{H}$ NMR $\left(400 \mathrm{MHz}, \mathrm{DMSO}-\mathrm{d}_{6}\right) \delta 8.84(1 \mathrm{H}, \mathrm{d}, J=2.4 \mathrm{~Hz}), 8.56(2 \mathrm{H}, \mathrm{s}), 8.38(1 \mathrm{H}, \mathrm{dd}, J=11.6 \mathrm{~Hz}, J$ $=2.8 \mathrm{~Hz}), 8.12(1 \mathrm{H}, \mathrm{s}), 6.97(1 \mathrm{H}, \mathrm{d}, J=8.8 \mathrm{~Hz}), 4.05(3 \mathrm{H}, \mathrm{s}), 3.92(3 \mathrm{H}, \mathrm{s}) ;{ }^{13} \mathrm{C} \mathrm{NMR}(100 \mathrm{MHz}$, DMSO- $\left.\mathrm{d}_{6}\right) \delta 165.9,164.7,152.4,145.4,137.4,131.0,126.2,111.2,54.8,53.9 ;{ }^{11} \mathrm{~B} \mathrm{NMR}(128 \mathrm{MHz}$, DMSO-d $\left.{ }_{6}\right) \delta 29.3$; Anal. Calcd. for $\mathrm{C}_{11} \mathrm{H}_{12} \mathrm{BN}_{3} \mathrm{O}_{4}$ : C, 50.61; H, 4.63; N, 16.10. Found: C, 50.32; H, $4.60 ; \mathrm{N}, 16.12 \%$.

\section{3-Methoxy-6-(2-fluoropyridin-5-yl)-4-pyridazinylboronic acid (19d)}

Diisopropylamine $(1.4 \mathrm{~mL}, 9.8 \mathrm{mmol})$ was added to $n$-butyllithium $(2.5 \mathrm{M}$ in hexane, $3.9 \mathrm{~mL}, 9.8$ mmol) in anhydrous THF $(50 \mathrm{~mL})$ at $-78{ }^{\circ} \mathrm{C}$ under argon. The mixture warmed to $0{ }^{\circ} \mathrm{C}$ and left to stir for $0.5 \mathrm{~h}$. The reaction was then cooled to $-78{ }^{\circ} \mathrm{C}$ and a solution of 3-methoxy-6-(2fluoropyridin-5-yl)-pyridazine 18d (1.0 g, $4.9 \mathrm{mmol})$ in anhydrous THF (35 mL) was added over 1.5 h. The reaction was stirred for a further $0.5 \mathrm{~h}$. Triisopropylborate $(3.4 \mathrm{~mL}, 14.6 \mathrm{mmol})$ was added at $-78{ }^{\circ} \mathrm{C}$ and the mixture was stirred for $1.5 \mathrm{~h}$ before warming gradually to $-10{ }^{\circ} \mathrm{C}$ when it was quenched with deionised water $(100 \mathrm{~mL})$ and left to stir at room temperature overnight. The organic solvent was removed in vacuo. The resulting aqueous phase was then washed with diethyl ether $(4 \times$ $50 \mathrm{~mL}$ ) and treated with $\mathrm{NaOH}$ to obtain $\mathrm{pH} 10$. The aqueous phase was acidified to $\mathrm{pH} 4 \mathrm{using} \mathrm{HBr}$ ( $48 \%$ aq. solution), and then extracted with ethyl acetate $(4 \times 50 \mathrm{~mL})$. The organic layer was reduced in vacuo and the product 19d was isolated as a light orange solid (0.91 g, 75\%) Recrystallizations were unsuccessful and the product was not further purified: mp ca. $149{ }^{\circ} \mathrm{C}$ (decomp.); ${ }^{1} \mathrm{H}$ NMR (400 MHz, DMSO-d 6 ) $\delta 8.90(1 \mathrm{H}, \mathrm{d}, J=2.8 \mathrm{~Hz}), 8.65(1 \mathrm{H}, \mathrm{td}, J=8.4 \mathrm{~Hz}, J=2.0 \mathrm{~Hz}), 8.60(2 \mathrm{H}, \mathrm{s}), 8.21$ $(1 \mathrm{H}, \mathrm{s}), 7.36(1 \mathrm{H}, \mathrm{dd}, J=9.0 \mathrm{~Hz}, J=2.8 \mathrm{~Hz}), 4.07(3 \mathrm{H}, \mathrm{s}) ;{ }^{13} \mathrm{C}$ NMR $\left(100 \mathrm{MHz}, \mathrm{DMSO}-\mathrm{d}_{6}\right) \delta 166.0$ $(1 \mathrm{C}, \mathrm{d}, J=263 \mathrm{~Hz}), 165.7,151.1,145.5,(1 \mathrm{C}, \mathrm{d}, J=16 \mathrm{~Hz}), 139.9$ (1C, d, $J=9 \mathrm{~Hz}), 131.0,130.6$ $(1 \mathrm{C}, \mathrm{d}, J=4 \mathrm{~Hz}), 109.8(1 \mathrm{C}, \mathrm{d}, J=38 \mathrm{~Hz}), 54.5$; Anal. Calcd. for $\mathrm{C}_{10} \mathrm{H}_{9} \mathrm{BFN}_{3} \mathrm{O}_{3}: \mathrm{C}, 48.23$; H, 3.64; N, 16.88. Found: C, 45.05; H, 3.63; N, 14.60\%. Characterization indicated that 19d is a mixture of boronic acid and anhydride, resulting in the $\mathrm{C}, \mathrm{H}, \mathrm{N}$ analysis being incorrect for pure boronic acid.

\section{General Procedure for the preparation of 20a-d.}

A solution of the boronic acid 19a-d (1.0 equiv.), pinacol (1.0 equiv.) and $\mathrm{MgSO}_{4}$ (2 $\mathrm{g}$ ) in toluene $(25 \mathrm{~mL})$ was stirred for $17 \mathrm{~h}$ at room temperature (TLC monitoring). The suspension was filtered and the resulting solution washed with brine $(3 \times 15 \mathrm{~mL})$. The aqueous phase was washed with $\operatorname{DCM}(1 \times 25 \mathrm{~mL})$. The combined organic layers were dried over $\mathrm{MgSO}_{4}$ and reduced in vacuo to yield pinacol esters 20a-d. 
3-Methoxy-6-phenyl-4-pyridazinylboronic acid 19a (0.350 g, $1.5 \mathrm{mmol})$, pinacol (0.18 g, $1.5 \mathrm{mmol})$, $\mathrm{MgSO}_{4}(2 \mathrm{~g})$, toluene $(25 \mathrm{~mL}) ; 12 \mathrm{~h}$ at room temperature (TLC monitoring); evaporation of the organic layer gave the product 20a as a white solid $(0.378 \mathrm{~g}, 80 \%)$ : mp 151.6-152.8 ${ }^{\circ} \mathrm{C}$; ${ }^{1} \mathrm{H}$ NMR $\left(400 \mathrm{MHz}, \mathrm{CDCl}_{3}\right) \delta 8.10(1 \mathrm{H}, \mathrm{s}), 8.06(2 \mathrm{H}, \mathrm{dd}, J=9.6 \mathrm{~Hz}, J=2.0 \mathrm{~Hz}), 7.47(3 \mathrm{H}, \mathrm{m}), 4.22(3 \mathrm{H}, \mathrm{s})$, $1.39(12 \mathrm{H}, \mathrm{s}) ;{ }^{13} \mathrm{C} \mathrm{NMR}\left(100 \mathrm{MHz}, \mathrm{CDCl}_{3}\right) \delta 166.2,154.9,136.5,133.8,129.4,129.0,126.7,85.0$,

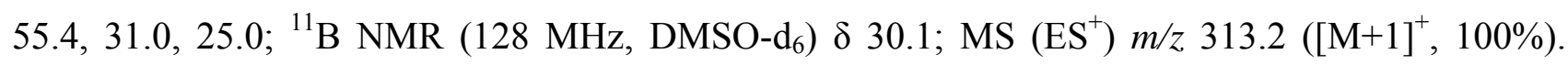
Anal. Calcd. for $\mathrm{C}_{17} \mathrm{H}_{21} \mathrm{BN}_{2} \mathrm{O}_{3}$ : C, 65.41; H, 6.78; N, 8.97. Found: C, 65.09; H, 6.68; N, 8.69\%.

\section{3-Methoxy-6-(2-methoxypyridin-5-yl)-4-(4,4,5,5-tetramethyl-1,3,2-dioxaborolan-2- yl)pyridazine (20c)}

3-Methoxy-6-(2-methoxypyridin-5-yl)-4-pyridazinylboronic acid 19c (0.290 g, $1.1 \mathrm{mmol})$, pinacol $(0.13 \mathrm{~g}, 1.1 \mathrm{mmol}), \mathrm{MgSO}_{4}(2 \mathrm{~g})$, toluene $(10 \mathrm{~mL}) ; 17 \mathrm{~h}$ at room temperature (TLC monitoring); evaporation of the combined organic phase gave the product 20c as a white solid ( $0.337 \mathrm{~g}, 90 \%): \mathrm{mp}$ 146.8-148.2 ${ }^{\circ} \mathrm{C} ;{ }^{1} \mathrm{H}$ NMR (400 MHz, $\left.\mathrm{CDCl}_{3}\right) \delta 8.77(1 \mathrm{H}, \mathrm{dd}, J=2.4 \mathrm{~Hz}, J=0.4 \mathrm{~Hz}), 8.29(1 \mathrm{H}, \mathrm{dd}, J$ $=9.0 \mathrm{~Hz}, J=2.4 \mathrm{~Hz}), 8.03(1 \mathrm{H}, \mathrm{s}), 6.83(1 \mathrm{H}, \mathrm{dd}, J=8.6 \mathrm{~Hz}, J=0.4 \mathrm{~Hz}), 4.19(3 \mathrm{H}, \mathrm{s}), 3.98(3 \mathrm{H}, \mathrm{s})$, $1.36(12 \mathrm{H}, \mathrm{s}) ;{ }^{13} \mathrm{C}$ NMR $\left(100 \mathrm{MHz}, \mathrm{CDCl}_{3}\right) \delta 166.1,165.0,152.7,145.3,136.9,133.1,125.8,111.2$, 85.0, 55.4, 53.8, 24.9; ${ }^{11} \mathrm{~B}$ NMR (128 MHz, $\left.\mathrm{CDCl}_{3}\right) \delta$ 29.97; $\mathrm{MS}\left(\mathrm{ES}^{+}\right) \mathrm{m} / \mathrm{z} 344.3\left([\mathrm{M}+1]^{+}, 100 \%\right)$. Anal. Calcd. for $\mathrm{C}_{17} \mathrm{H}_{22} \mathrm{BN}_{3} \mathrm{O}_{4}$ : C, 59.50; H, 6.46; N, 12.24. Found: C, 58.82; H, 6.47; N, 11.86\%.

\section{3-Methoxy-6-(2-fluoropyridin-5-yl)-4-(4,4,5,5-tetramethyl-1,3,2-dioxaborolan-2-yl)pyridazine} (20d)

3-Methoxy-6-(2-fluoropyridin-5-yl)-4-pyridazinyl boronic acid 19d (0.600 g, $2.4 \mathrm{mmol}$ ), pinacol (0.28 g, $2.4 \mathrm{mmol}), \mathrm{MgSO}_{4}(2 \mathrm{~g})$, toluene $(25 \mathrm{~mL}) ; 12 \mathrm{~h}$ at room temperature (TLC monitoring). evaporation of the combined organic layers yielded 20d as a white solid (0.583 g, 73\%): $\mathrm{mp} 198.2$ $199.7{ }^{\circ} \mathrm{C} ;{ }^{1} \mathrm{H}$ NMR $\left(400 \mathrm{MHz}, \mathrm{CDCl}_{3}\right) \delta 8.78(1 \mathrm{H}, \mathrm{d}, J=2.4 \mathrm{~Hz}), 8.49(1 \mathrm{H}, \mathrm{td}, J=8.0 \mathrm{~Hz}, J=2.4$ $\mathrm{Hz}), 8.04(1 \mathrm{H}, \mathrm{s}), 7.02(1 \mathrm{H}, \mathrm{dd}, J=8.6 \mathrm{~Hz}, J=3.0 \mathrm{~Hz}), 4.18(3 \mathrm{H}, \mathrm{s}), 1.35(12 \mathrm{H}, \mathrm{s}) ;{ }^{13} \mathrm{C}$ NMR $(100$ $\left.\mathrm{MHz}, \mathrm{CDCl}_{3}\right) \delta 166.4,164.2(1 \mathrm{C}, \mathrm{d}, J=242 \mathrm{~Hz}), 151.5,145.8(1 \mathrm{C}, \mathrm{d}, J=16 \mathrm{~Hz}), 139.4(1 \mathrm{C}, \mathrm{d}, J=$ $8 \mathrm{~Hz}), 133.2,130.4(1 \mathrm{C}, \mathrm{d}, J=4 \mathrm{~Hz}), 109.8(1 \mathrm{C}, \mathrm{d}, J=38 \mathrm{~Hz}), 85.1,55.4,24.8,24.6 ;{ }^{11} \mathrm{~B}$ NMR $(128$ $\left.\mathrm{MHz}, \mathrm{DMSO}_{-} \mathrm{d}_{6}\right) \delta 2$ 29.9; MS $\left(\mathrm{ES}^{+}\right) \mathrm{m} / \mathrm{z} 332.2\left([\mathrm{M}+1]^{+}, 100 \%\right)$. HRMS $\left(\mathrm{ES}^{+}\right) \mathrm{m} / \mathrm{z}$ calcd for $\mathrm{C}_{16} \mathrm{H}_{20} \mathrm{BFN}_{3} \mathrm{O}_{3}$ : 332.1576. Found: 332.1573. Anal. Calcd. for $\mathrm{C}_{16} \mathrm{H}_{19} \mathrm{BFN}_{3} \mathrm{O}_{3}: \mathrm{C}, 58.03 ; \mathrm{H}, 5.78 ; \mathrm{N}$, 12.69. Found: C, 56.46; H, 5.76; N, 12.21\%. Crystals for X-ray analysis were grown from toluene/hexane. 


\section{3-Methoxy-6-phenyl-4-(pyridin-2-yl)pyridazine (21)}

Compound 20a (343 mg, $1.1 \mathrm{mmol}), 2$-bromopyridine 33 (0.095 mL, $1.0 \mathrm{mmol}), \operatorname{Pd}_{2}(\mathrm{dba})_{3}(9.2 \mathrm{mg}$, $0.010 \mathrm{mmol}), \mathrm{PCy}_{3}(6.7 \mathrm{mg}, 0.024 \mathrm{mmol}), 1$,4-dioxane $(2.67 \mathrm{~mL})$ and $\mathrm{K}_{3} \mathrm{PO}_{4}(1.27 \mathrm{M}, 1.33 \mathrm{~mL})$; reaction time $24 \mathrm{~h}$; eluent EtOAc:hexane, 2:3 v/v gave 21 as a yellow oil, which solidified on standing (200 mg, 76\%): $\mathrm{mp}$ 68.0-70.6 ${ }^{\circ} \mathrm{C} ;{ }^{1} \mathrm{H}$ NMR (400 MHz, $\left.\mathrm{CDCl}_{3}\right) \delta 8.75(1 \mathrm{H}, \mathrm{d}, J=4.8 \mathrm{~Hz}$ ), $8.54(1 \mathrm{H}, \mathrm{s}), 8.15(1 \mathrm{H}, \mathrm{d}, J=8.4 \mathrm{~Hz}), 8.12,(2 \mathrm{H}, \mathrm{d}, J=8.0 \mathrm{~Hz}), 7.78(1 \mathrm{H}, \mathrm{td}, J=7.6 \mathrm{~Hz}, J=2.0 \mathrm{~Hz})$, $7.47(3 \mathrm{H}, \mathrm{m}), 7.33(1 \mathrm{H}, \mathrm{t}, J=6.2 \mathrm{~Hz}), 4.29(3 \mathrm{H}, \mathrm{s}) ;{ }^{13} \mathrm{C} \mathrm{NMR}\left(100 \mathrm{MHz}, \mathrm{CDCl}_{3}\right) \delta 161.5,156.3$, $151.1,150.1,136.5,136.4,129.4,128.9,127.8,126.7,126.2,125.3,124.1,55.3 ; \mathrm{MS}_{\left(\mathrm{ES}^{+}\right) \mathrm{m} / \mathrm{z} 264.2}$ $\left([\mathrm{M}+1]^{+}, 100 \%\right)$. HRMS $\left(\mathrm{ES}^{+}\right) \mathrm{m} / \mathrm{z}$ calcd for $\mathrm{C}_{16} \mathrm{H}_{14} \mathrm{~N}_{3} \mathrm{O}: 264.1137$. Found: 264.1131. Anal. Calcd. for $\mathrm{C}_{16} \mathrm{H}_{13} \mathrm{~N}_{3} \mathrm{O}$ : C, 72.99; H, 4.98; N, 15.96. Found: C, 72.60; H, 5.06; N, 15.58\%.

Alternative synthesis of $\mathbf{2 1}$ via the organozincate. ${ }^{6}$ A solution of $n$-butyllithium $(2.5 \mathrm{M}$ in hexane, $0.92 \mathrm{~mL}, 2.3 \mathrm{mmol})$, was added to cold $\left(-30{ }^{\circ} \mathrm{C}\right)$ anhydrous THF $(20 \mathrm{~mL})$ under an argon atmosphere. Diisopropylamine $(0.34 \mathrm{~mL}, 2.4 \mathrm{mmol})$ was added and the solution was then stirred at 0 ${ }^{\circ} \mathrm{C}$ for $15 \mathrm{~min}$. The solution was then cooled to $-78{ }^{\circ} \mathrm{C}$ and 3-methoxy-6-phenylpyridazine 18a (186 $\mathrm{mg}, 1.0 \mathrm{mmol})$ dissolved in anhydrous THF $(5 \mathrm{~mL})$ was added dropwise and the mixture was stirred for $1.5 \mathrm{~h}$ at $-78{ }^{\circ} \mathrm{C}$. A solution of zinc chloride (previously dried under vacuum with a heat gun, 272 $\mathrm{mg}, 2.0 \mathrm{mmol})$ dissolved in anhydrous THF $(5 \mathrm{~mL})$ was added to the lithiated pyridazine at $-78{ }^{\circ} \mathrm{C}$, the mixture was allowed to warm to room temperature over $1 \mathrm{~h}$. To the pyridazinylzincate solution, at room temperature, was added $\mathrm{Pd}\left(\mathrm{PPh}_{3}\right)_{4}(4 \mathrm{~mol} \%, 46.2 \mathrm{mg}, 0.04 \mathrm{mmol})$ and 2-bromopyridine 33 $(0.11 \mathrm{~mL}, 1.2 \mathrm{mmol})$, the mixture was fitted with a reflux condenser and heated whilst placed in a sonication bath for $3 \mathrm{~h}$ (the reaction mixture gradually reached reflux whilst under sonication). The mixture was then hydrolysed with a solution of ethylenediamine tetraacetic acid (584 mg, $2.0 \mathrm{mmol}$ ) in deionised water $(10 \mathrm{~mL})$ and made slightly basic with a saturated aqueous solution of potassium carbonate. The aqueous layer was extracted with DCM $(3 \times 25 \mathrm{~mL})$ and the resulting organic layer dried over $\mathrm{MgSO}_{4}$ and concentrated in vacuo. The resulting crude product was purified by column chromatography, eluent EtOAc:petroleum ether (bp 40-60 ${ }^{\circ} \mathrm{C}$ ), 1:4 v/v, yielding 21 as an off-white solid (202 mg, 77\%), spectroscopically identical with the sample described above.

\section{3-Methoxy-6-phenyl-4-(pyrimidin-5-yl)pyridazine (22)}

Compound 20a (343 mg, $1.1 \mathrm{mmol}), 5$-bromopyrimidine 5 (159 mg, $1.0 \mathrm{mmol}), \operatorname{Pd}_{2}(\mathrm{dba})_{3}(9.2 \mathrm{mg}$, $0.010 \mathrm{mmol}), \mathrm{PCy}_{3}(6.7 \mathrm{mg}, 0.024 \mathrm{mmol}), 1,4-$ dioxane $(2.67 \mathrm{~mL})$ and $\mathrm{K}_{3} \mathrm{PO}_{4}(1.27 \mathrm{M}, 1.33 \mathrm{~mL})$; reaction time $24 \mathrm{~h}$; eluent EtOAc gave 22 as an off-white solid (126 mg, 47\%): $\mathrm{mp} \mathrm{165.7-166.3}{ }^{\circ} \mathrm{C}$; ${ }^{1} \mathrm{H}$ NMR $\left(400 \mathrm{MHz}, \mathrm{CDCl}_{3}\right) \delta 9.30(1 \mathrm{H}, \mathrm{s}), 9.08(2 \mathrm{H}, \mathrm{s}), 8.04(2 \mathrm{H}, \mathrm{dd}, J=10 \mathrm{~Hz}, J=2 \mathrm{~Hz}), 7.83$ $(1 \mathrm{H}, \mathrm{s}), 7.50(3 \mathrm{H}, \mathrm{m}), 4.26(3 \mathrm{H}, \mathrm{s}) ;{ }^{13} \mathrm{C} \mathrm{NMR}\left(100 \mathrm{MHz}, \mathrm{CDCl}_{3}\right) \delta 161.2,159.0,156.8,156.2,135.8$, 130.0, 129.2, 128.2, 126.7, 125.5, 123.9, 55.7; MS (EI) m/z 264.0 (M $\left.\mathrm{M}^{+}, 100 \%\right)$. Anal. Calcd. for 
$\mathrm{C}_{15} \mathrm{H}_{12} \mathrm{~N}_{4} \mathrm{O}$ : C, 68.17; H, 4.58; N, 21.20. Found: C, 67.92; H, 4.53; N, 21.23\%. Crystals for X-ray structure determination were grown from EtOAc.

Alternative synthesis of $\mathbf{2 2}$ via the organozincate. The procedure for the preparation of $\mathbf{2 1}$ was followed using $n$-butyllithium $(2.5 \mathrm{M}$ in hexane, $0.92 \mathrm{~mL}, 2.3 \mathrm{mmol})$, diisopropylamine $(0.34 \mathrm{~mL}$, $2.4 \mathrm{mmol}$ ), 3-methoxy-6-phenylpyridazine 18a (186 mg, $1.0 \mathrm{mmol}), \mathrm{ZnCl}_{2}$ (272 mg, $2.0 \mathrm{mmol}$ ), $\mathrm{Pd}\left(\mathrm{PPh}_{3}\right)_{4}(4 \mathrm{~mol} \%, 46.2 \mathrm{mg}, 0.04 \mathrm{mmol})$ and 5-bromopyrimidine 5 (191 mg, $\left.1.2 \mathrm{mmol}\right)$. The mixture was heated and sonicated for $3 \mathrm{~h}$, after which time tlc analysis of the mixture was inconclusive, so heating and sonication were continued for a further $3 \mathrm{~h}$. Chromatography, eluent EtOAc:petroleum ether (bp $40-60{ }^{\circ} \mathrm{C}$ ), 2:3 v/v, gave 22 as a pale yellow solid $(117 \mathrm{mg}, 44 \%$ ), spectroscopically identical with the sample described sbove.

\section{2-Amino-3-nitro-5-(3-methoxy-6-phenylpyridazin-4-yl)-pyridine (23)}

Compound 20a (343 mg, $1.1 \mathrm{mmol}$ ), 2-amino-5-bromo-3-nitropyridine 4 (218 mg, $1.0 \mathrm{mmol}$ ), $\mathrm{Pd}_{2}(\mathrm{dba})_{3}(9.2 \mathrm{mg}, 0.010 \mathrm{mmol}), \mathrm{PCy}_{3}(6.7 \mathrm{mg}, 0.024 \mathrm{mmol})$, 1,4-dioxane $(2.67 \mathrm{~mL})$ and $\mathrm{K}_{3} \mathrm{PO}_{4}$ $(1.27 \mathrm{M}, 1.33 \mathrm{~mL})$; reaction time $24 \mathrm{~h}$; eluent EtOAc:petroleum ether $\left(40-60{ }^{\circ} \mathrm{C}\right) 2: 3 \mathrm{v} / \mathrm{v}$, gave 23 as a yellow solid (190 mg, 59\%): mp 214.6-216.9 ${ }^{\circ} \mathrm{C} ;{ }^{1} \mathrm{H}$ NMR (400 MHz, DMSO-d 6 ) $\delta 8.90(2 \mathrm{H}$, $\mathrm{dd}, J=6.8 \mathrm{~Hz}, J=2.0 \mathrm{~Hz}), 8.33(1 \mathrm{H}, \mathrm{s}), 8.25(2 \mathrm{H}, \mathrm{s}), 8.17(2 \mathrm{H}, \mathrm{d}, J=8.4 \mathrm{~Hz}), 7.52(3 \mathrm{H}, \mathrm{m}), 4.16$ $(3 \mathrm{H}, \mathrm{s}) ;{ }^{13} \mathrm{C}$ NMR $\left(100 \mathrm{MHz}, \mathrm{DMSO}-\mathrm{d}_{6}\right) \delta$ 160.8, 156.4, 155.3, 153.5, 136.0, 135.3, 129.4, 128.8, 126.6, 126.2, 124.9, 124.8, 117.3, 55.0; $\mathrm{MS}\left(\mathrm{ES}^{+}\right) \mathrm{m} / \mathrm{z} 324.2\left([\mathrm{M}+1]^{+}, 100 \%\right)$. Anal. Calcd. for $\mathrm{C}_{16} \mathrm{H}_{13} \mathrm{~N}_{5} \mathrm{O}_{3}$ : C, 59.44; H, 4.05; N, 21.66. Found: C, 59.58; H, 4.04; N, 21.50\%.

\section{3-Methoxy-6-(4-methoxyphenyl)-4-(pyridin-2-yl)pyridazine (24)}

Compound 20b (376 mg, $1.1 \mathrm{mmol})$, 2-bromopyridine 33 (0.095 mL, $1.0 \mathrm{mmol}), \mathrm{Pd}_{2}(\mathrm{dba})_{3}(9.2 \mathrm{mg}$, $0.010 \mathrm{mmol}), \mathrm{PCy}_{3}(6.7 \mathrm{mg}, 0.024 \mathrm{mmol}), 1,4-$ dioxane $(10 \mathrm{~mL})$ and $\mathrm{K}_{3} \mathrm{PO}_{4}(1.27 \mathrm{M}, 1.33 \mathrm{~mL})$; reaction time $24 \mathrm{~h}$; eluent petroleum ether (bp 40-60 ${ }^{\circ} \mathrm{C}$ ):EtOAc, 2:3 v/v, gave 24 as a pale yellow solid (227 mg, 77\%): mp 129.1-131.8 ${ }^{\circ} \mathrm{C} ;{ }^{1} \mathrm{H} \mathrm{NMR}\left(400 \mathrm{MHz}, \mathrm{CDCl}_{3}\right) \delta 8.78(1 \mathrm{H}, \mathrm{d}, J=3.6 \mathrm{~Hz}$ ), $8.50(1 \mathrm{H}, \mathrm{s}), 8.17(1 \mathrm{H}, \mathrm{d}, J=8.0 \mathrm{~Hz}), 8.10(2 \mathrm{H}, \mathrm{dd}, J=7.0 \mathrm{~Hz}, J=2.0 \mathrm{~Hz}), 7.82(1 \mathrm{H}, \mathrm{td}, J=7.8 \mathrm{~Hz}$, $J=1.6 \mathrm{~Hz}), 7.03(2 \mathrm{H}, \mathrm{dd}, J=6.8 \mathrm{~Hz}, J=2.0 \mathrm{~Hz}), 4.30(3 \mathrm{H}, \mathrm{s}), 3.88(3 \mathrm{H}, \mathrm{s}) ;{ }^{13} \mathrm{C} \mathrm{NMR}(100 \mathrm{MHz}$, $\left.\mathrm{CDCl}_{3}\right) \delta 161.3,160.9,156.0,151.3,150.1,136.6,129.0,128.1,127.9,125.7,125.4,124.1,114.4$, 55.5, 55.3; $\mathrm{MS}\left(\mathrm{ES}^{+}\right) \mathrm{m} / \mathrm{z} 294.2\left([\mathrm{M}+1]^{+}, 100 \%\right)$. Anal. Calcd. for $\mathrm{C}_{17} \mathrm{H}_{15} \mathrm{~N}_{3} \mathrm{O}_{2}: \mathrm{C}, 69.61 ; \mathrm{H}, 5.15 ; \mathrm{N}$, 14.33. Found: C, $69.46 ; \mathrm{H}, 5.16 ; \mathrm{N}, 14.39 \%$. Crystals for X-ray structure determination were grown from EtOAc. 
Compound 20b (180 mg, $0.69 \mathrm{mmol})$, 5-bromopyrimidine 5 (100 mg, $0.62 \mathrm{mmol}), \mathrm{Pd}_{2}(\mathrm{dba})_{3}(5.8$ $\mathrm{mg}, 0.0062 \mathrm{mmol}), \mathrm{PCy}_{3}(4.2 \mathrm{mg}, 0.015 \mathrm{mmol}), 1,4$-dioxane $(5 \mathrm{~mL})$ and $\mathrm{K}_{3} \mathrm{PO}_{4}(1.27 \mathrm{M}, 2 \mathrm{~mL})$; microwave heating $120^{\circ} \mathrm{C}, 1 \mathrm{~h}$; eluent EtOAc, gave 25 as a white solid ( $\left.87 \mathrm{mg}, 43 \%\right)$ : $\mathrm{mp} 191.4-$ $192.6{ }^{\circ} \mathrm{C} ;{ }^{1} \mathrm{H}$ NMR $\left(400 \mathrm{MHz}, \mathrm{CDCl}_{3}\right) \delta 9.30(1 \mathrm{H}, \mathrm{s}), 9.07(2 \mathrm{H}, \mathrm{s}), 8.01(2 \mathrm{H}, \mathrm{dd}, J=6.8 \mathrm{~Hz}, J=$ 2Hz), $7.78(1 \mathrm{H}, \mathrm{s}), 7.04(2 \mathrm{H}, \mathrm{dd}, J=6.8 \mathrm{~Hz}, J=2 \mathrm{~Hz}), 4.25(3 \mathrm{H}, \mathrm{s}), 3.88(3 \mathrm{H}, \mathrm{s}) ;{ }^{13} \mathrm{C}$ NMR $(100$ $\left.\mathrm{MHz}, \mathrm{CDCl}_{3}\right) \delta 161.3,160.8,159.0,156.8,155.8,128.34,128.30,128.1,125.1,123.9,114.7,55.58$, 55.56; MS $\left(\mathrm{ES}^{+}\right) \mathrm{m} / \mathrm{z} 295.2\left([\mathrm{M}+1]^{+}, 100 \%\right)$. Anal. Calcd. for $\mathrm{C}_{16} \mathrm{H}_{14} \mathrm{~N}_{4} \mathrm{O}_{2}: \mathrm{C}, 65.30 ; \mathrm{H}, 4.79 ; \mathrm{N}$, 19.04. Found: C, 65.12; H, 4.88; N, 18.66\%.

\section{5-(3-Methoxy-6-(4-methoxyphenyl)pyridazin-4-yl)-3-nitropyridin-2-amine (26)}

Compound 20b (284 mg, $0.83 \mathrm{mmol}$ ), 2-amino-5-bromo-3-nitropyridine 4 (164 mg, $0.75 \mathrm{mmol}$ ), $\mathrm{Pd}_{2}(\mathrm{dba})_{3}(6.9 \mathrm{mg}, 0.0075 \mathrm{mmol}), \mathrm{PCy}_{3}(5.1 \mathrm{mg}, 0.018 \mathrm{~mol}), 1,4$-dioxane $(10 \mathrm{~mL})$ and $\mathrm{K}_{3} \mathrm{PO}_{4}(1.27$ $\mathrm{M}, 1.0 \mathrm{~mL}$ ); reaction time $24 \mathrm{~h}$; eluent petroleum ether (bp 40-60 $\left.{ }^{\circ} \mathrm{C}\right)$ :EtOAc, 2:3 v/v, gave 26 as a yellow solid (120 mg, 46\%): $\mathrm{mp} 222.9-225.8{ }^{\circ} \mathrm{C}$; ${ }^{1} \mathrm{H}$ NMR (400 MHz, DMSO-d $)_{6} \delta 8.89(2 \mathrm{H}, \mathrm{q}, J=$ $6.4 \mathrm{~Hz}, J=2.0 \mathrm{~Hz}), 8.29(1 \mathrm{H}, \mathrm{s}), 8.25(2 \mathrm{H}, \mathrm{s}), 8.14(2 \mathrm{H}, \mathrm{d}, J=8.8 \mathrm{~Hz}), 7.09$ (2H, d, J=8.8 Hz), $4.14(3 \mathrm{H}, \mathrm{s}), 3.84(3 \mathrm{H}, \mathrm{s}) ;{ }^{13} \mathrm{C}$ NMR (100 MHz, DMSO-d 6$) \delta 160.54,160.46,156.5,155.0,153.6$, 135.4, 128.4, 128.0, 125.0, 124.3, 117.4, 114.2, 79.2, 55.3, 54.9: MS (ES $\left.{ }^{+}\right) \mathrm{m} / \mathrm{z} 354.2\left([\mathrm{M}+1]^{+}\right.$, 100\%). Anal. Calcd. for $\mathrm{C}_{17} \mathrm{H}_{15} \mathrm{~N}_{5} \mathrm{O}_{4}: \mathrm{C}, 57.79 ; \mathrm{H}, 4.28 ; \mathrm{N}, 19.82$. Found: C, 58.04; H, 4.25; N, $19.39 \%$.

\section{3-Methoxy-6-(2-methoxypyridin-5-yl)-4-(pyridin-2-yl)pyridazine (27)}

Compound 20c (378 mg, $1.1 \mathrm{mmol})$, 2-bromopyridine $33(0.095 \mathrm{~mL}, 1.0 \mathrm{mmol}), \mathrm{Pd}_{2}(\mathrm{dba})_{3}(9.2 \mathrm{mg}$, $0.010 \mathrm{mmol}), \mathrm{PCy}_{3}(6.7 \mathrm{mg}, 0.024 \mathrm{mmol}), 1,4$-dioxane $(10 \mathrm{~mL})$ and $\mathrm{K}_{3} \mathrm{PO}_{4}(1.27 \mathrm{M}, 1.33 \mathrm{~mL})$; reaction time $24 \mathrm{~h}$; eluent petroleum ether (bp 40-60 ${ }^{\circ} \mathrm{C}$ ):EtOAc 2:3 v/v, gave 27 as a white solid (170 mg, 58\%): mp 156.0-157.7 ${ }^{\circ} \mathrm{C} ;{ }^{1} \mathrm{H}$ NMR (400 MHz, $\left.\mathrm{CDCl}_{3}\right) \delta 8.83(1 \mathrm{H}, \mathrm{d}, J=2.4 \mathrm{~Hz}), 8.72$ $(1 \mathrm{H}, \mathrm{d}, J=4.4 \mathrm{~Hz}), 8.46(1 \mathrm{H}, \mathrm{s}), 8.32(1 \mathrm{H}, \mathrm{dd}, J=9.8 \mathrm{~Hz}, J=2.8 \mathrm{~Hz}), 8.13(1 \mathrm{H}, \mathrm{d}, J=8.0 \mathrm{~Hz}), 7.77$ $(1 \mathrm{H}, \mathrm{td}, J=7.6 \mathrm{~Hz}, J=1.6 \mathrm{~Hz}), 7.32(1 \mathrm{H}, \mathrm{m}), 6.82(1 \mathrm{H}, \mathrm{d}, J=8.0 \mathrm{~Hz}), 4.26(3 \mathrm{H}, \mathrm{s}), 3.96(3 \mathrm{H}, \mathrm{s})$; ${ }^{13} \mathrm{C} \mathrm{NMR}\left(100 \mathrm{MHz}, \mathrm{CDCl}_{3}\right) \delta 165.1,161.6,154.3,151.0,150.2,145.5,137.0,136.6,128.1,125.8$,

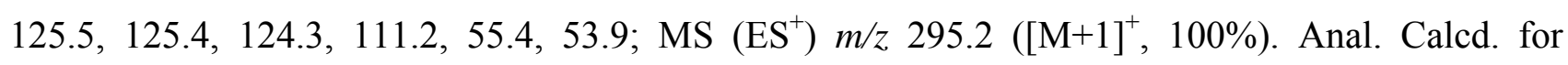
$\mathrm{C}_{16} \mathrm{H}_{14} \mathrm{~N}_{4} \mathrm{O}_{2}$ : C, 65.30; H, 4.79; N, 19.04. Found: C, 65.20; H, 4.83; N, 18.99\%.

Alternative synthesis of $\mathbf{2 7}$ via the organozincate. The procedure for the preparation of $\mathbf{2 1}$ was followed using $n$-butyllithium $(2.5 \mathrm{M}$ in hexane, $0.92 \mathrm{~mL}, 2.3 \mathrm{mmol})$, diisopropylamine $(0.34 \mathrm{~mL}$, $2.4 \mathrm{mmol})$, 3-methoxy-6-(2-methoxypyridin-5-yl)pyridazine 18c (217 mg, $1.0 \mathrm{mmol}), \mathrm{ZnCl}_{2}$ (272 $\mathrm{mg}, 2.0 \mathrm{mmol}), \mathrm{Pd}\left(\mathrm{PPh}_{3}\right)_{4}(4 \mathrm{~mol} \%, 46.2 \mathrm{mg}, 0.04 \mathrm{mmol})$ and 2-bromopyridine $33(0.11 \mathrm{~mL}, 1.2$ $\mathrm{mmol})$. The mixture was heated and sonicated for $4 \mathrm{~h}$. Chromatography, eluent EtOAc:petroleum 
ether $\left(40-60{ }^{\circ} \mathrm{C}\right), 1: 4 \mathrm{v} / \mathrm{v}$ gradient to $1: 1 \mathrm{v} / \mathrm{v}$, gave 27 as an off-white solid $(215 \mathrm{mg}, 73 \%)$ spectroscopically identical with the sample described above.

\section{3-Methoxy-6-(2-methoxypyridin-5-yl)-4-(pyrimidin-5-yl)pyridazine (28)}

Compound 20c (343 mg, $1.0 \mathrm{mmol}$ ), 5-bromopyrimidine 5 (145 mg, $0.91 \mathrm{mmol}), \mathrm{Pd}_{2}(\mathrm{dba})_{3}(8.3 \mathrm{mg}$, $0.0091 \mathrm{mmol}), \mathrm{PCy}_{3}(6.1 \mathrm{mg}, 0.022 \mathrm{mmol}), 1,4$-dioxane $(2.67 \mathrm{~mL})$ and $\mathrm{K}_{3} \mathrm{PO}_{4}(1.27 \mathrm{M}, 1.33 \mathrm{~mL})$; reaction time $24 \mathrm{~h}$; eluent EtOAc, gave 28 as a white solid (102 mg, 37\%): mp 192.0-194.3 ${ }^{\circ} \mathrm{C}$; ${ }^{1} \mathrm{H}$ NMR (400 MHz, CDCl $) \delta 9.29(1 \mathrm{H}, \mathrm{s}), 9.06(2 \mathrm{H}, \mathrm{s}), 8.74(1 \mathrm{H}, \mathrm{d}, J=2.8 \mathrm{~Hz}), 8.31(1 \mathrm{H}, \mathrm{dd}, J=8.8$ $\mathrm{Hz}, J=2.4 \mathrm{~Hz}), 7.76(1 \mathrm{H}, \mathrm{s}), 6.87(1 \mathrm{H}, \mathrm{d}, J=8.8 \mathrm{~Hz}), 4.24(3 \mathrm{H}, \mathrm{s}), 3.99(3 \mathrm{H}, \mathrm{s}) ;{ }^{13} \mathrm{C} \mathrm{NMR}(100$ $\left.\mathrm{MHz}, \mathrm{CDCl}_{3}\right) \delta 165.1,160.9,158.9,156.6,153.8,145.1,136.8,127.8,125.0,124.5,124.0,111.4$, 55.5, 53.8; MS $\left(\mathrm{ES}^{+}\right) \mathrm{m} / \mathrm{z} 296.2\left([\mathrm{M}+1]^{+}, 100 \%\right)$. HRMS $\left(\mathrm{ES}^{+}\right)$calcd for $\mathrm{C}_{15} \mathrm{H}_{14} \mathrm{~N}_{5} \mathrm{O}_{2} 296.1142$ : found 296.1141. Anal. Calcd. for $\mathrm{C}_{15} \mathrm{H}_{13} \mathrm{~N}_{5} \mathrm{O}_{2}$ : C, 61.01; H, 4.44; N, 23.72. Found: C, 60.39; H, $4.39 ; \mathrm{N}, 23.25 \%$.

\section{5-(3-Methoxy-6-(2-methoxypyridin-5-yl)-pyridazin-4-yl)-3-nitropyridin-2-amine (29)}

Compound 20c (337 mg, $0.98 \mathrm{mmol}$ ), 2-amino-5-bromo-3-nitropyridine 4 (195 mg, $0.89 \mathrm{mmol}$ ), $\mathrm{Pd}_{2}(\mathrm{dba})_{3}(8.2 \mathrm{mg}, 0.0089 \mathrm{mmol}), \mathrm{PCy}_{3}(6.0 \mathrm{mg}, 0.021 \mathrm{mmol}), 1,4$-dioxane $(10 \mathrm{~mL})$ and $\mathrm{K}_{3} \mathrm{PO}_{4}$ $(1.27 \mathrm{M}, 1.33 \mathrm{~mL})$; reaction time $24 \mathrm{~h}$; eluent petroleum ether $\left(\mathrm{bp} 40-60{ }^{\circ} \mathrm{C}\right)$ :EtOAc $1: 1 \mathrm{v} / \mathrm{v}$, final elution with EtOAc gave 29 as a yellow solid (134 mg, 42\%): mp 249.5-252.3 ${ }^{\circ} \mathrm{C} ;{ }^{1} \mathrm{H}$ NMR (400 MHz, DMSO-d 6 ) $\delta 8.97(1 \mathrm{H}, \mathrm{d}, J=2.4 \mathrm{~Hz}), 8.91(2 \mathrm{H}, \mathrm{s}), 8.48(1 \mathrm{H}, \mathrm{dd}, J=8.6 \mathrm{~Hz}, J=2.4 \mathrm{~Hz}), 8.38$ $(1 \mathrm{H}, \mathrm{s}), 8.26(2 \mathrm{H}, \mathrm{s}), 7.00(1 \mathrm{H}, \mathrm{d}, J=8.8 \mathrm{~Hz}), 4.16(3 \mathrm{H}, \mathrm{s}), 3.94(3 \mathrm{H}, \mathrm{s}) ;{ }^{13} \mathrm{C} \mathrm{NMR}(100 \mathrm{MHz}$, DMSO-d $\left.{ }_{6}\right) \delta 164.3,160.8,156.5,153.6,153.3,145.5,137.3,135.4,126.2,125.5,125.1,124.4$, 117.2, 110.7, 55.0, 53.5; MS (ES $\left.{ }^{+}\right) \mathrm{m} / \mathrm{z} 355.2\left([\mathrm{M}+1]^{+}, 100 \%\right)$. Anal. Calcd. for $\mathrm{C}_{16} \mathrm{H}_{14} \mathrm{~N}_{6} \mathrm{O}_{4}: \mathrm{C}$, 54.24; H, 3.98; N, 23.72. Found: C, 54.62; H, 4.00; N, 23.43\%.

\section{3-Methoxy-6-(2-fluoropyridin-5-yl)-4-(pyridin-2-yl)pyridazine (30)}

Compound 20d (310 mg, $0.94 \mathrm{mmol}), 2$-bromopyridine $33(0.081 \mathrm{~mL}, 0.85 \mathrm{mmol}), \mathrm{Pd}_{2}(\mathrm{dba})_{3}(7.8$ $\mathrm{mg}, 0.0085 \mathrm{mmol}), \mathrm{PCy}_{3}(5.5 \mathrm{mg}, 0.020 \mathrm{mmol})$, 1,4-dioxane $(2.5 \mathrm{~mL})$ and $\mathrm{K}_{3} \mathrm{PO}_{4}(1.27 \mathrm{M}, 1.1 \mathrm{~mL})$; reaction time $24 \mathrm{~h}$; eluent petroleum ether (bp 40-60 $\left.{ }^{\circ} \mathrm{C}\right)$ :EtOAc 1:1 v/v, gave 30 as an off-white solid (160 mg, 67\%): mp 190.3-191.7 ${ }^{\circ} \mathrm{C} ;{ }^{1} \mathrm{H} \mathrm{NMR}\left(400 \mathrm{MHz}, \mathrm{CDCl}_{3}\right) \delta 8.89(1 \mathrm{H}, \mathrm{d}, J=2.4 \mathrm{~Hz}$ ), $8.75(1 \mathrm{H}, \mathrm{d}, J=4.8 \mathrm{~Hz}), 8.57(1 \mathrm{H}, \mathrm{td}, J=7.8 \mathrm{~Hz}, J=2.4 \mathrm{~Hz}), 8.55(1 \mathrm{H}, \mathrm{s}), 8.19(1 \mathrm{H}, \mathrm{d}, J=8.0 \mathrm{~Hz})$, $7.81(1 \mathrm{H}, \mathrm{td}, J=7.8, J=1.6), 7.37(1 \mathrm{H}, \mathrm{m}), 7.06(1 \mathrm{H}, \mathrm{dd}, J=8.4 \mathrm{~Hz}, J=2.8 \mathrm{~Hz}), 4.31(3 \mathrm{H}, \mathrm{s}) ;{ }^{13} \mathrm{C}$ NMR $\left(100 \mathrm{MHz}, \mathrm{CDCl}_{3}\right) \delta 164.4(1 \mathrm{C}, \mathrm{d}, J=242 \mathrm{~Hz}), 161.9,153.0,150.5,150.2,146.0(1 \mathrm{C}, \mathrm{d}, J=$ $15 \mathrm{~Hz}), 139.5$ (1C, d, $J=8 \mathrm{~Hz}), 136.7,130.5$ (1C, d, $J=4 \mathrm{~Hz}), 128.1,125.6,125.4,124.4,109.9$ $(1 \mathrm{C}, \mathrm{d}, J=38 \mathrm{~Hz}), 55.5$; $\mathrm{MS}\left(\mathrm{ES}^{+}\right) \mathrm{m} / \mathrm{z} 336.9\left([\mathrm{M}+\mathrm{MeOH}+\mathrm{Na}]^{+}, 100 \%\right)$. Anal. Calcd. for $\mathrm{C}_{15} \mathrm{H}_{11} \mathrm{FN}_{4} \mathrm{O}$ : C, 63.83; H, 3.93; N, 19.85. Found: C, 63.58; H, 3.89; N, 19.69\%. 


\section{3-Methoxy-6-(2-fluoropyridin-5-yl)-4-(pyrimidin-5-yl)pyridazine (31)}

Compound 20d (364 mg, $1.1 \mathrm{mmol}), 5$-bromopyrimidine 5 (159 mg, $1.0 \mathrm{mmol}), \operatorname{Pd}_{2}(\mathrm{dba})_{3}(9.2 \mathrm{mg}$, $0.01 \mathrm{mmol}), \mathrm{PCy}_{3}(6.7 \mathrm{mg}, 0.024 \mathrm{mmol}), 1,4$-dioxane $(3 \mathrm{~mL})$ and $\mathrm{K}_{3} \mathrm{PO}_{4}(1.27 \mathrm{M}, 1.3 \mathrm{~mL})$; reaction time $24 \mathrm{~h}$; eluent EtOAc, gave 31 as a white solid (82 mg, 29\%): mp 256.1-257.9 ${ }^{\circ} \mathrm{C} ;{ }^{1} \mathrm{H}$ NMR (400 $\left.\mathrm{MHz}, \mathrm{CDCl}_{3}\right) \delta 9.33(1 \mathrm{H}, \mathrm{s}), 9.09(2 \mathrm{H}, \mathrm{s}), 8.83(1 \mathrm{H}, \mathrm{d}, J=2.5 \mathrm{~Hz}), 8.57(1 \mathrm{H}, \mathrm{td}, J=8.1 \mathrm{~Hz}, J=2.5$ $\mathrm{Hz}), 7.83(1 \mathrm{H}, \mathrm{s}), 7.12(1 \mathrm{H}, \mathrm{dd}, J=8.6 \mathrm{~Hz}, J=3.0 \mathrm{~Hz}), 4.29(3 \mathrm{H}, \mathrm{s}) ;{ }^{13} \mathrm{C} \mathrm{NMR}\left(125 \mathrm{MHz}, \mathrm{CDCl}_{3}\right) \delta$ $164.6(1 \mathrm{C}, \mathrm{d}, J=242 \mathrm{~Hz}), 161.5,159.2,156.8,152.9,145.9$ (1C, d, $J=16 \mathrm{~Hz}), 139.7$ (1C, d, $J=9$ $\mathrm{Hz}), 129.9(1 \mathrm{C}, \mathrm{d}, J=5 \mathrm{~Hz}), 127.8,125.0,124.4,110.3(1 \mathrm{C}, \mathrm{d}, J=37 \mathrm{~Hz}), 55.9$; $\left.\mathrm{MS}_{(\mathrm{ES}}\right)^{+} \mathrm{m} / \mathrm{z}$ $284.2\left([\mathrm{M}+1]^{+}, 100 \%\right)$. Anal. Calcd. for $\mathrm{C}_{14} \mathrm{H}_{10} \mathrm{FN}_{5} \mathrm{O}$ : C, 59.36; H, 3.56; N, 24.72. Found: C, 59.30; $\mathrm{H}, 3.56$; N, 24.45\%.

\section{5-(6-(2-Fluoropyridin-5-yl)-3-methoxypyridazin-4-yl)-3-nitropyridin-2-amine (32)}

Compound 20d (189 mg, $0.57 \mathrm{mmol}$ ), 2-amino-5-bromo-3-nitropyridine 4 (109 mg, $0.5 \mathrm{mmol}$ ), $\mathrm{Pd}(\mathrm{OAc})_{2}(5.6 \mathrm{mg}, 0.025 \mathrm{mmol})$, D-t-BPF (12 mg, $\left.0.025 \mathrm{mmol}\right)$, 1,4-dioxane (7 mL) and $\mathrm{Na}_{2} \mathrm{CO}_{3}(1$ M, $2 \mathrm{~mL}$ ); reaction time $5 \mathrm{~h}$; eluent hexane:EtOAc $1: 1 \mathrm{v} / \mathrm{v}$, recrystallization from EtOAc/hexane, gave 32 as a yellow solid (91 mg, 52\%): $\mathrm{mp} 281.5{ }^{\circ} \mathrm{C}$ (decomp.); ${ }^{1} \mathrm{H}$ NMR (400 MHz, DMSO-d 6 ) $\delta$ $9.01(1 \mathrm{H}, \mathrm{s}), 8.91(2 \mathrm{H}, \mathrm{s}), 8.74(1 \mathrm{H}, \mathrm{t}, J=7.8 \mathrm{~Hz}), 8.45(1 \mathrm{H}, \mathrm{s}), 8.28$ (1H, br. s), 7.34 (1H, d, $J=8.1$ $\mathrm{Hz}), 4.17(3 \mathrm{H}, \mathrm{s}) ;{ }^{13} \mathrm{C}$ NMR $\left(125 \mathrm{MHz}, \mathrm{DMSO}_{-} \mathrm{d}_{6}\right) \delta 163.6(1 \mathrm{C}, \mathrm{d}, J=238 \mathrm{~Hz}), 161.1,156.4,153.6$, 152.3, $146.0(1 \mathrm{C}, \mathrm{d}, J=15 \mathrm{~Hz}), 140.2$, (1C, d, $J=9 \mathrm{~Hz}), 135.4,130.3(1 \mathrm{C}, \mathrm{d}, J=4 \mathrm{~Hz}), 126.2$,

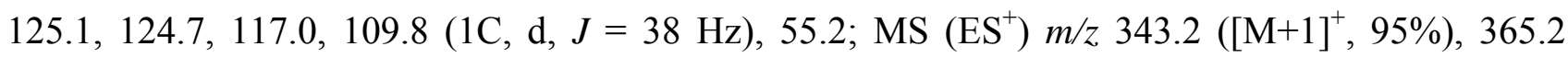
$\left([\mathrm{M}+\mathrm{Na}]^{+}, 100 \%\right)$. HRMS $\left(\mathrm{ES}^{+}\right) \mathrm{m} / \mathrm{z}$ calcd for $\mathrm{C}_{15} \mathrm{H}_{12} \mathrm{~N}_{6} \mathrm{O}_{3} \mathrm{~F}: 343.0949$. Found: 343.0950.

\section{Potassium 3-methoxy-6-phenyl-4-pyridazinyltrifluoroborate salt (34)}

3-Methoxy-6-phenyl-4-pyridazinylboronic acid 19a (640 $\mathrm{mg}, 2.8 \mathrm{mmol})$ and potassium hydrogen fluoride $(650 \mathrm{mg}, 8.3 \mathrm{mmol})$ were stirred in methanol $(5 \mathrm{~mL})$ at room temperature; on addition of water $(3 \mathrm{~mL})$ a white precipitate formed. The reaction mixture was stirred at room temperature for 1 $\mathrm{h}$ before standing in an ice bath for $1 \mathrm{~h}$. The precipitate was filtered and recrystallized from hot $\mathrm{MeCN}$ to yield 34 as a white solid (792 mg, 97\%): $\mathrm{mp}$ 209.4-210.8 ${ }^{\circ} \mathrm{C} ;{ }^{1} \mathrm{H}$ NMR (400 MHz, DMSO$\left.\mathrm{d}_{6}\right) \delta 7.96(2 \mathrm{H}, \mathrm{dd}, J=8.4 \mathrm{~Hz}, J=1.6 \mathrm{~Hz}), 7.82(1 \mathrm{H}, \mathrm{s}), 7.49(2 \mathrm{H}, \mathrm{m}), 7.41(1 \mathrm{H}, \mathrm{m}), 3.97(3 \mathrm{H}, \mathrm{s})$; ${ }^{13} \mathrm{C}$ NMR (125 MHz, DMSO-d 6 ) $\delta 167.8,153.7,137.7,129.6,128.7,128.3,125.9,53.4 ;{ }^{11} \mathrm{~B}$ NMR $\left(128 \mathrm{MHz}, \mathrm{DMSO}-\mathrm{d}_{6}\right) \delta 3.5 ;{ }^{19} \mathrm{~F}$ NMR $\left(376 \mathrm{MHz}, \mathrm{DMSO}-\mathrm{d}_{6}\right) \delta-136.5$.

\section{6-Phenyl-4-(pyrimidin-5-yl)pyridazin-3(2H)-one (37)}

A mixture of 22 (100 mg, $0.38 \mathrm{mmol}), \mathrm{HBr}(5 \mathrm{~mL}, 48 \%$ aq. solution) and acetic acid glacial ( $1 \mathrm{~mL})$ were stirred at $80{ }^{\circ} \mathrm{C}$ for $3.5 \mathrm{~h}$ (TLC monitored). After cooling to room temperature deionised water 
$(15 \mathrm{~mL})$ was added and the reaction neutralized using $\mathrm{NaHCO}_{(\mathrm{s})}$. The mixture was extracted with $\operatorname{DCM}(2 \times 100 \mathrm{~mL})$ and washed with deionised water $(1 \times 100 \mathrm{~mL})$. The organic layer was dried over $\mathrm{MgSO}_{4}$ and evaporated to dryness. After passing through a silica plug 37 was isolated as a cream colored solid (73 mg, 77\%): $\mathrm{mp} 258.3-261.8{ }^{\circ} \mathrm{C} ;{ }^{1} \mathrm{H}$ NMR (400 MHz, DMSO-d 6 ) $\delta 13.56(1 \mathrm{H}$, s), $8.39(2 \mathrm{H}, \mathrm{s}), 9.25(1 \mathrm{H}, \mathrm{s}), 8.46(1 \mathrm{H}, \mathrm{s}), 8.01(2 \mathrm{H}, \mathrm{m}), 7.50(3 \mathrm{H}, \mathrm{m}) ;{ }^{13} \mathrm{C}$ NMR $(100 \mathrm{MHz}, \mathrm{DMSO}-$ $\left.\mathrm{d}_{6}\right) \delta 159.2,158.3,156.4,144.5,134.6,133.2,129.3,128.8,128.5,127.8,125.9 ;$ MS (EI) $\mathrm{m} / \mathrm{z} 250.3$ $\left(\mathrm{M}^{+}, 100 \%\right)$; HRMS (EI) calcd for $\mathrm{C}_{14} \mathrm{H}_{10} \mathrm{~N}_{4} \mathrm{O} 250.0855$, found 250.0855 .

\section{2-Benzyl-6-phenyl-4-(pyridin-2-yl)pyridazin-3(2H)-one (38)}

A mixture of 36 (46 mg, $0.18 \mathrm{mmol})$, benzyl bromide $(0.02 \mathrm{~mL}, 0.19 \mathrm{mmol})$, potassium carbonate (64 mg, $0.46 \mathrm{mmol})$ and tetrabutylammonium bromide $(2.9 \mathrm{mg}, 0.009 \mathrm{mmol})$ in acetonitrile $(10 \mathrm{~mL})$ was heated at reflux for $1 \mathrm{~h}$. The solvent was then removed in vacuo before column chromatography on silica, eluent EtOAc to yield 38 as yellow needles after recrystallization from hexane (58 $\mathrm{mg}$, 93\%): mp 157.1-159.4 ${ }^{\circ} \mathrm{C} ;{ }^{1} \mathrm{H}$ NMR (400 MHz, $\left.\mathrm{CDCl}_{3}\right) \delta 8.73(3 \mathrm{H}, \mathrm{m}), 7.95(2 \mathrm{H}, \mathrm{d}, J=6.8 \mathrm{~Hz})$, $7.81(1 \mathrm{H}, \mathrm{t}, J=8.8 \mathrm{~Hz}), 7.55(2 \mathrm{H}, \mathrm{d}, J=6.8 \mathrm{~Hz}), 7.47(3 \mathrm{H}, \mathrm{m}), 7.32(4 \mathrm{H}, \mathrm{m}), 5.54(2 \mathrm{H}, \mathrm{s}) ;{ }^{13} \mathrm{C} \mathrm{NMR}$ $\left(100 \mathrm{MHz} \mathrm{CDCl}_{3}\right) \delta 159.4,151.2,149.7,145.0,136.8,136.7,136.6,135.3,129.5,129.01,128.97$,

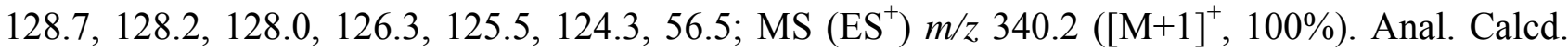
for $\mathrm{C}_{22} \mathrm{H}_{17} \mathrm{~N}_{3} \mathrm{O}$ : C, 77.86; H, 5.05; N, 12.38. Found: C, 77.42; H, 4.98; N, 12.29\%. Crystals for X-ray structure determination were grown from hexane.

\section{Pyridinium salt (39)}

To a methanol solution of compound $36(30 \mathrm{mg}), \mathrm{HBF}_{4}$ solution in diethyl ether (Fluka, 54\%) was added slowly until precipitation was complete. The solids were redissolved by heating the mixture. Slow evaporation of the cooled solution gave orange crystals of $\mathbf{3 9}$ suitable for X-ray structural analysis.

\section{$\mathbf{1} \cdot \mathbf{B}(\mathrm{OH})_{3}$ Complex}

3,6-Dimethoxy-4-pyridazinylboronic acid 2 was refluxed in the minimal amount of ethanol, upon cooling and evaporation of the solvent crystals grew which X-ray structural analysis confirmed to be complex $\mathbf{1} \cdot \mathbf{B}(\mathbf{O H})_{3}$. 


\section{X-Ray Crystallography}

Single-crystal diffraction experiments were carried out on Bruker 3-circle diffractometers with SMART 1K CCD area detector, using graphite-monochromated Mo- $K_{\alpha}$ radiation $(\lambda=0.71073 \AA)$ and Cryostream (Oxford Cryosystems) open-flow $\mathrm{N}_{2}$ cryostats. The structures were solved by direct methods and refined by full-matrix least squares against $F^{2}$ of all data, using SHELXTL software. ${ }^{7}$ Non-hydrogen atoms were refined in anisotropic, $\mathrm{H}$ atoms is isotropic approximation or (in 20b and 20d) in "riding" model. Crystal data and other experimental details are listed in Table S1. Full structural data (excluding structure factors) have been deposited at the Cambridge Crystallographic Data Centre.

The crystal of $\mathbf{1} \cdot \mathrm{B}(\mathrm{OH})_{3}$ comprises discrete neutral molecules of $\mathbf{1}$ and orthoboric acid (both planar and forming a dihedral angle of $7^{\circ}$ ), hydrogen-bonded into a centrosymmetric $\left[\mathbf{1} \cdot \mathrm{B}(\mathrm{OH})_{3}\right]_{2}$ unit (Fig. S1). The $\mathrm{O}(4)$ and $\mathrm{O}(5)$ act as donor of hydrogen bonds, whereas $\mathrm{O}(3)$ is both donor and acceptor; the B-O(3) bond is longer (1.378(2) $\AA$ ) than B-O(4) and B-O(5) (1.359(2) and 1.361(2) $\AA$ ). In sassolite (pure $\mathrm{B}(\mathrm{OH})_{3}$ ) every oxygen atom in two independent molecules is both donor and acceptor; all B-O distances are equivalent within experimental error, but the average (1.368(1) at 105 $\mathrm{K})^{8}$ is essentially the same as in $\mathbf{1} \cdot \mathrm{B}(\mathrm{OH})_{3}$.

In molecule 20b (Fig. S2) the dihedral angle between rings $i$ and ii is $22.0^{\circ}$, between ring $i$ and the boron atom plane is $28.0^{\circ}$; torsion angles $\mathrm{C}(7)-\mathrm{O}(1)-\mathrm{C}(3)-\mathrm{N}(2) 8.5(2)^{\circ}, \mathrm{C}(8)-\mathrm{O}(2)-\mathrm{C}(24)-$ $\mathrm{C}(23) 15.6(2)^{\circ}$. Ring iii adopts twisted conformation (ca. $C_{2}$ symmetry).

In molecule 20d (Fig. S3) the tetramethyldioxaborolan moiety is disordered between two different conformations with equal probability. The pyridazine ring forms dihedral angles of $21.8^{\circ}$ with the pyridine ring and $21.7^{\circ}$ with the $\mathrm{B}$ atom plane. The methoxy group has an in-plane conformation (torsion angle $\left.\mathrm{N}(12)-\mathrm{C}(13)-\mathrm{O}(3)-\mathrm{C}(7) 4.6(2)^{\circ}\right)$.

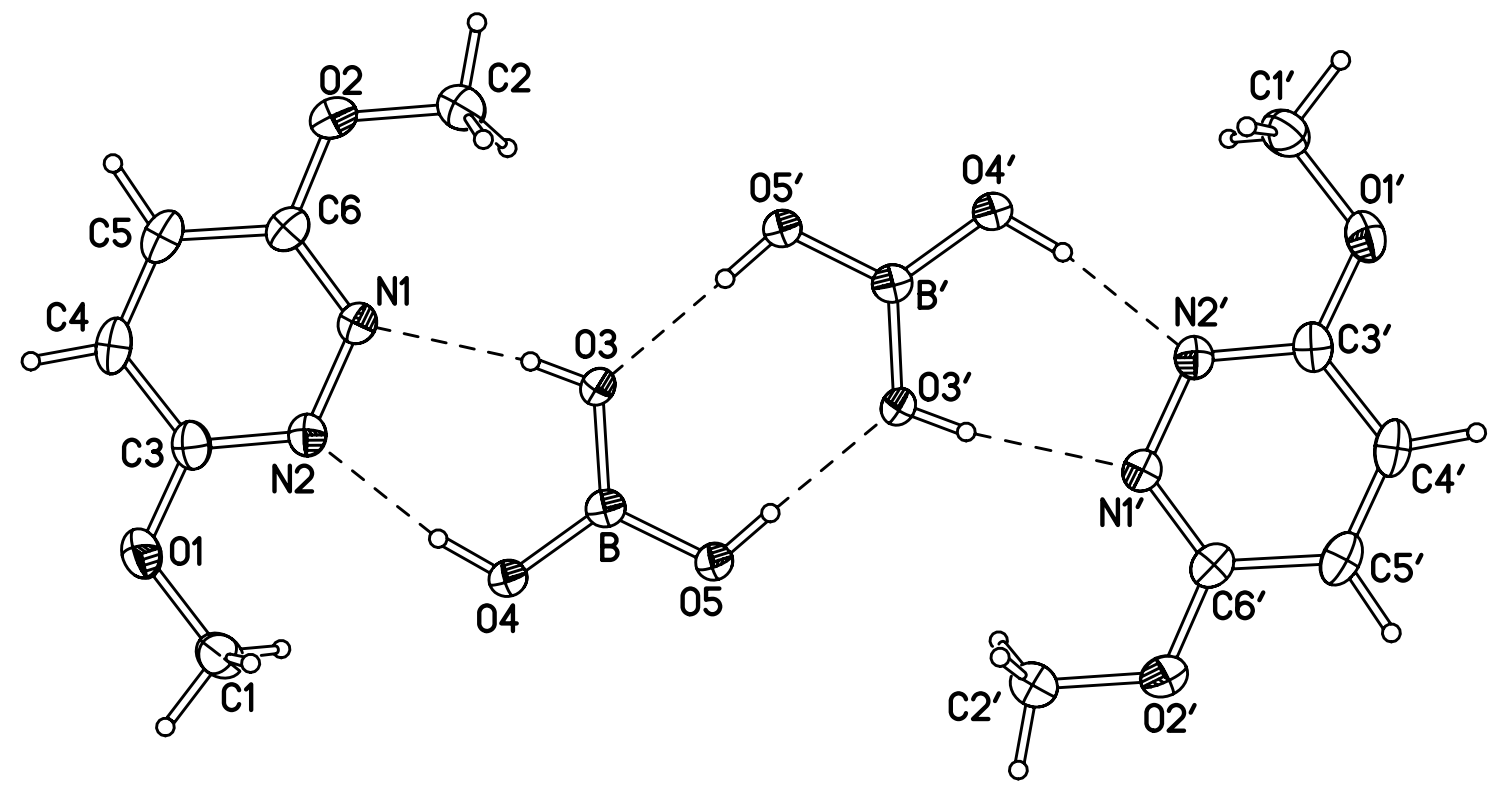

Figure S1. X-ray structure of $1 \cdot \mathrm{B}(\mathrm{OH})_{3}$. Henceforth thermal ellipsoids are drawn at the $50 \%$ probability level. Primed atoms are generated by the crystallographic inversion center. 


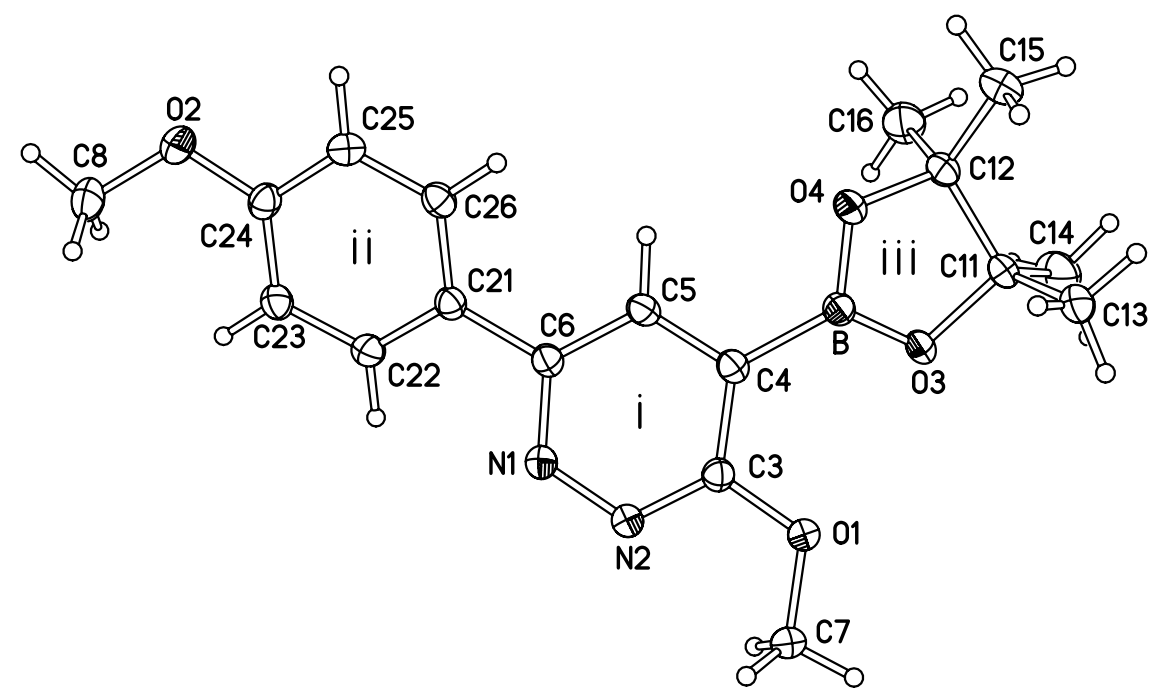

Figure S2. X-ray structure of $20 \mathrm{~b}$ (50\% thermal ellipsoids).

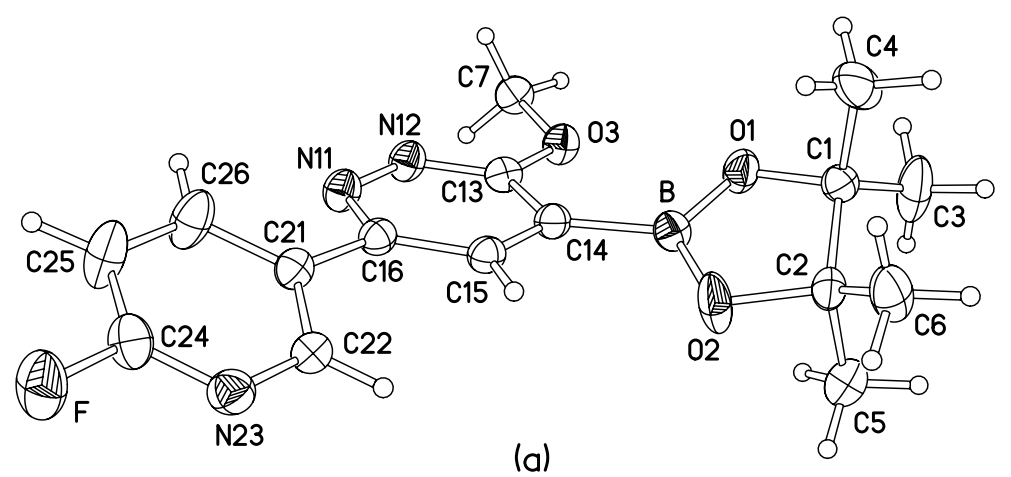

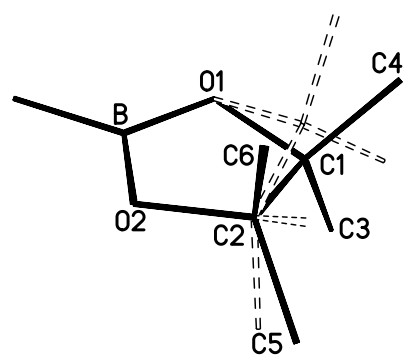

(b)

Figure S3. X-ray structure of 20d: (a) major conformation; (b) disorder of the tetramethyldioxaborolan moiety.

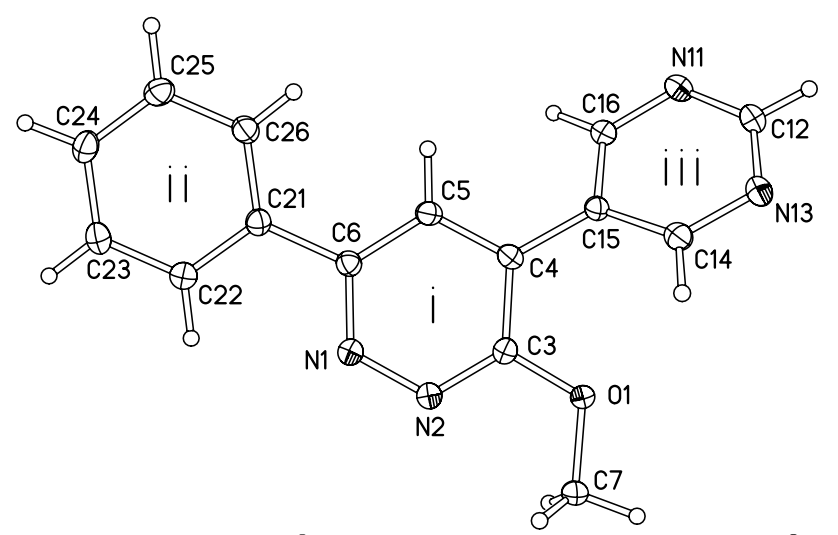

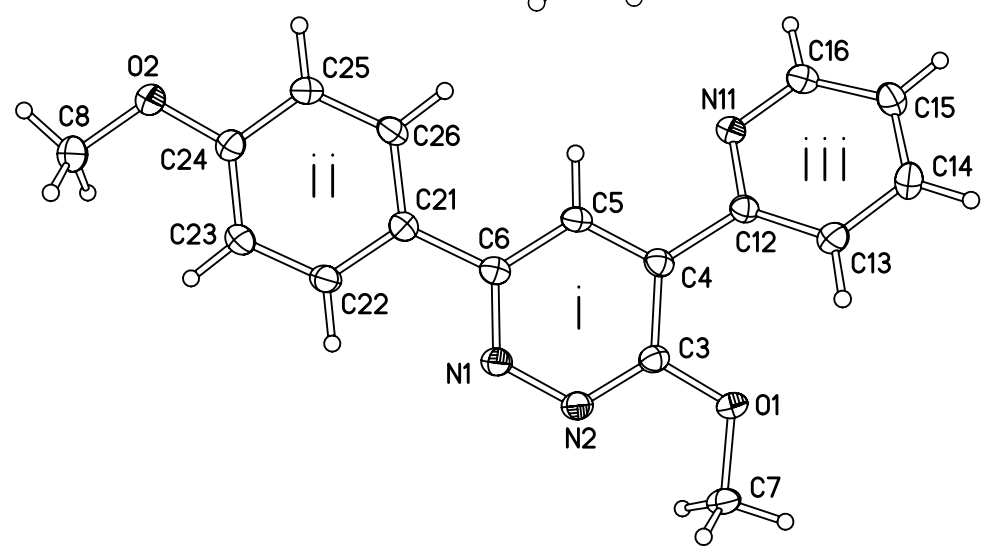

Figure S4. X-ray structures of 22 (top) and 24. 
In molecule 22 rings $i$ and $i$ form a dihedral angle of $8.2^{\circ}$, rings $i$ and iii of $37.2^{\circ}$, in 24 the corresponding angles are similar (i/ii $7.8^{\circ}$, i/iii $\left.31.8^{\circ}\right)$. The methoxy groups are coplanar with the adjacent rings: rigorously in 22, nearly in $\mathbf{2 4}$ where the torsion angles $\mathrm{N}(2)-\mathrm{C}(3)-\mathrm{O}(1)-\mathrm{C}(7)$ and $\mathrm{C}(23)-\mathrm{C}(24)-\mathrm{O}(2)-\mathrm{C}(8)$ equal $3.5(2)$ and $2.0(2)^{\circ}$, respectively.
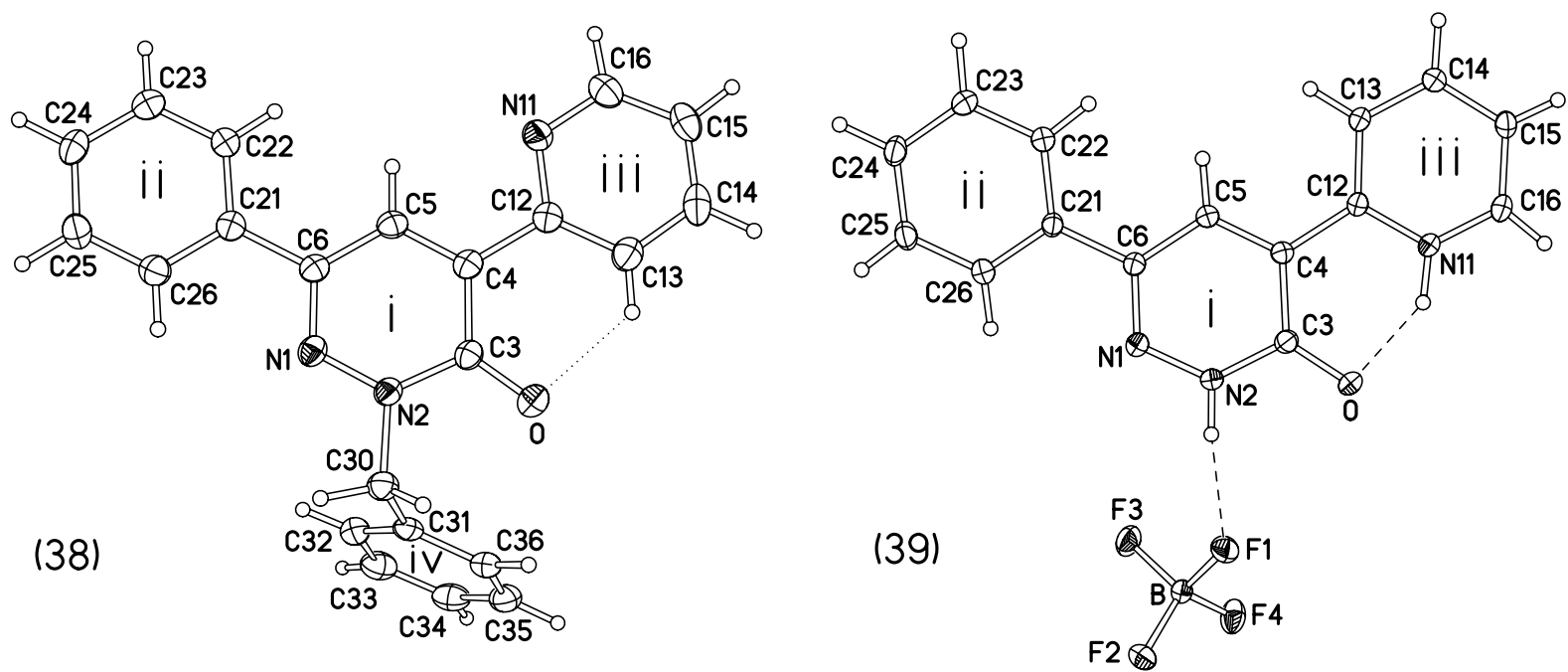

Figure S5. X-ray molecular structures of 38 and 39 (50\% thermal ellipsoids). Angles between ring planes $\left({ }^{\circ}\right)$ : $\mathrm{i} /$ ii 5.2 , i/iii 1.3, i/iv 75.8 in 38, i/ii 12.7, i/iii 2.6 in 39. Note the much smaller atomic displacements in 39 at the same temperature $(120 \mathrm{~K})$.

X-ray diffraction studies of 38 and 39 (Fig. S5) revealed that in both cases rings $i$ - iii are nearly coplanar, but the orientation of ring iii differs by a ca. $180^{\circ}$ rotation. Thus a weak intramolecular CH...O hydrogen bond of 38 (C-H 0.94(2), H...O 2.15(2), C(3)-O 1.238(2) A) is replaced by a strong N-H...O bond in 39 (N-H 0.95(2), H...O 1.80(2), C(3)-O 1.2474(13) $\AA$ ). Comparison of the C(4)$\mathrm{C}(12)-\mathrm{N}(11)$ and $\mathrm{C}(4)-\mathrm{C}(12)-\mathrm{C}(13)$ angles $\left(115.08(15)\right.$ and $123.69(16)^{\circ}$ in 38, 117.92(9) and $124.12(9)^{\circ}$ in $\mathbf{3 9}$, respectively) indicates a pyridyl...O repulsion in $\mathbf{3 8}$ and attraction in $\mathbf{3 9}$. The crystal structure of 39 comprises cation-anion pairs, linked by an N-H...F hydrogen bond. 
Table S1. Crystal data $(T=120 \mathrm{~K})$

\begin{tabular}{|c|c|c|c|c|c|c|c|}
\hline Compound & 20b & 20d & 22 & 24 & 38 & 39 & $\mathbf{1} \cdot \mathrm{B}(\mathrm{OH})_{3}$ \\
\hline CCDC dep. No. & 665168 & 665169 & 665170 & 665171 & 665172 & 665173 & 665174 \\
\hline Empirical formula & $\mathrm{C}_{18} \mathrm{H}_{23} \mathrm{BN}_{2} \mathrm{O}_{4}$ & $\mathrm{C}_{16} \mathrm{H}_{19} \mathrm{BFN}_{3} \mathrm{O}_{3}$ & $\mathrm{C}_{15} \mathrm{H}_{12} \mathrm{~N}_{4} \mathrm{O}$ & $\mathrm{C}_{17} \mathrm{H}_{15} \mathrm{~N}_{3} \mathrm{O}_{2}$ & $\mathrm{C}_{22} \mathrm{H}_{17} \mathrm{~N}_{3} \mathrm{O}$ & $\mathrm{C}_{15} \mathrm{H}_{12} \mathrm{~N}_{3} \mathrm{O}^{+} \mathrm{BF}_{4}^{-}$ & $\mathrm{C}_{6} \mathrm{H}_{8} \mathrm{~N}_{2} \mathrm{O}_{2} \cdot \mathrm{H}_{3} \mathrm{BO}_{3}$ \\
\hline Crystal system & monoclinic & monoclinic & triclinic & monoclinic & monoclinic & triclinic & monoclinic \\
\hline Space group (No.) & $P 2_{1} / n(\# 14)$ & $P 2_{1} / n(\# 14)$ & $P-1(\# 2)$ & $P 2_{1} / n(14)$ & $P 2_{1} / n(14)$ & $P-1(\# 2)$ & $P 2_{1} / c(14)$ \\
\hline$b, \AA$ & $22.418(2)$ & $22.646(2)$ & $10.556(1)$ & $21.962(3)$ & $32.701(3)$ & $8.4696(11)$ & $18.250(2)$ \\
\hline$c, \AA$ & $11.2301(12)$ & $11.128(1)$ & $15.381(2)$ & $15.6265(16)$ & $10.766(1)$ & $10.4997(14)$ & $7.5773(9)$ \\
\hline$\alpha . \AA$ & 90 & 90 & $76.94(1)$ & 90 & 90 & $87.23(1)$ & 90 \\
\hline$\beta, \AA$ & $98.45(1)$ & $106.52(1)$ & $83.20(1)$ & $90.73(1)$ & $90.55(1)$ & $71.32(1)$ & $93.38(1)$ \\
\hline$\rho$ (calc.), $\mathrm{g} / \mathrm{cm}^{3}$ & 1.291 & 1.351 & 1.450 & 1.404 & 1.357 & 1.626 & 1.381 \\
\hline$\mu(\mathrm{Mo}-K \alpha), \mathrm{mm}^{-1}$ & 0.09 & 0.10 & 0.10 & 0.10 & 0.09 & 0.14 & 0.12 \\
\hline Reflections collected & 17656 & 19148 & 7255 & 13751 & 16257 & 8243 & 8218 \\
\hline Independent reflections & 4678 & 4313 & 3174 & 3691 & 3781 & 3623 & 2572 \\
\hline$R($ int $)$ & 0.032 & 0.045 & 0.024 & 0.032 & 0.039 & 0.035 & 0.038 \\
\hline$R(F)[I>2 \sigma(I)]$ & 0.043 & 0.045 & 0.041 & 0.042 & 0.054 & 0.036 & 0.047 \\
\hline$w R\left(F^{2}\right)$ [all data] & 0.109 & 0.115 & 0.111 & 0.109 & 0.125 & 0.106 & 0.113 \\
\hline
\end{tabular}


3,6-Dimethoxy-4-pyridazinylboronic acid (2)

${ }^{1}$ H NMR (400 MHz, DMSO-d $_{6}$ )

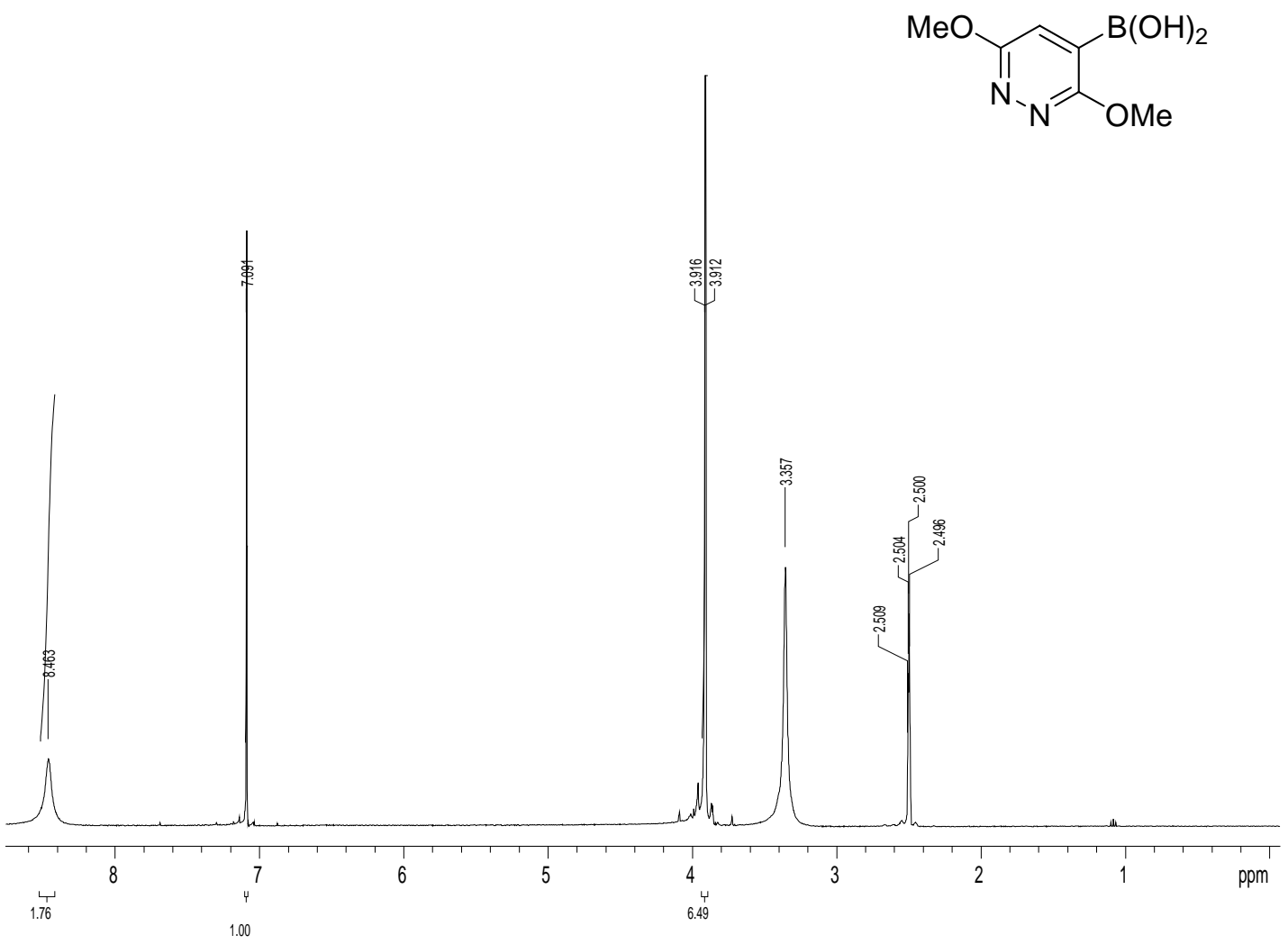

${ }^{13} \mathrm{C}$ NMR (100 MHz, DMSO-d 6 )

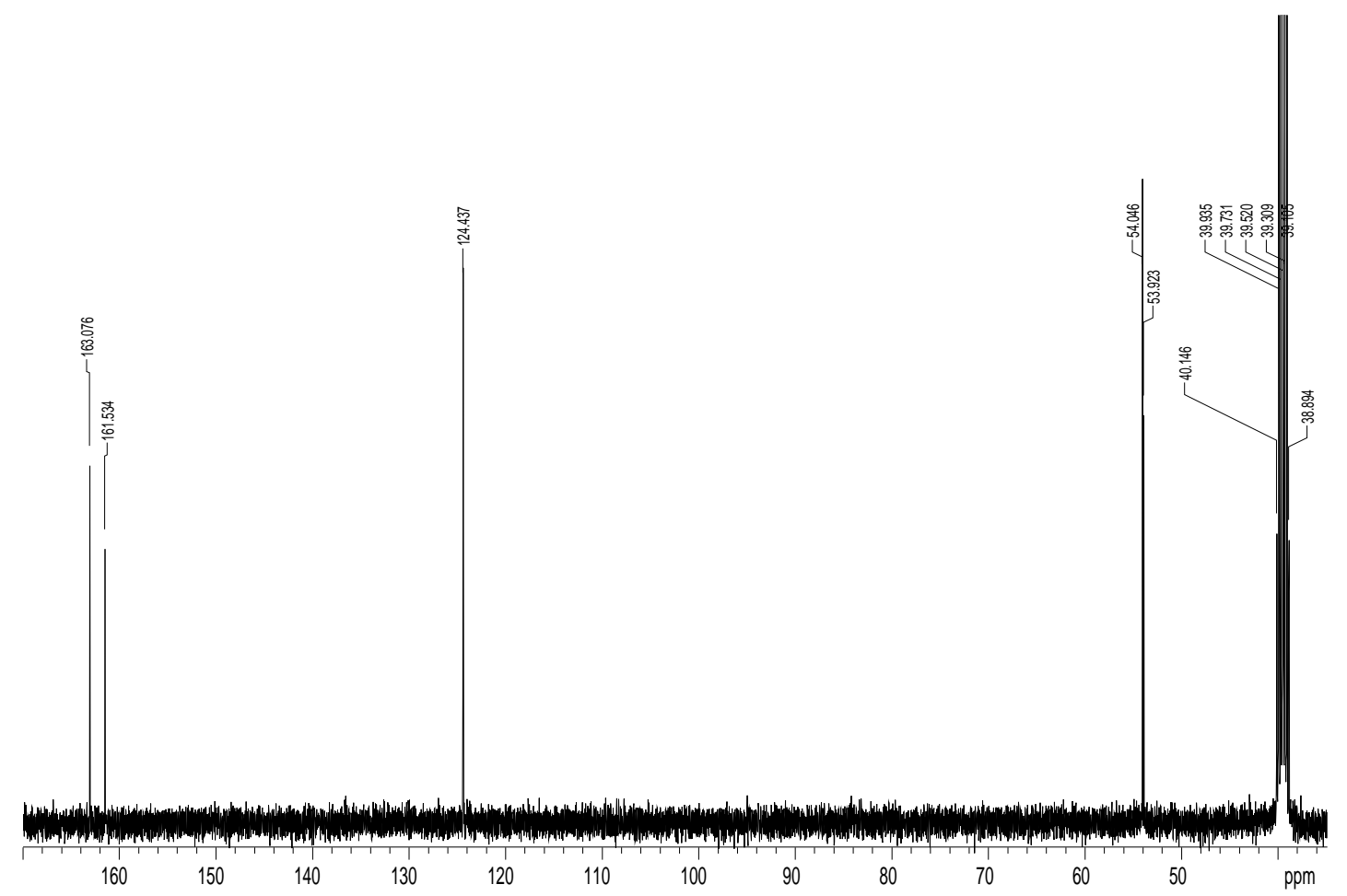


2-(3,6-Dimethoxypyridazin-4-yl)-5-nitropyridine (10)

${ }^{1} \mathrm{H}$ NMR $\left(400 \mathrm{MHz}, \mathrm{CDCl}_{3}\right)$

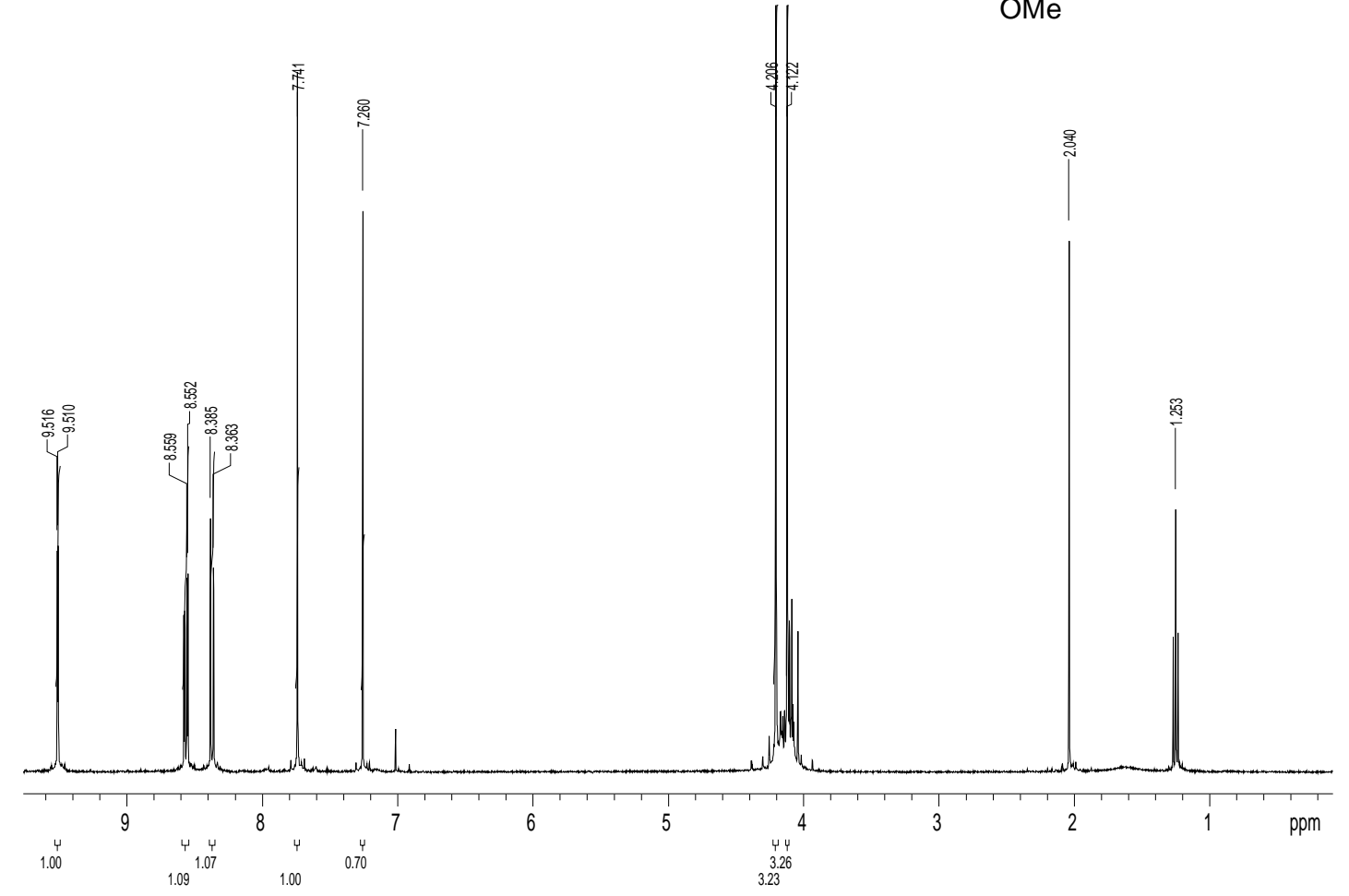

${ }^{13} \mathrm{C}$ NMR (100 MHz, $\left.\mathrm{CDCl}_{3}\right)$

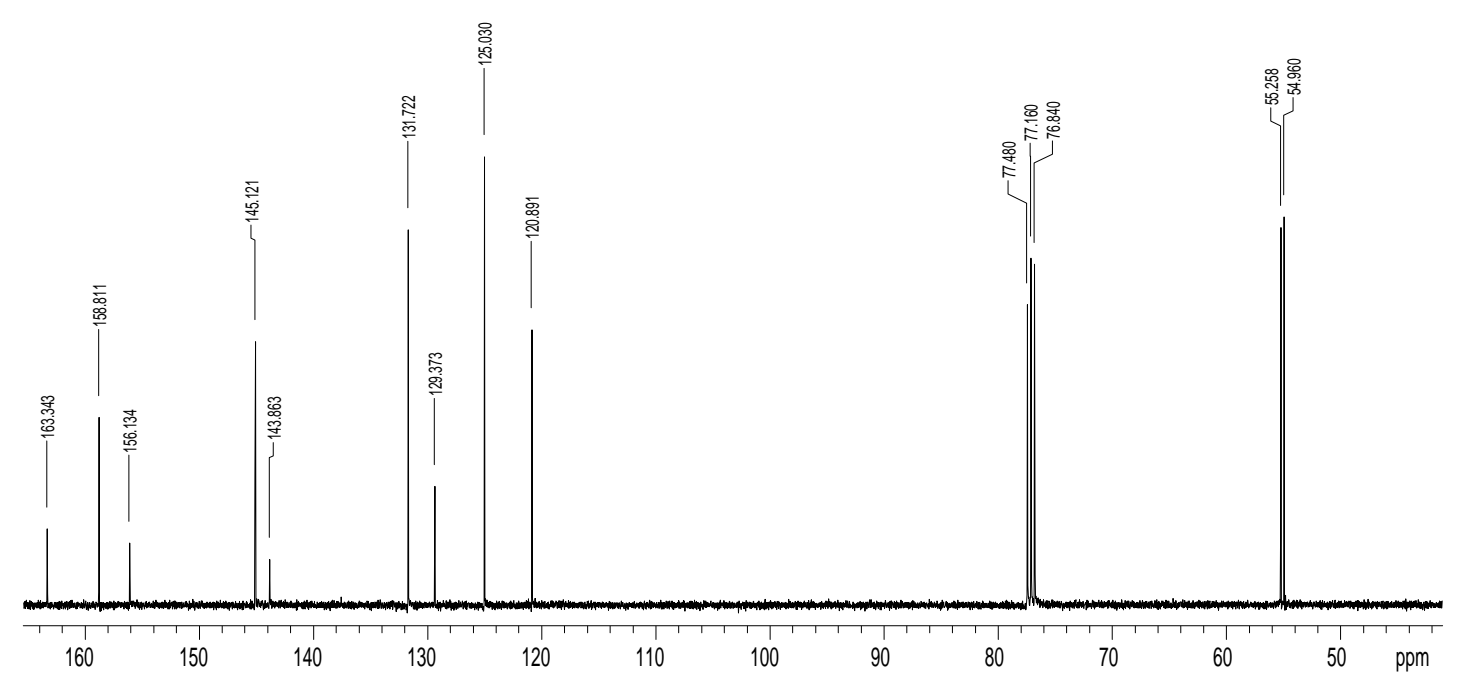


2-Amino-3-nitro-5-(3,6-dimethoxypyridazin-4-yl)-pyridine (11)

${ }^{1} \mathrm{H}$ NMR (400 MHz, DMSO-d 6 )

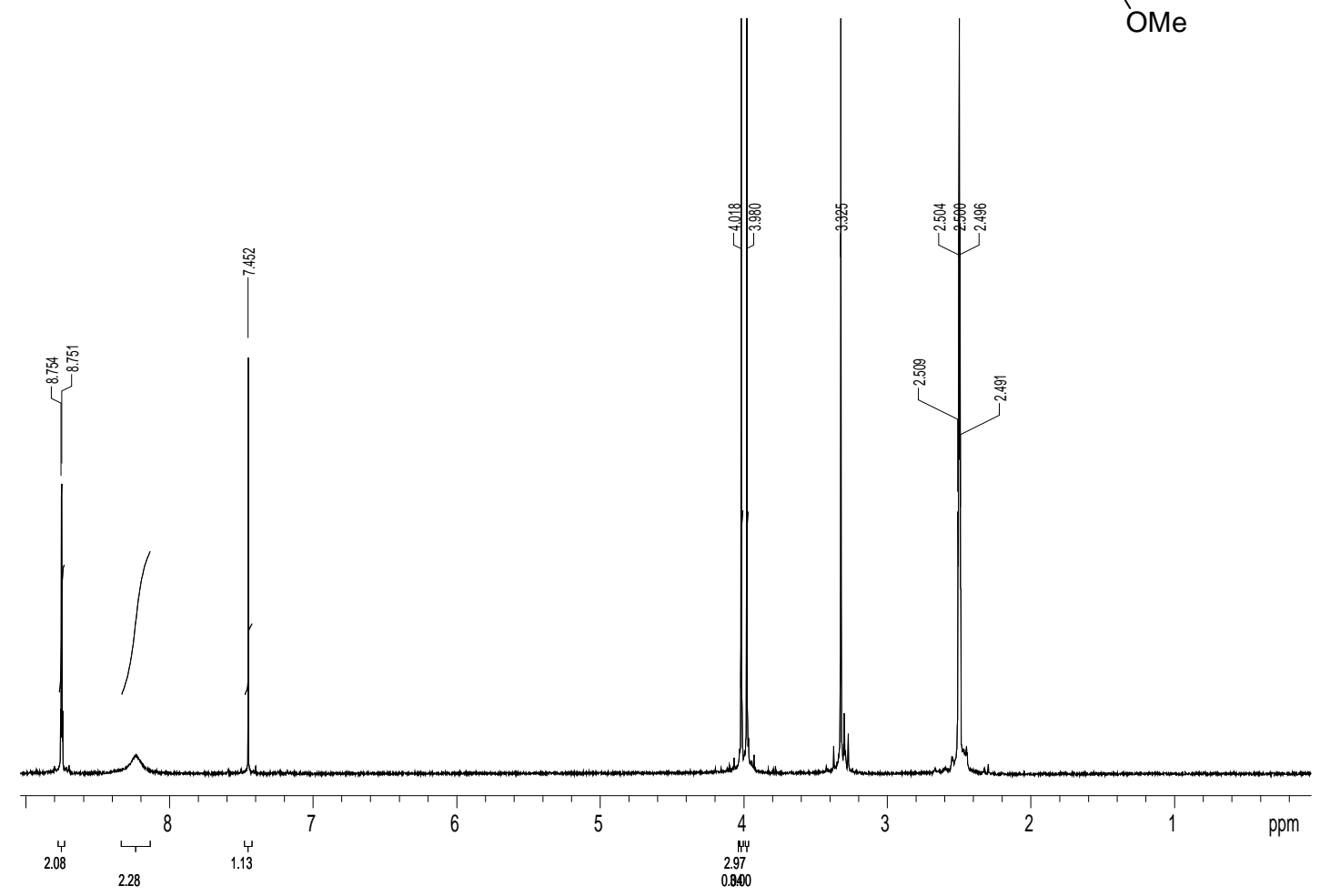

${ }^{13} \mathrm{C}$ NMR (100 MHz, DMSO-d 6 )

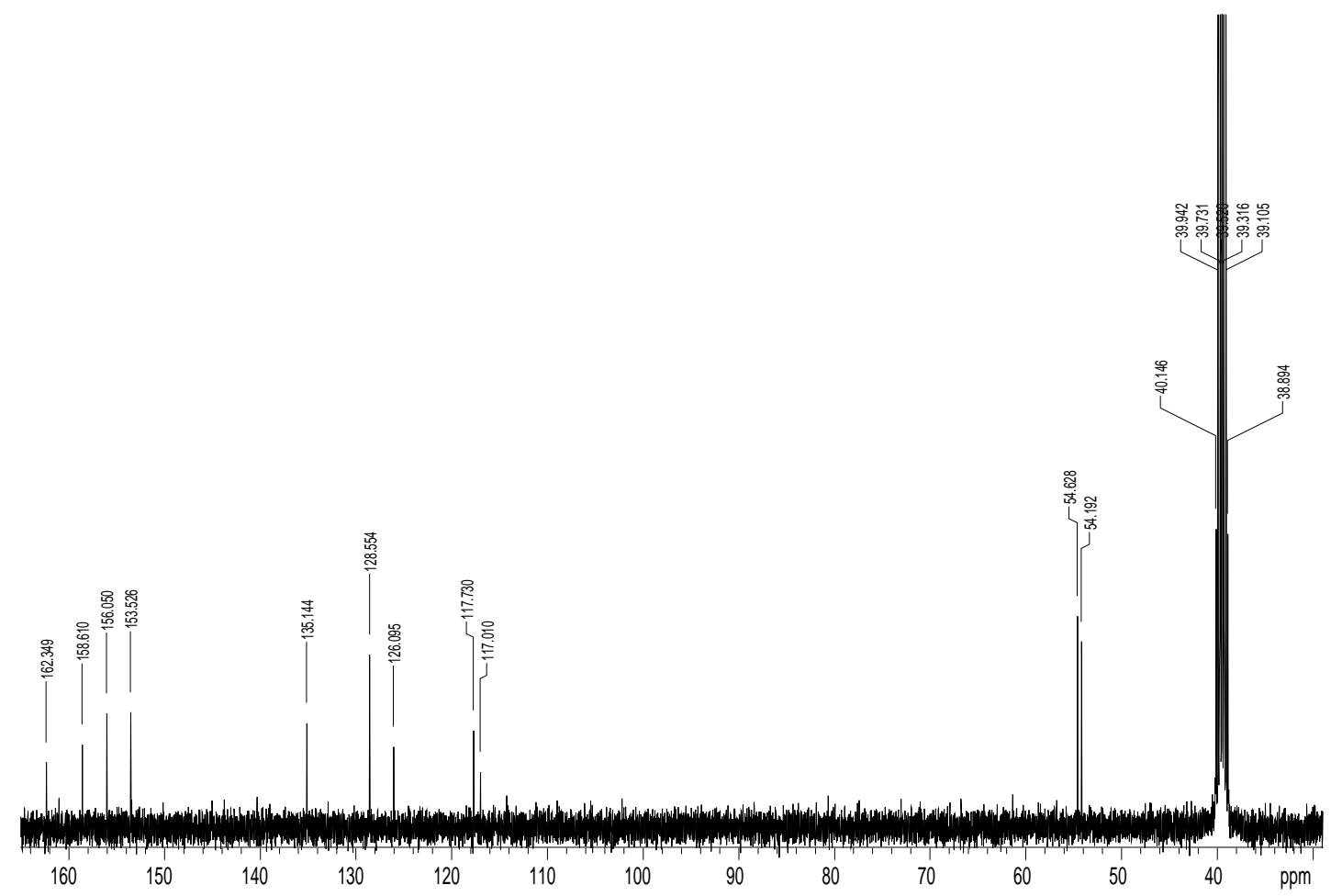


5-(3,6-Dimethoxypyridazin-4-yl)-pyrimidine (12)

${ }^{1} \mathrm{H}$ NMR (400 MHz, $\mathrm{CDCl}_{3}$ )

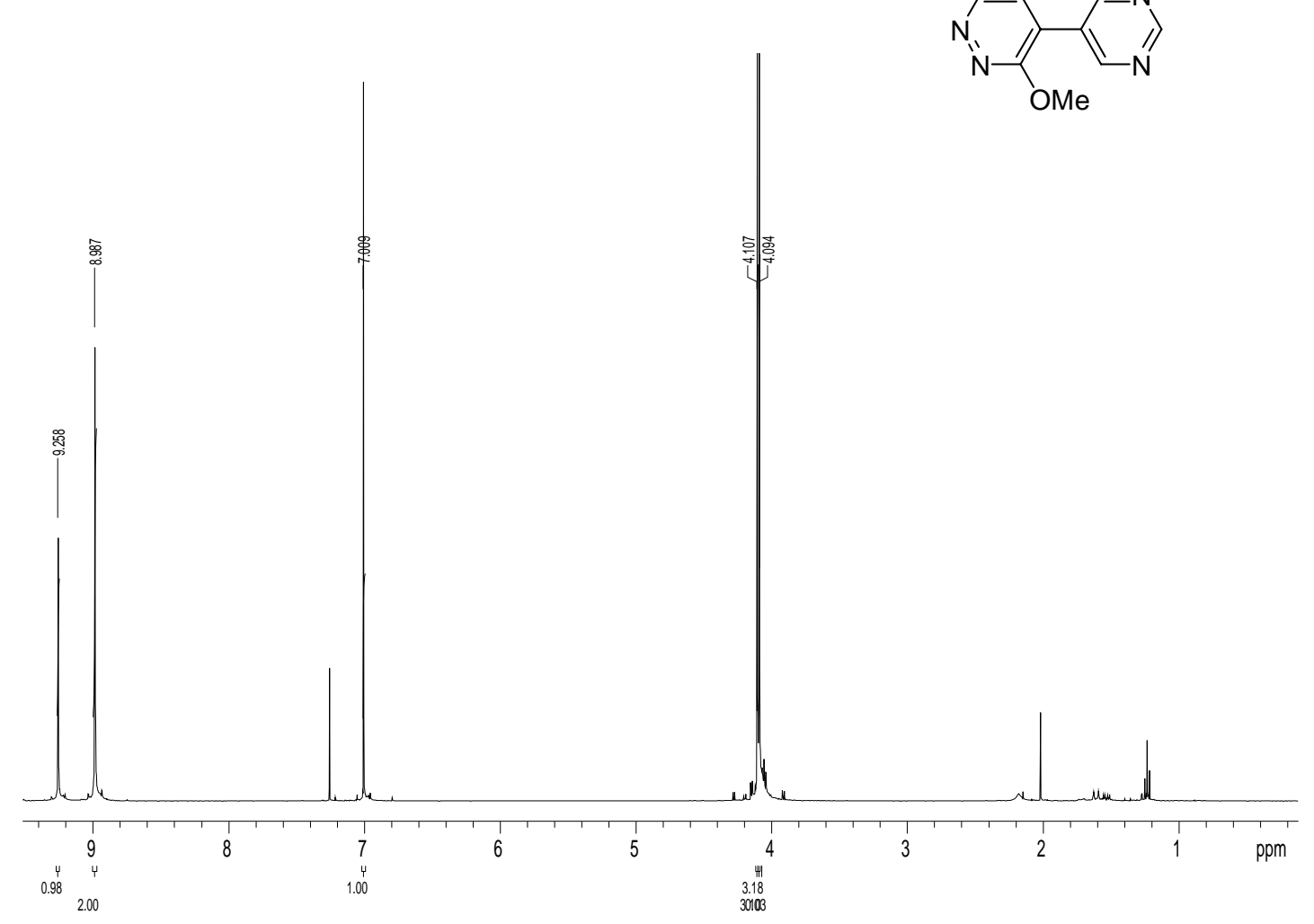

${ }^{13} \mathrm{C}$ NMR (100 MHz, $\left.\mathrm{CDCl}_{3}\right)$

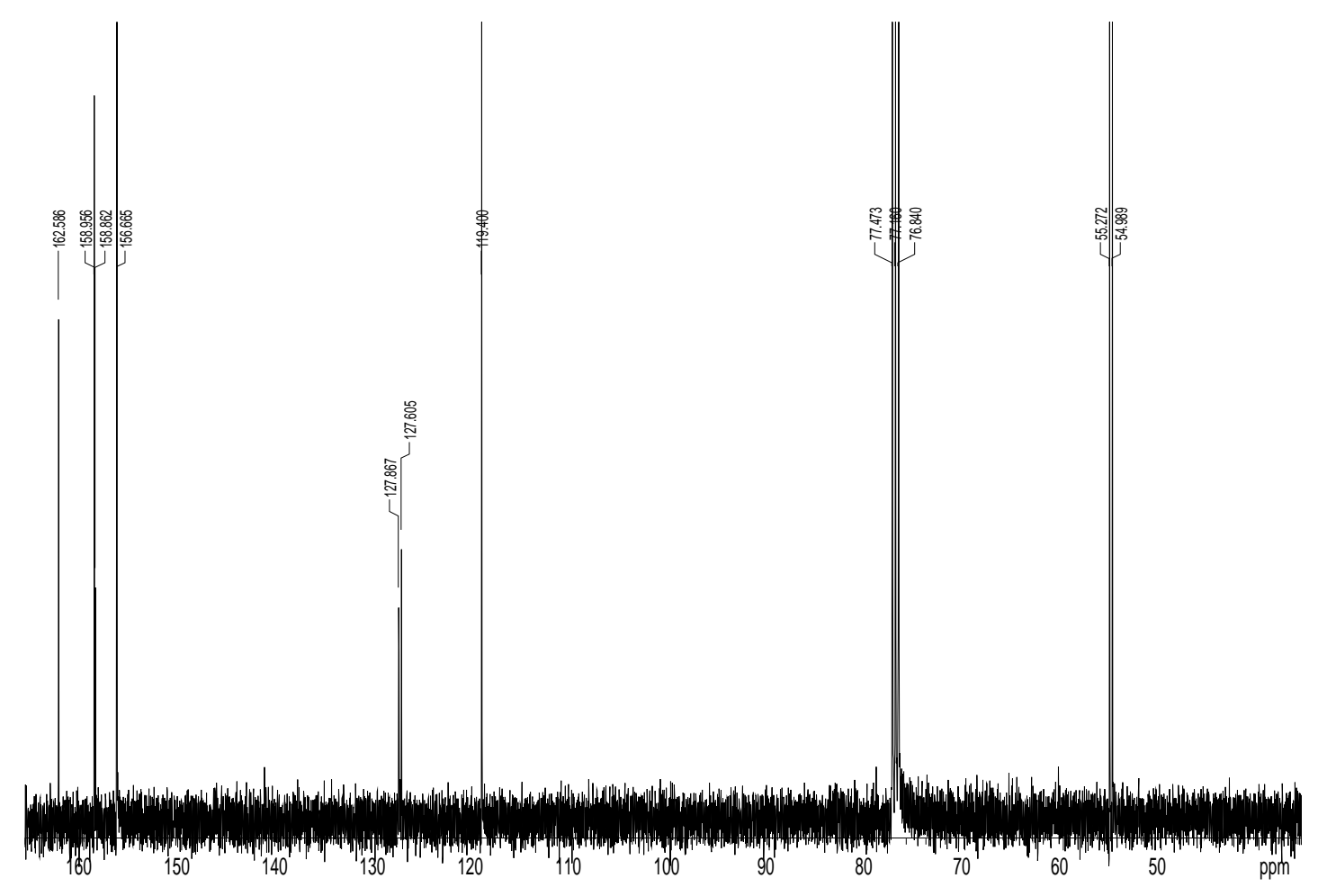


2-Amino-5-(3,6-dimethoxypyridazin-4-yl)-pyrimidine (13)

${ }^{1} \mathrm{H}$ NMR (400 MHz, $\mathrm{CDCl}_{3}$ )

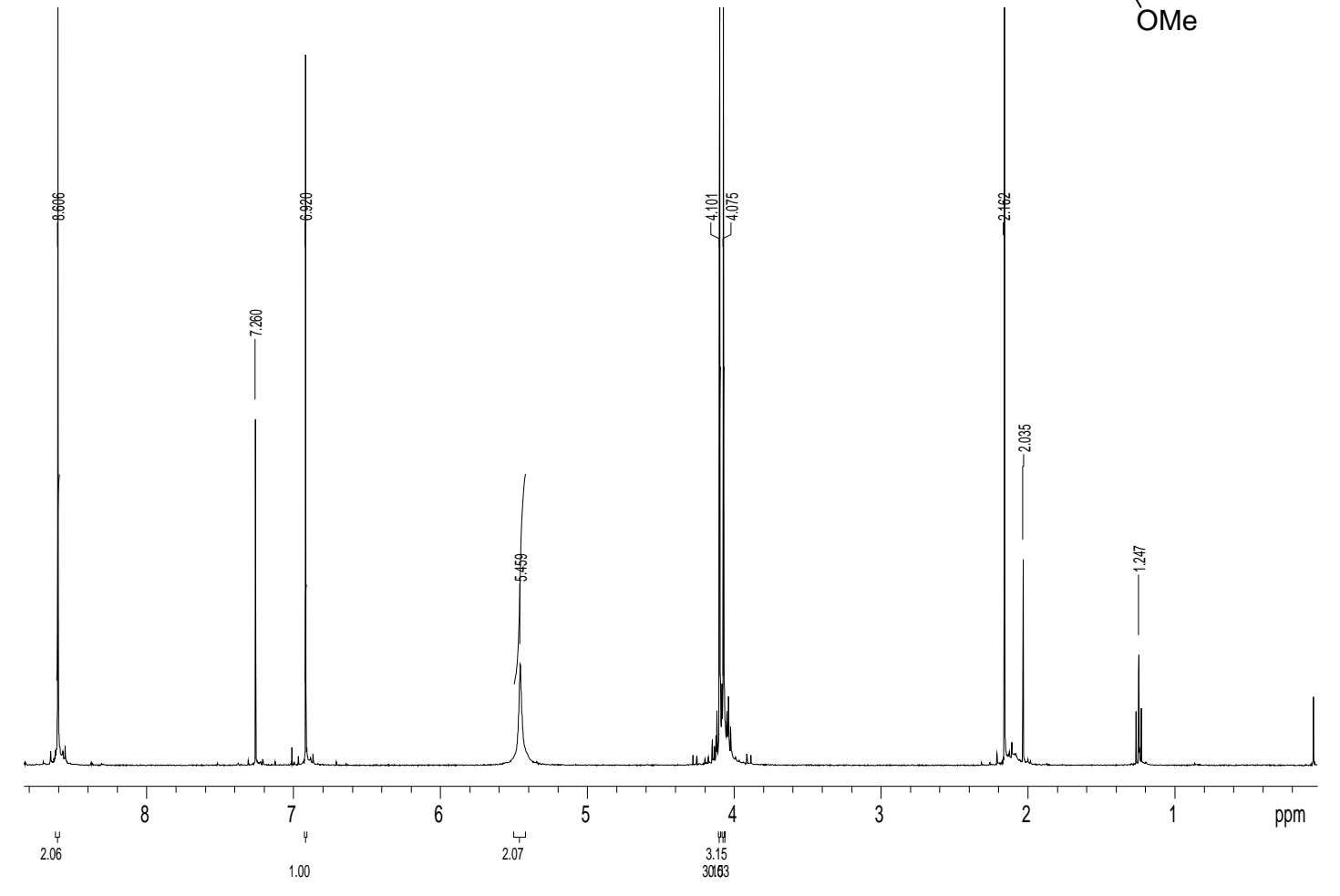

${ }^{13} \mathrm{C}$ NMR (100 MHz, $\mathrm{CDCl}_{3}$ )

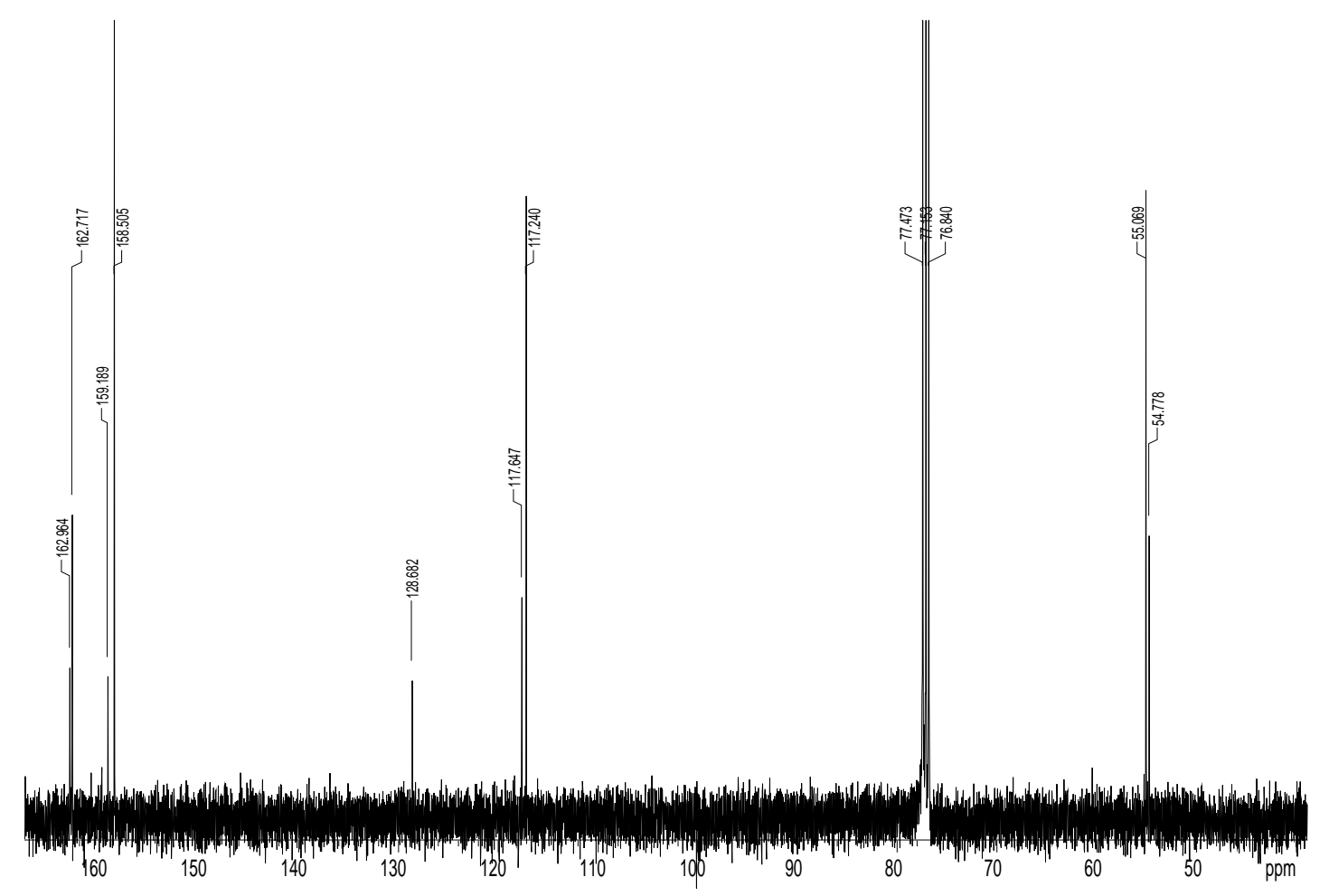


2-Amino-5-(3,6-dimethoxypyridazin-4-yl)-pyrazine (14)

${ }^{1} \mathrm{H}$ NMR (400 MHz, DMSO-d )

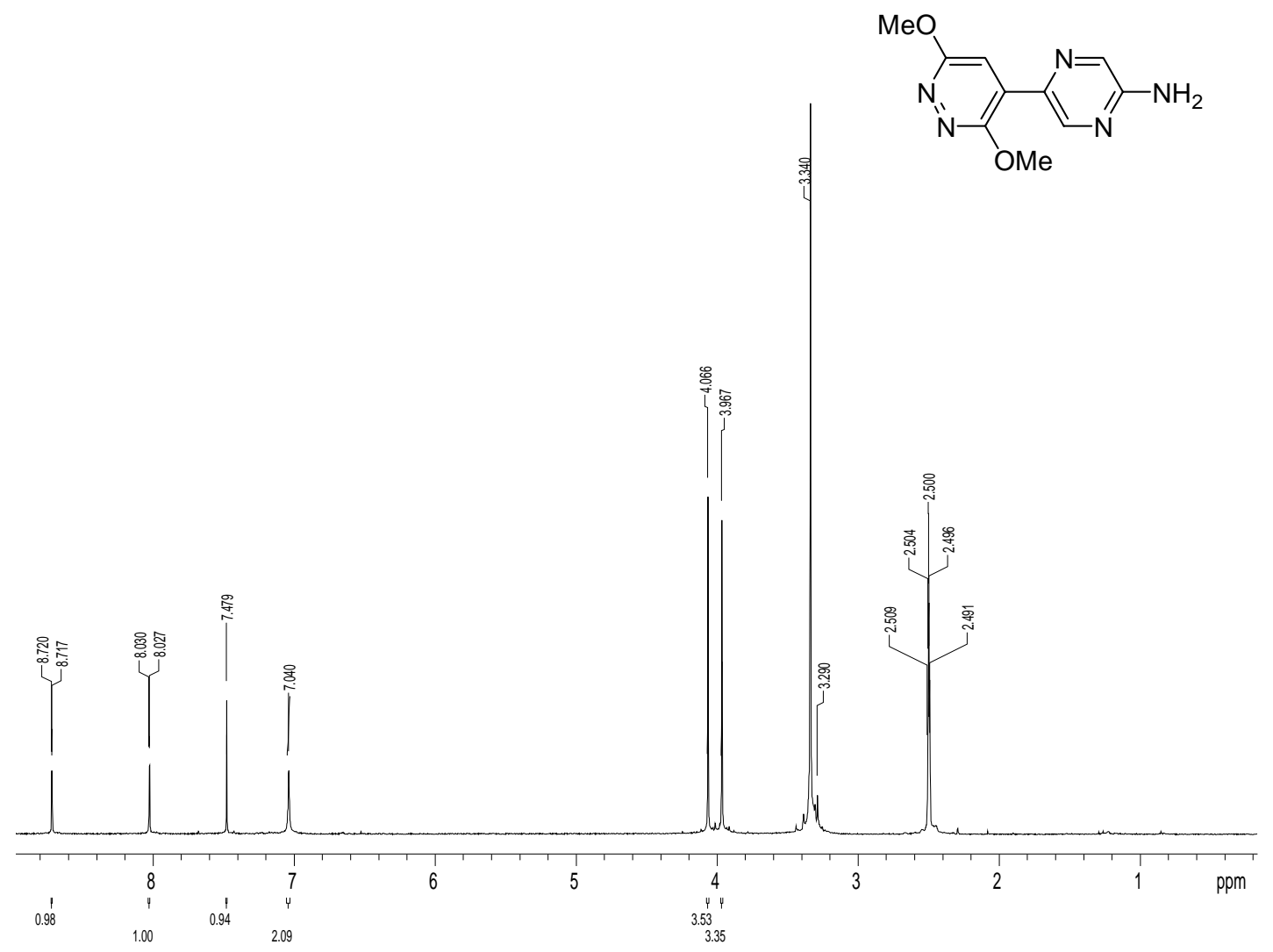

${ }^{13} \mathrm{C}$ NMR (100 MHz, DMSO-d 6 )

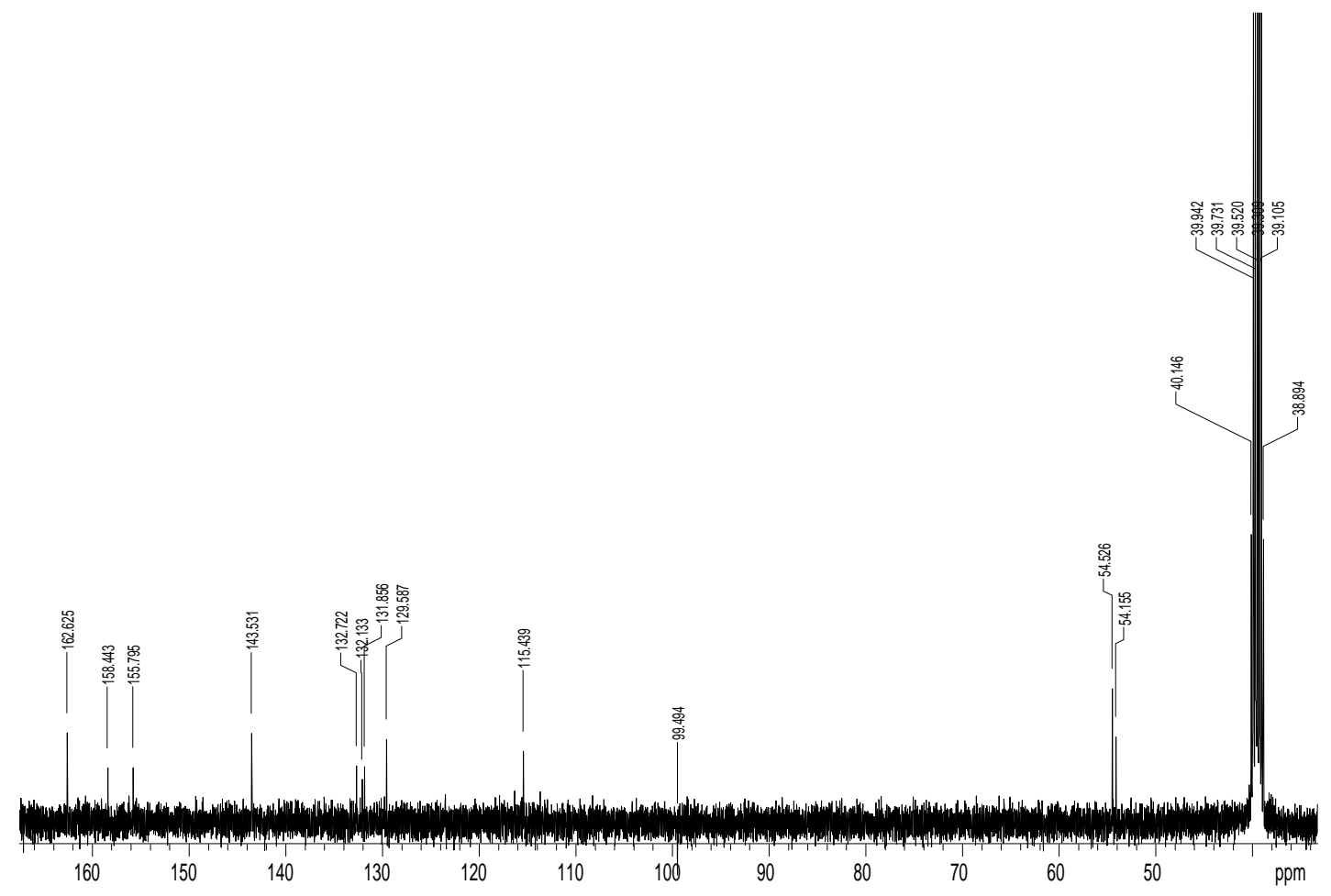


2-Nitro-5-(3,6-dimethoxypyridazin-4-yl)-thiophene (15)

${ }^{1} \mathrm{H}$ NMR (400 MHz, $\mathrm{CDCl}_{3}$ )

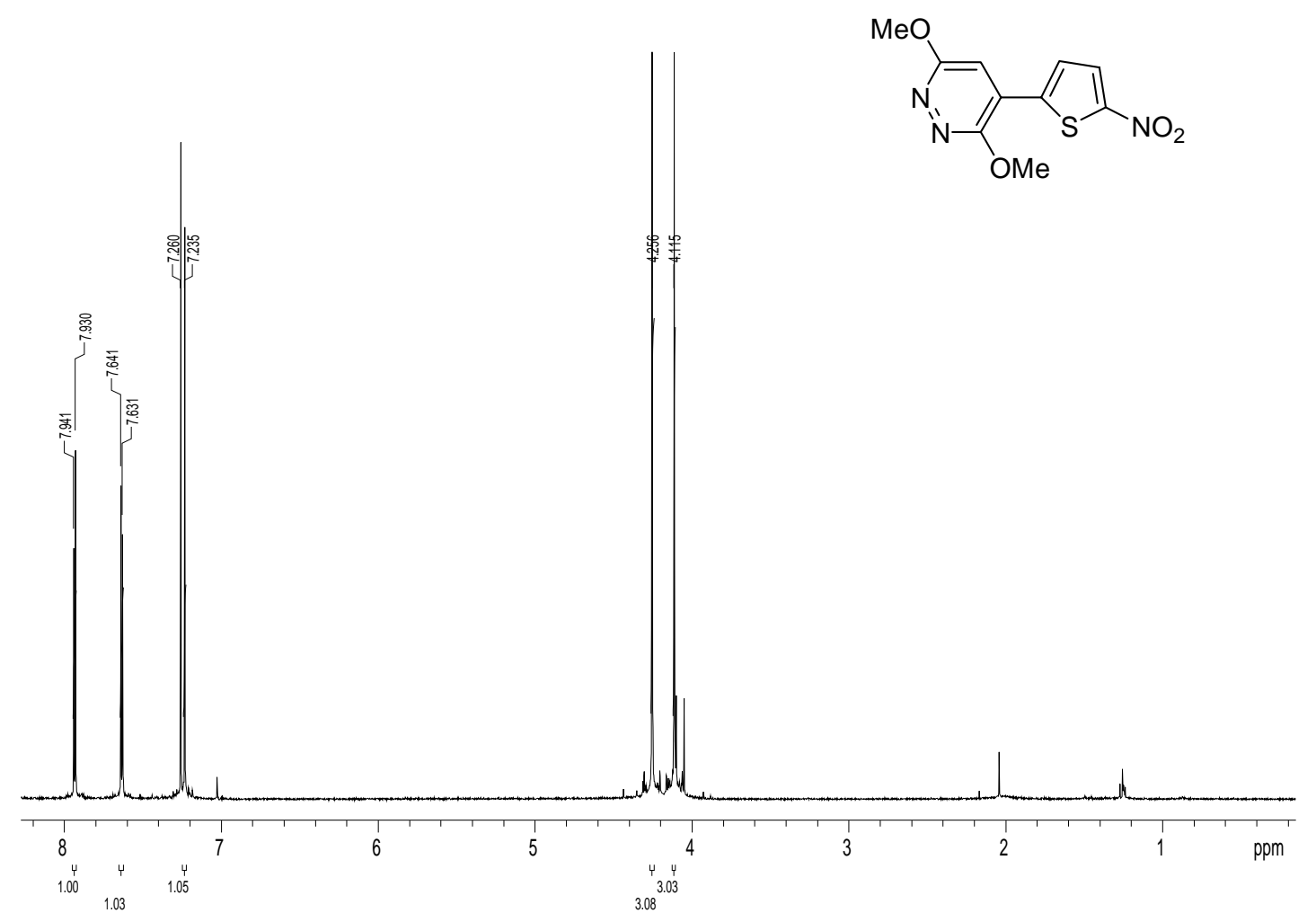

${ }^{13} \mathrm{C}$ NMR (100 MHz, $\left.\mathrm{CDCl}_{3}\right)$

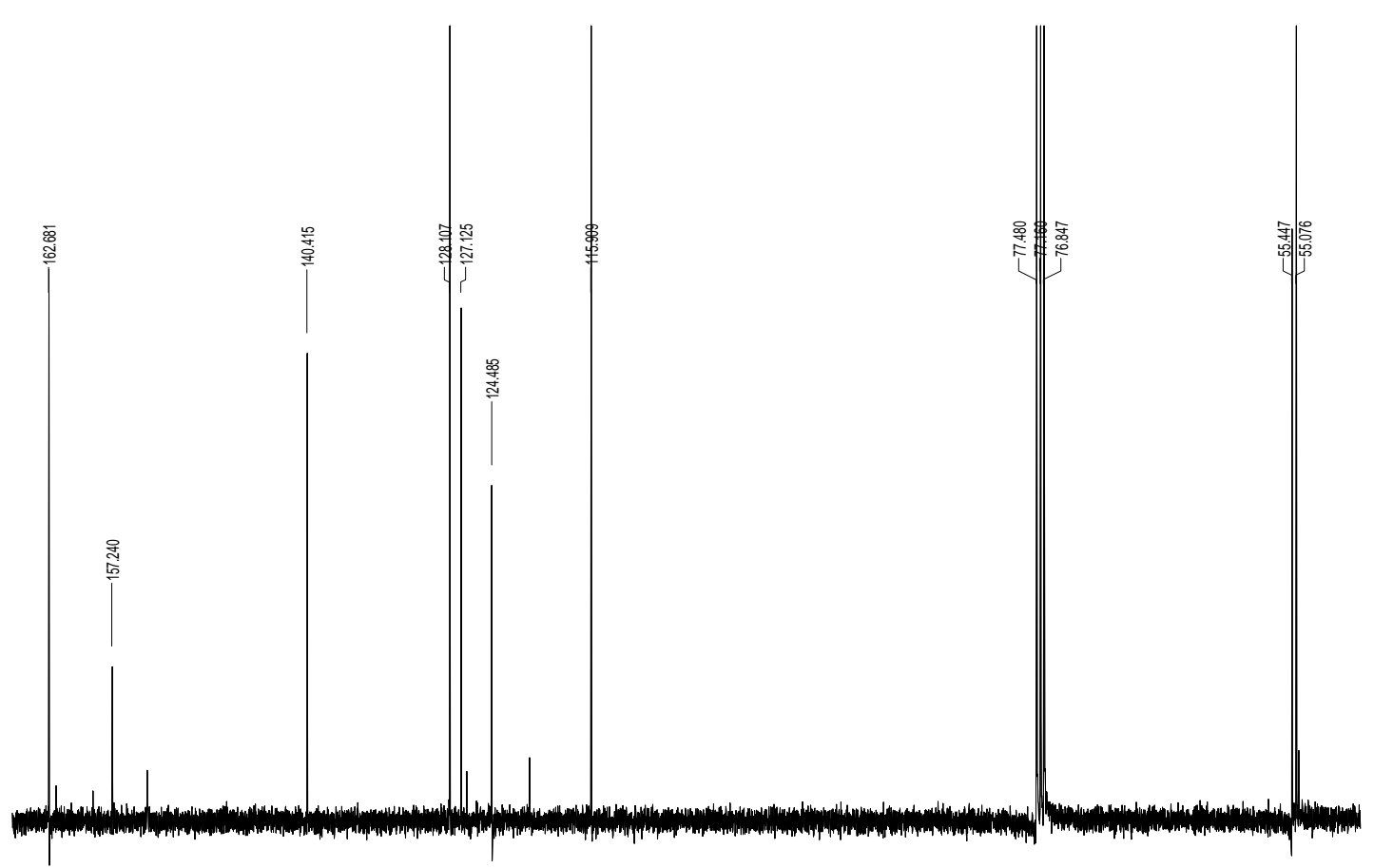




\section{3-(3,6-Dimethoxypyridazin-4-yl)-quinoline (16)}

${ }^{1} \mathrm{H}$ NMR (400 MHz, acetone- $\mathrm{d}_{6}$ )

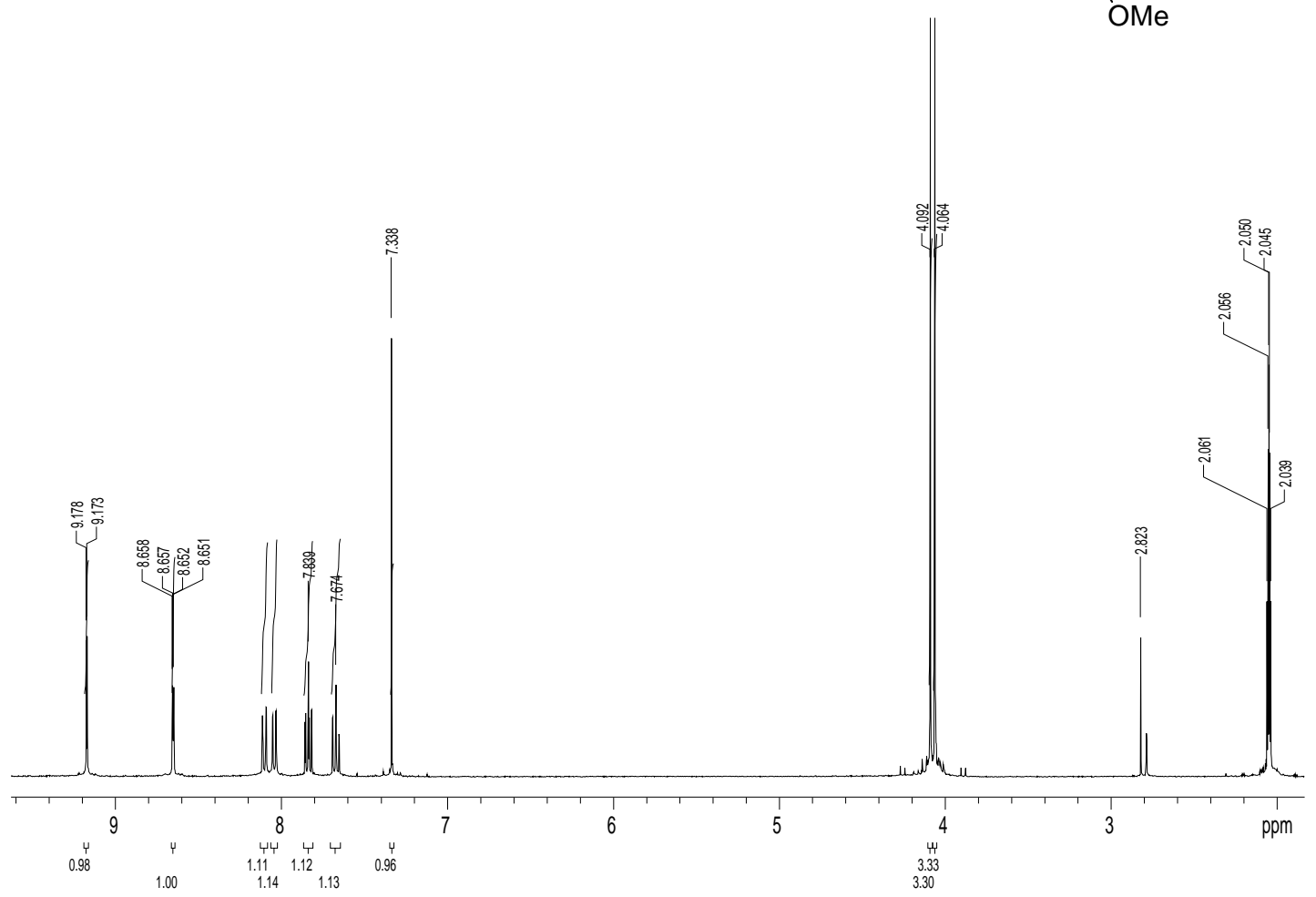

${ }^{13} \mathrm{C}$ NMR (100 MHz, acetone- $\mathrm{d}_{6}$ )

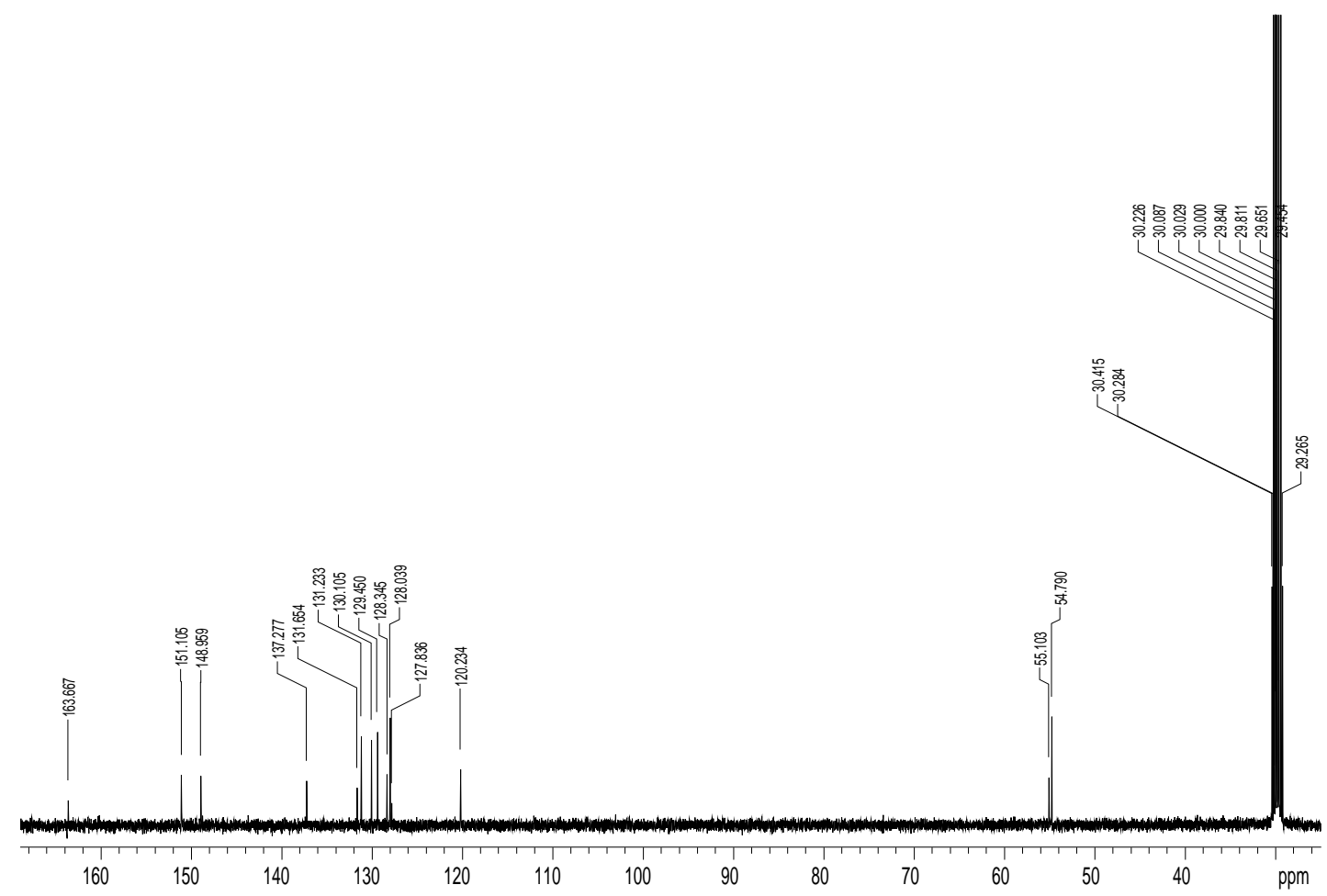


3-Methoxy-6-(2-methoxypyridin-5-yl)-pyridazine (18c)

${ }^{1} \mathrm{H}$ NMR (400 MHz, $\mathrm{CDCl}_{3}$ )

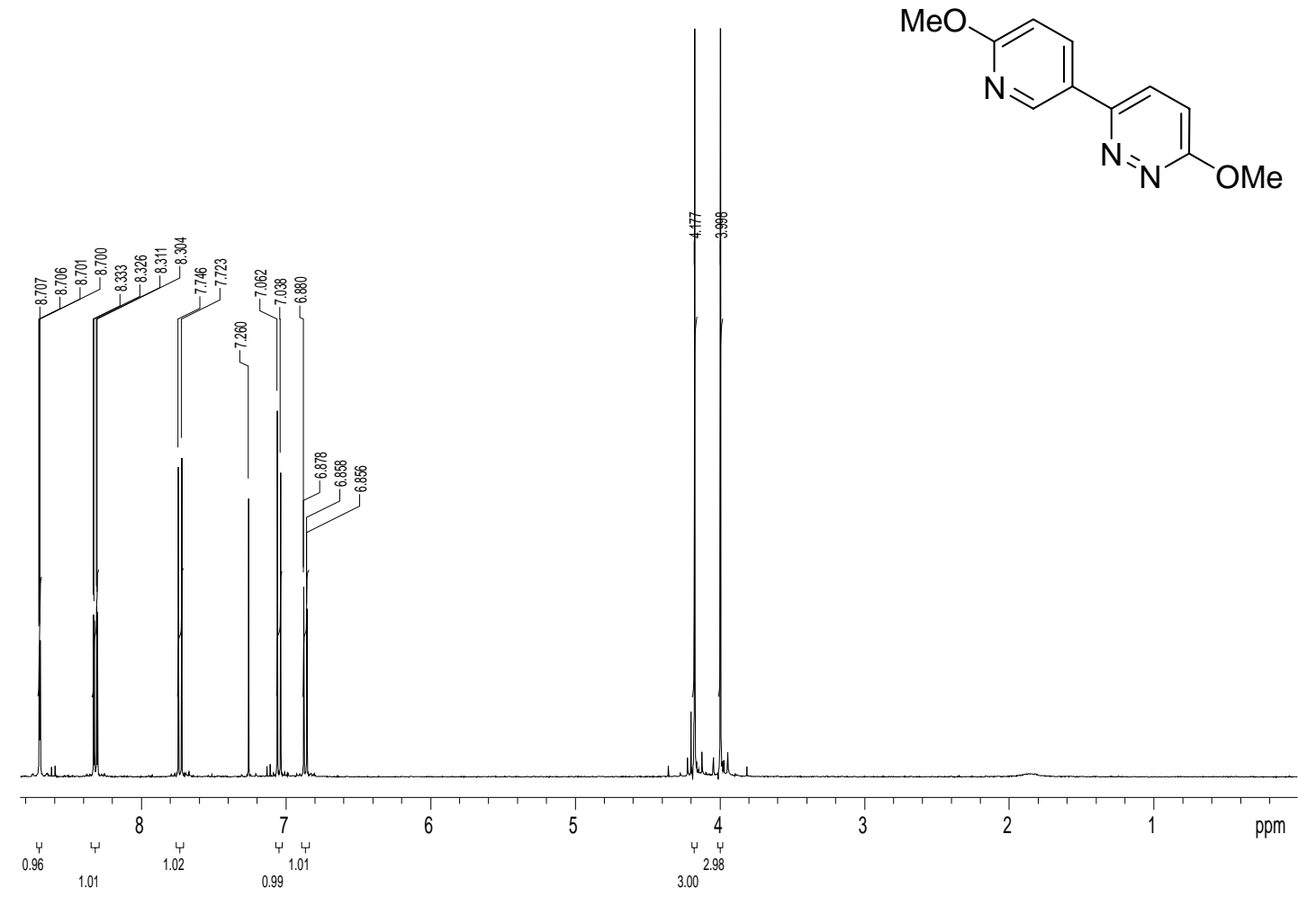

${ }^{13} \mathrm{C}$ NMR $\left(100 \mathrm{MHz}, \mathrm{CDCl}_{3}\right)$

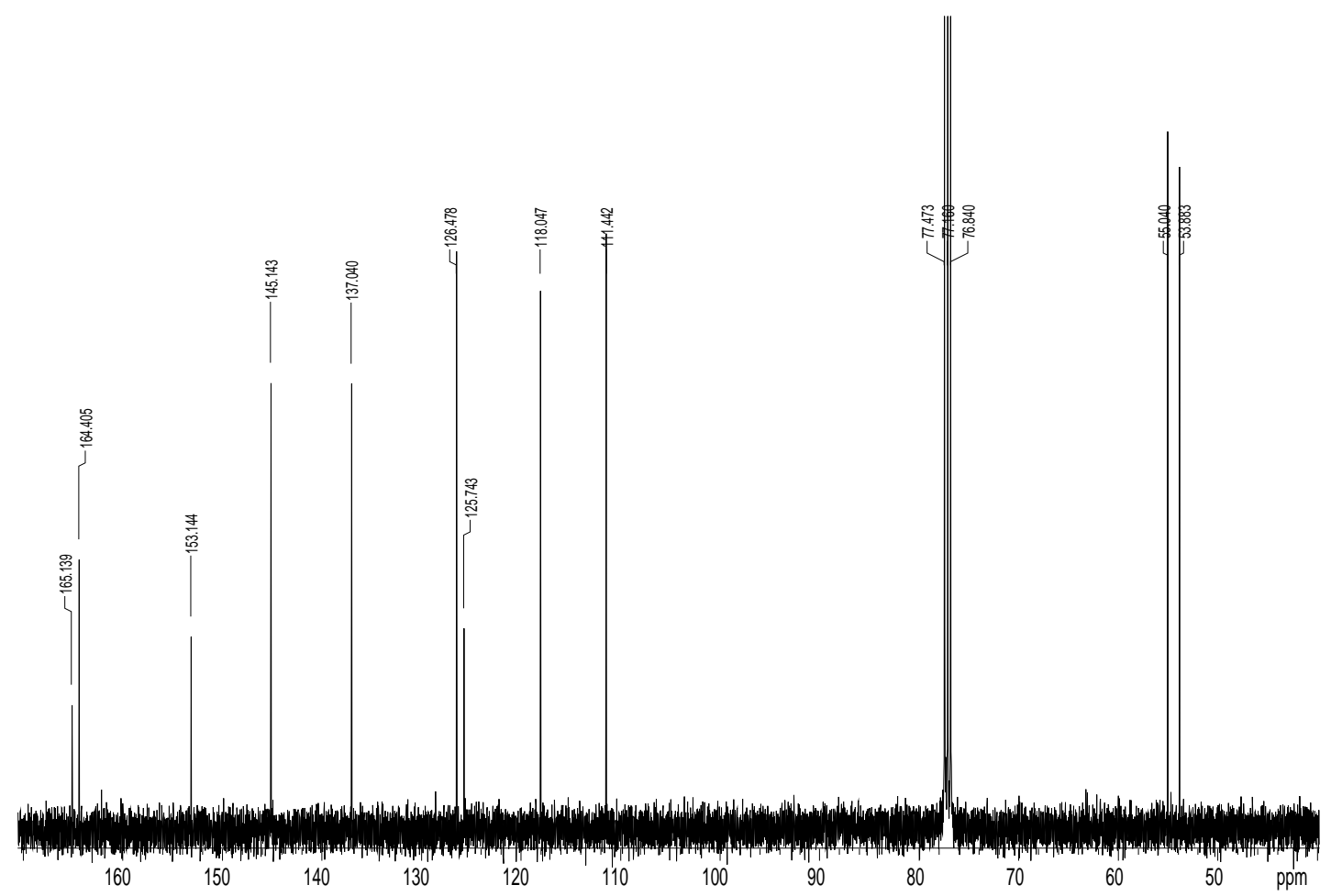


3-Methoxy-6-(2-fluoropyridin-5-yl)-pyridazine (18d)

${ }^{1} \mathrm{H}$ NMR (400 MHz, $\mathrm{CDCl}_{3}$ )

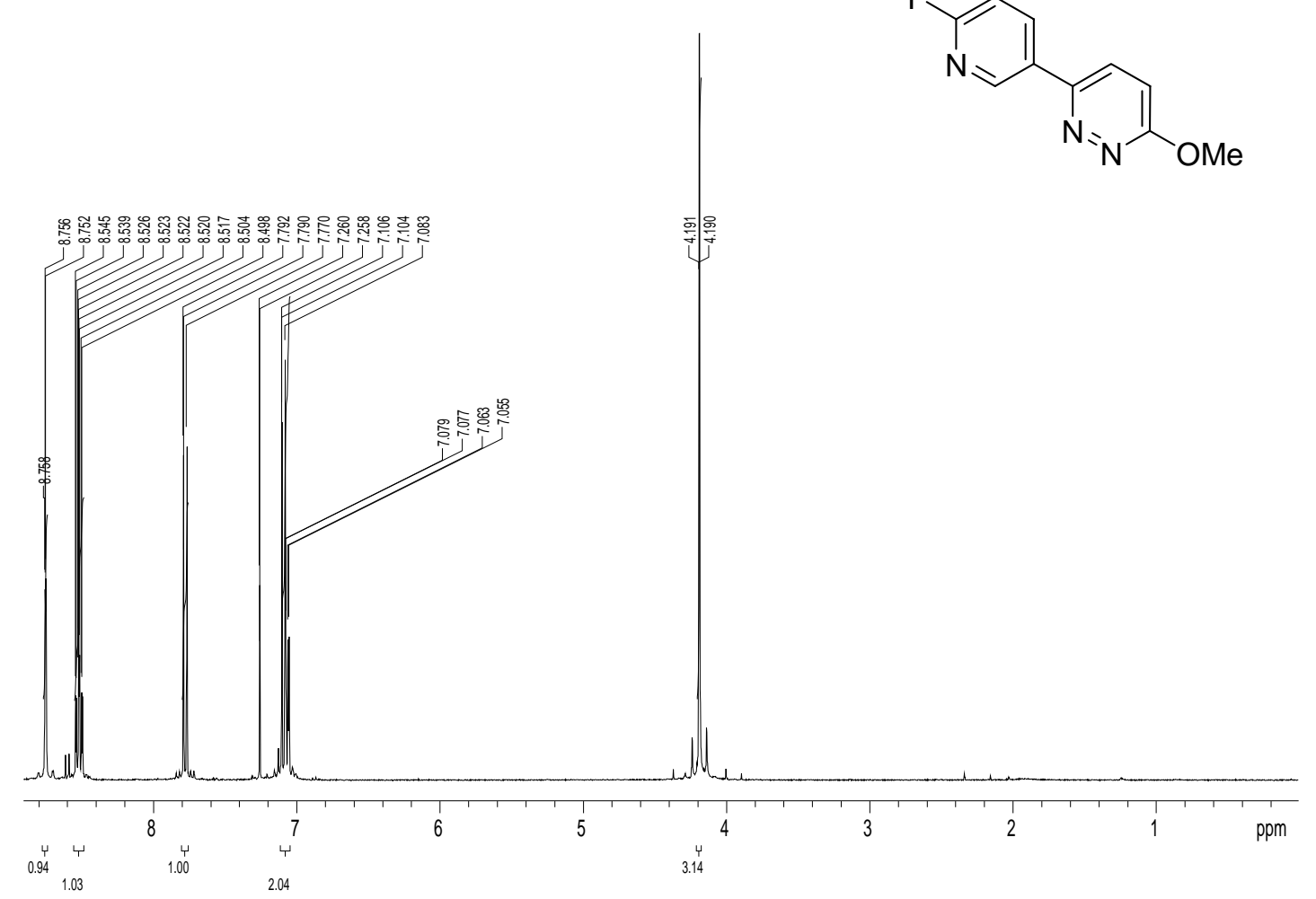

${ }^{13} \mathrm{C}$ NMR (100 MHz, $\mathrm{CDCl}_{3}$ )

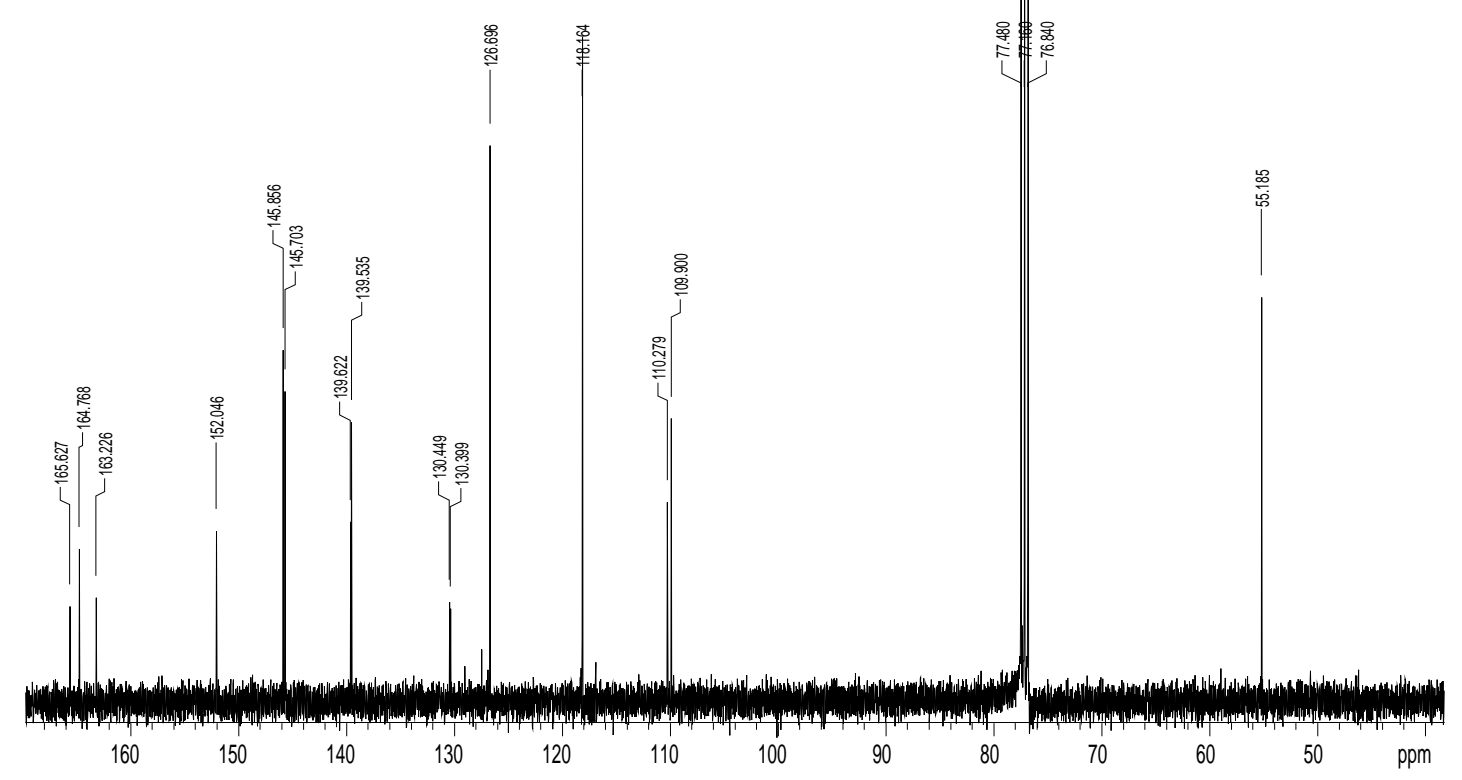


3-Methoxy-6-phenyl-4-pyridazinylboronic acid (19a)

${ }^{1} \mathrm{H}$ NMR (400 MHz, DMSO-d ${ }_{6}$ )

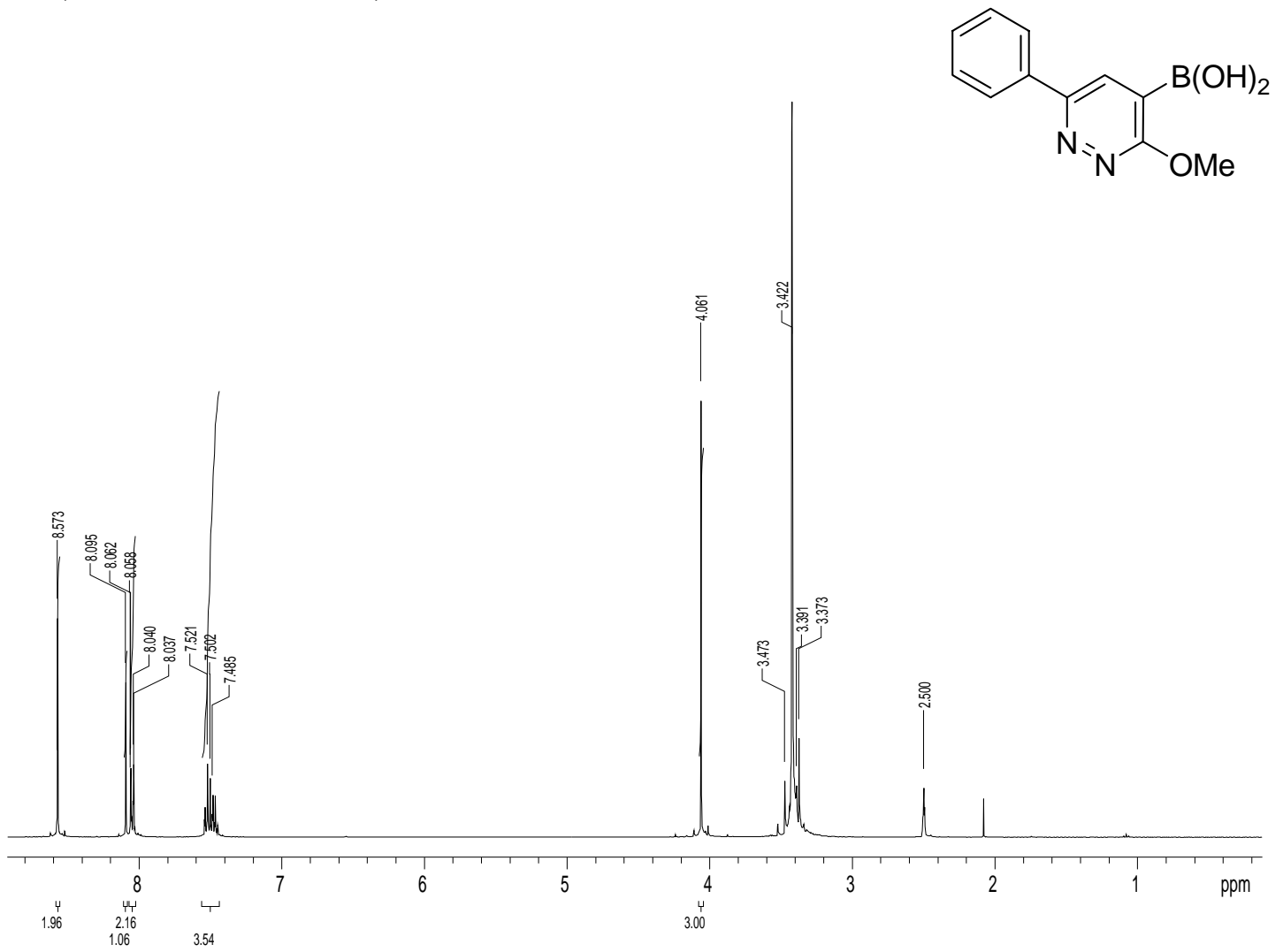

${ }^{13} \mathrm{C}$ NMR (100 MHz, DMSO-d 6 )

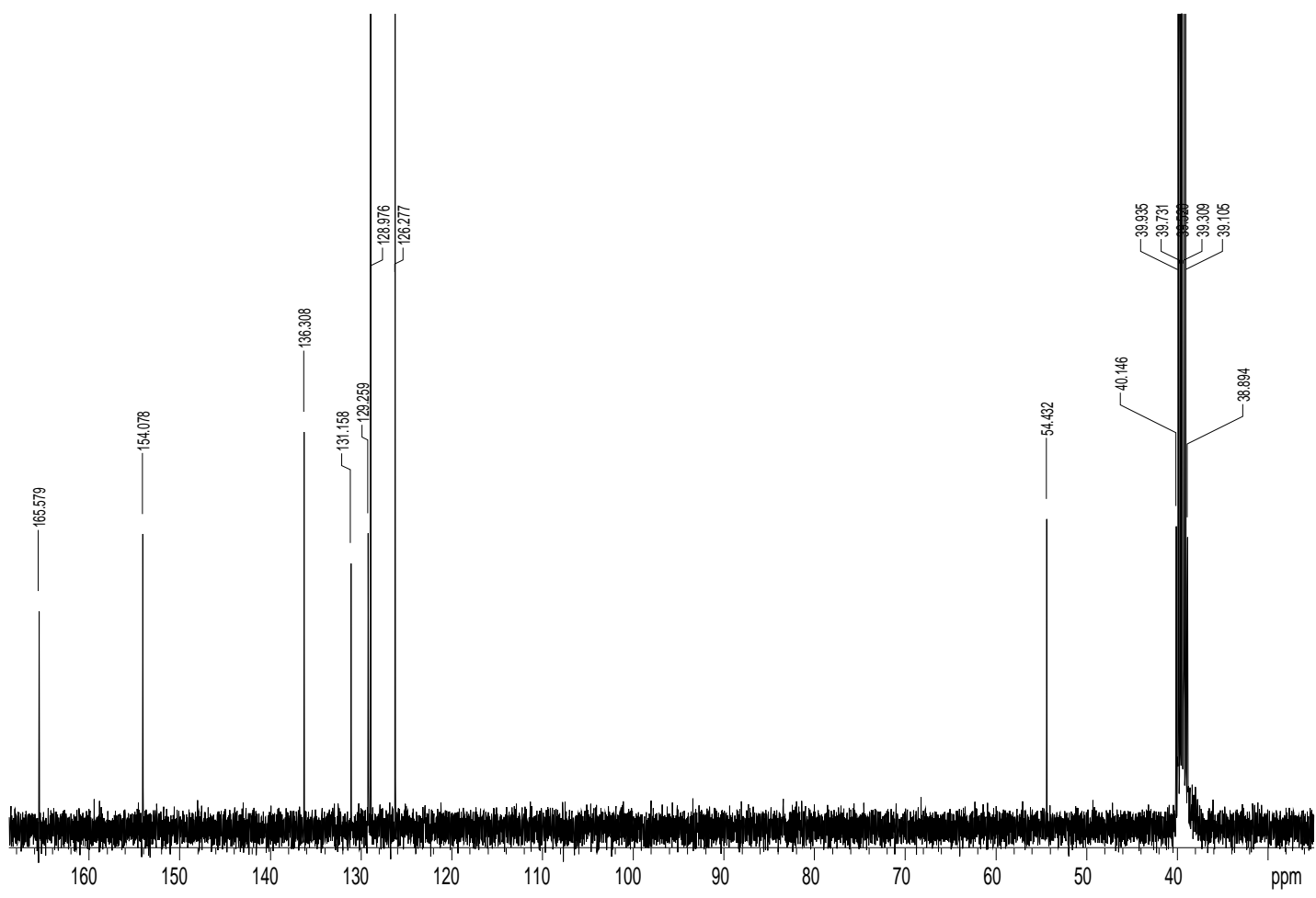


3-Methoxy-6-(4-methoxyphenyl)-4-pyridazinylboronic acid (19b)

${ }^{1} \mathrm{H}$ NMR (400 MHz, DMSO-d ${ }_{6}$ )

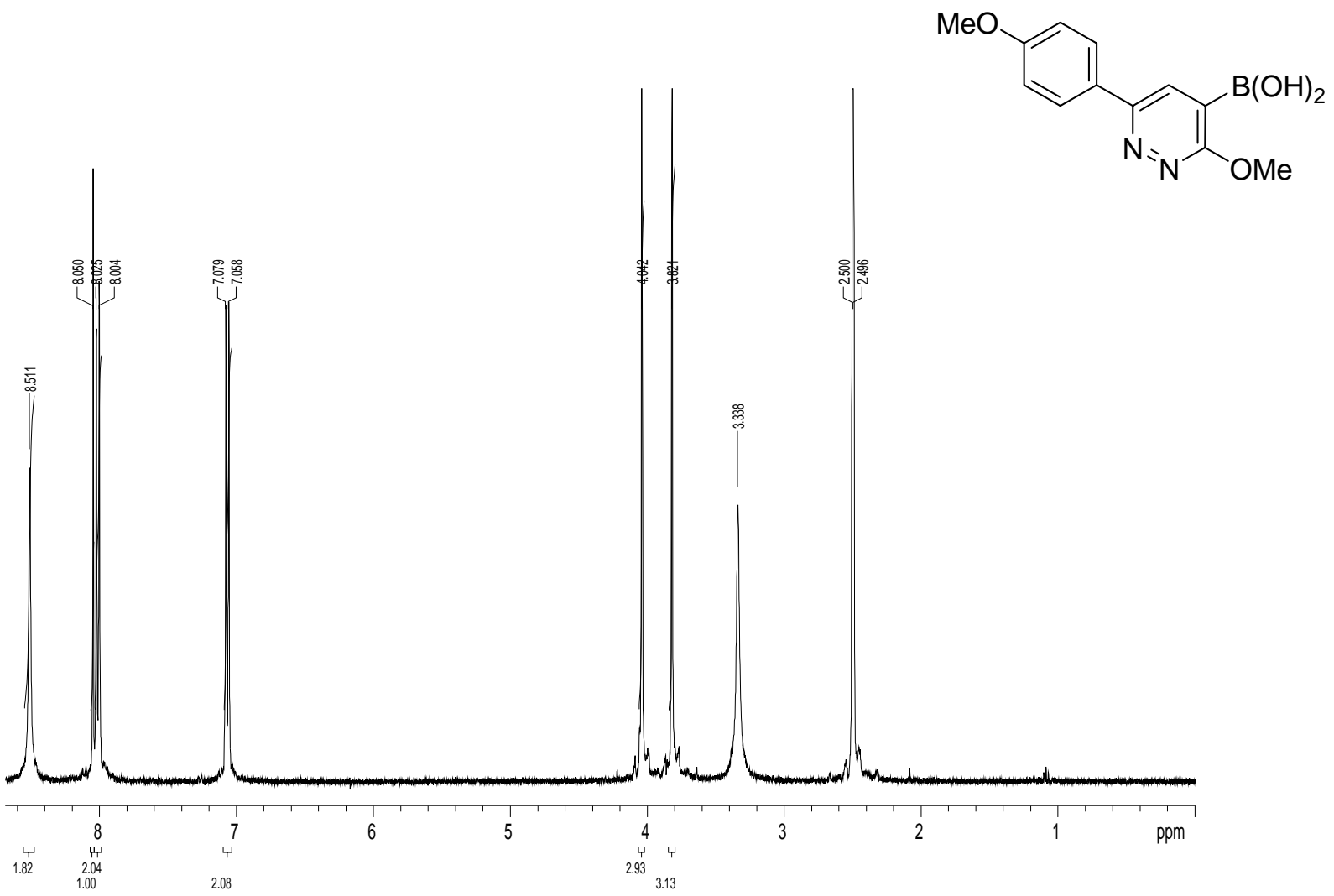

${ }^{13} \mathrm{C}$ NMR (100 MHz, DMSO-d 6 )

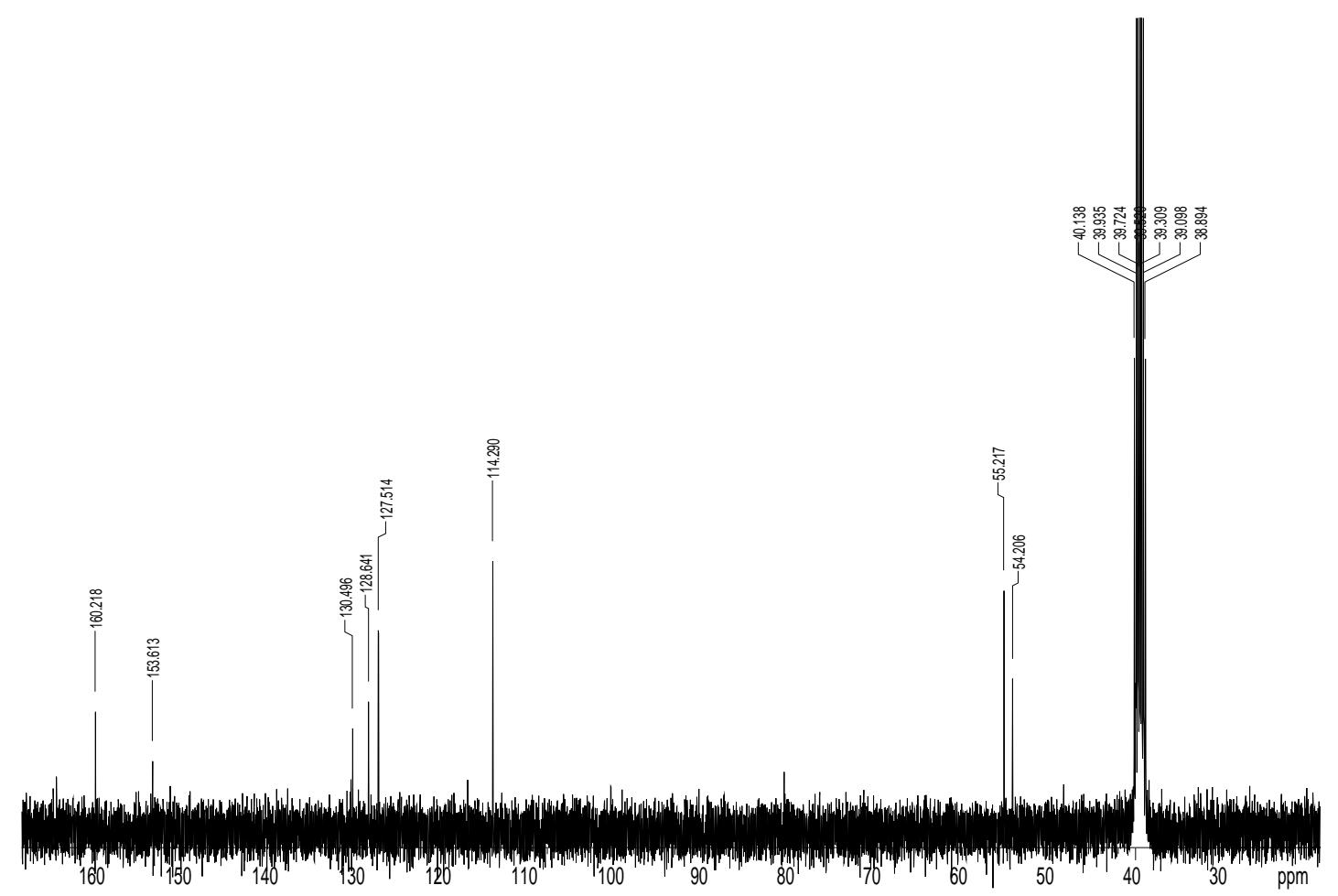


3-Methoxy-6-(2-methoxypyridin-5-yl)-4-pyridazinylboronic acid (19c)

${ }^{1} \mathrm{H}$ NMR (400 MHz, DMSO-d $\left._{6}\right)$

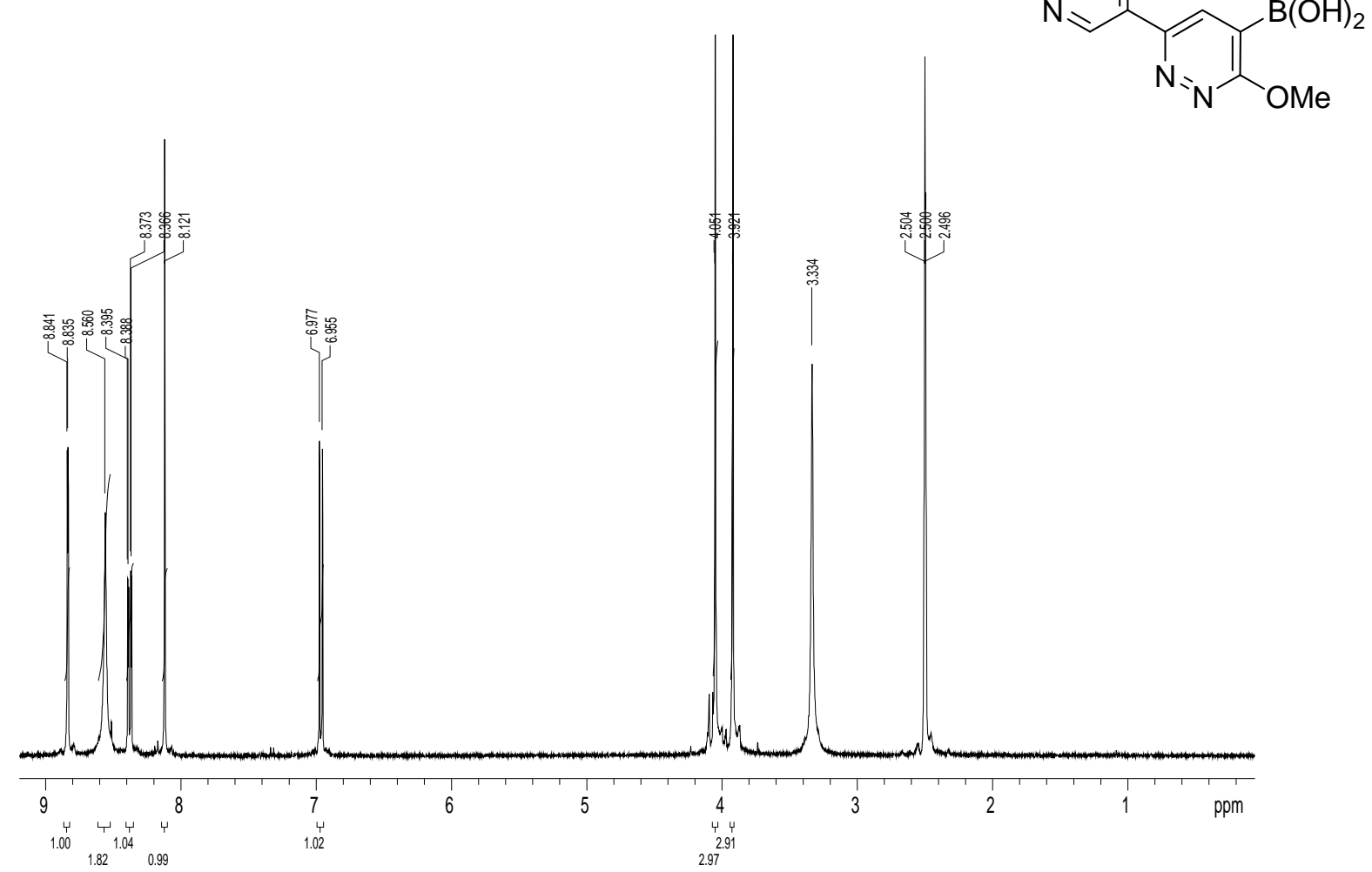

${ }^{13} \mathrm{C}$ NMR (100 MHz, DMSO-d 6 )

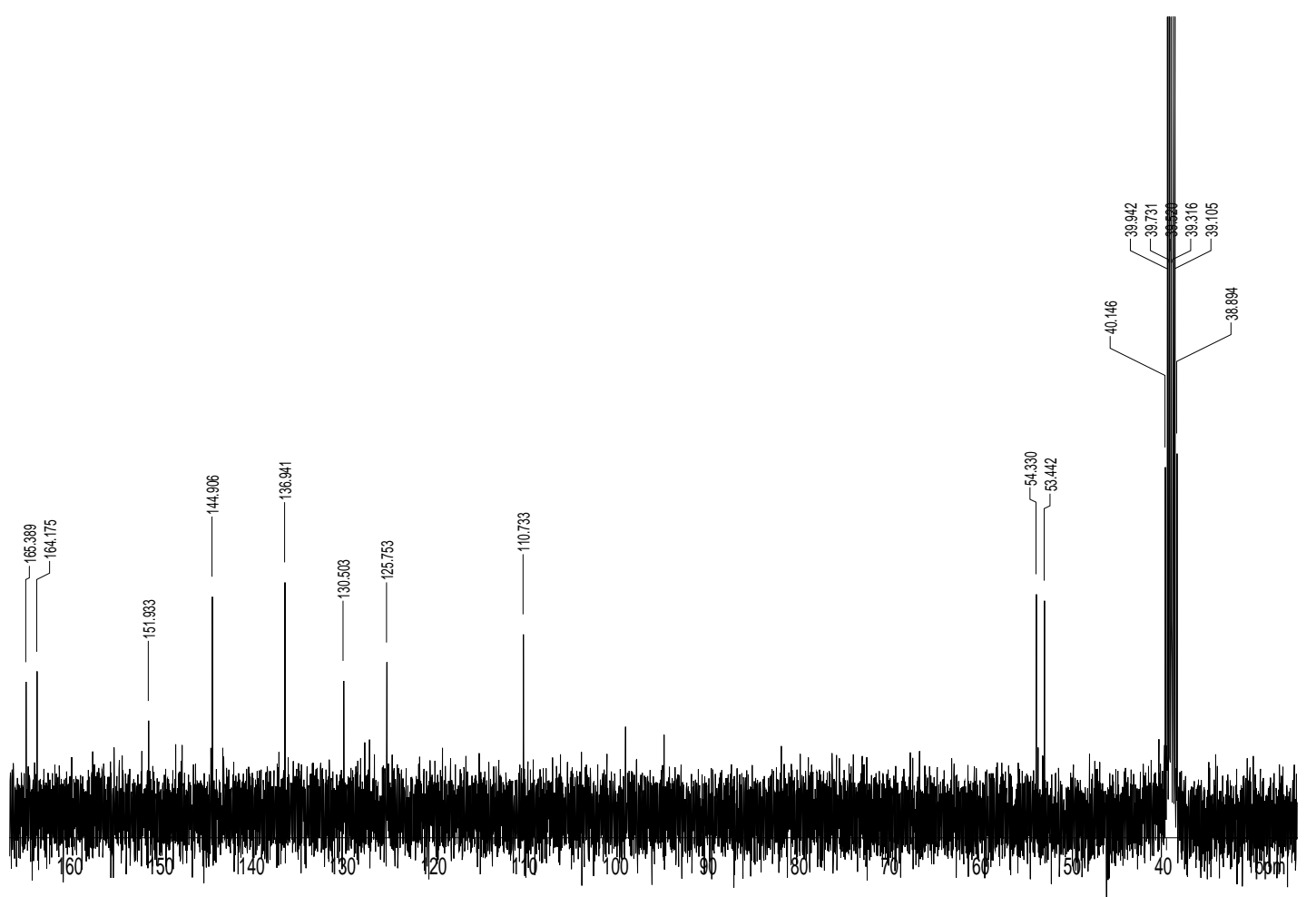


3-Methoxy-6-(2-fluoropyridin-5-yl)-4-pyridazinylboronic acid (19d)

${ }^{1} \mathrm{H}$ NMR (400 MHz, DMSO-d )

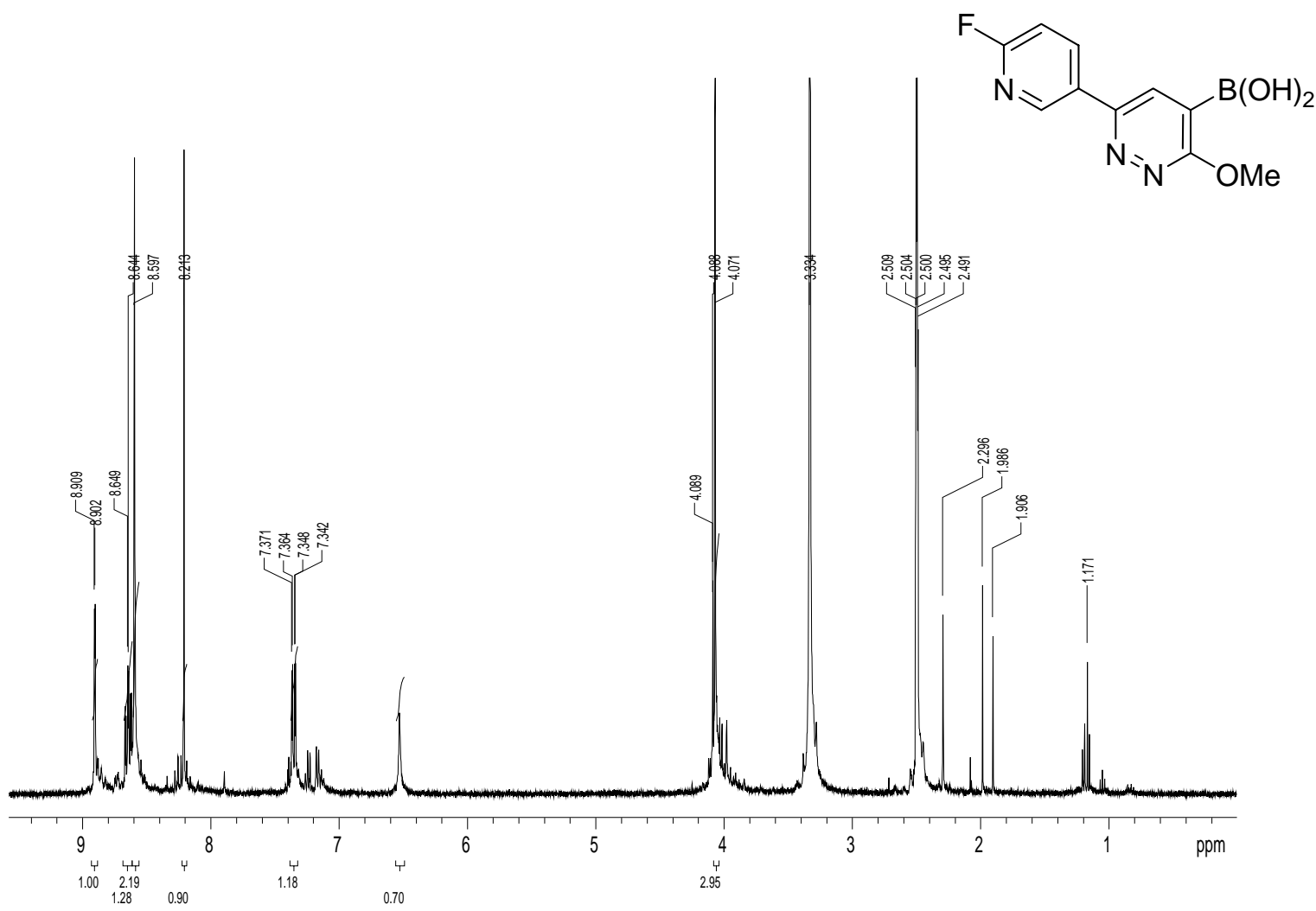

${ }^{13} \mathrm{C}$ NMR (100 MHz, DMSO-d 6 )

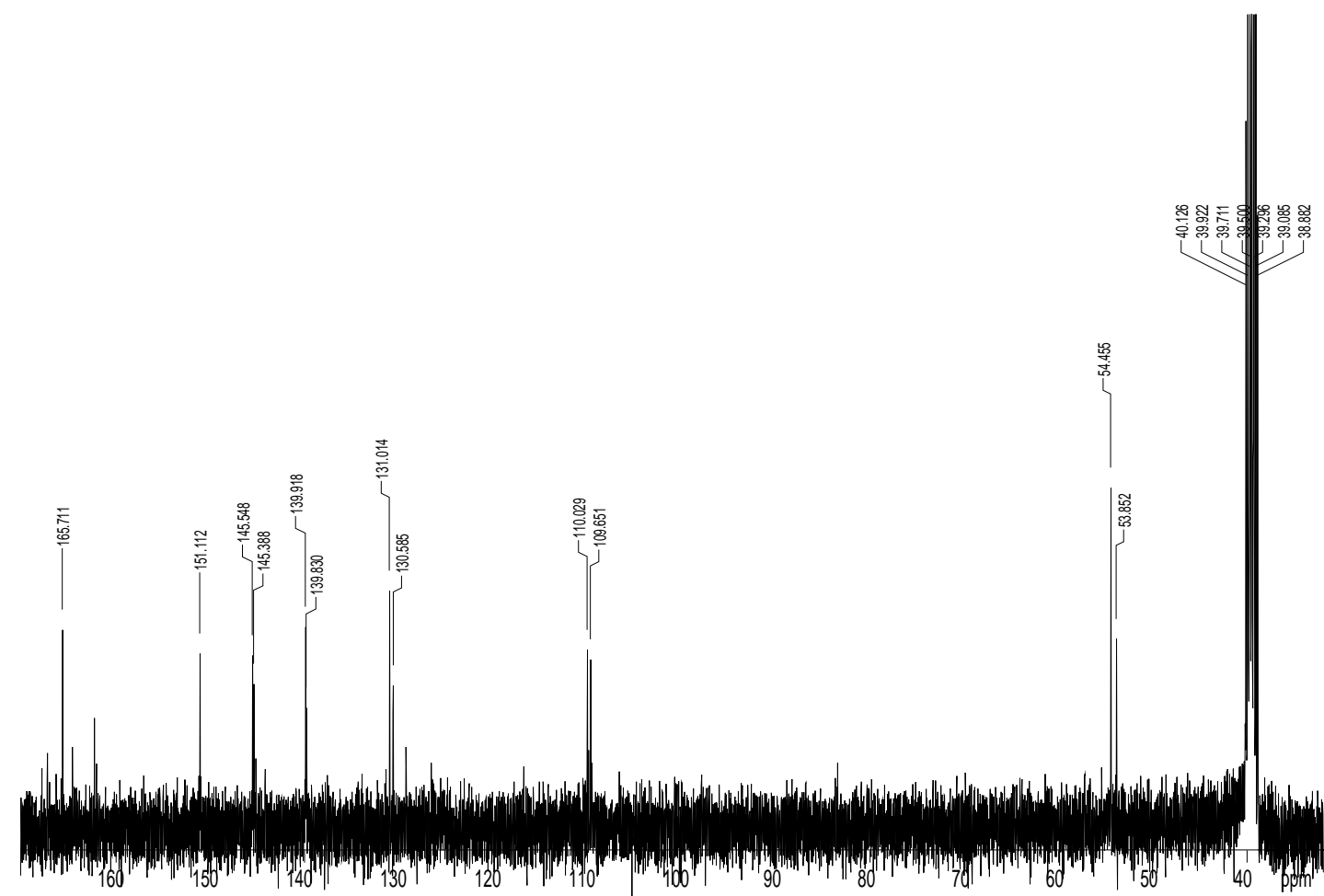


3-Methoxy-6-phenyl-4-(4,4,5,5-tetramethyl-1,3,2-dioxaborolan-2-yl)pyridazine (20a) ${ }^{1} \mathrm{H}$ NMR (400 MHz, $\mathrm{CDCl}_{3}$ )

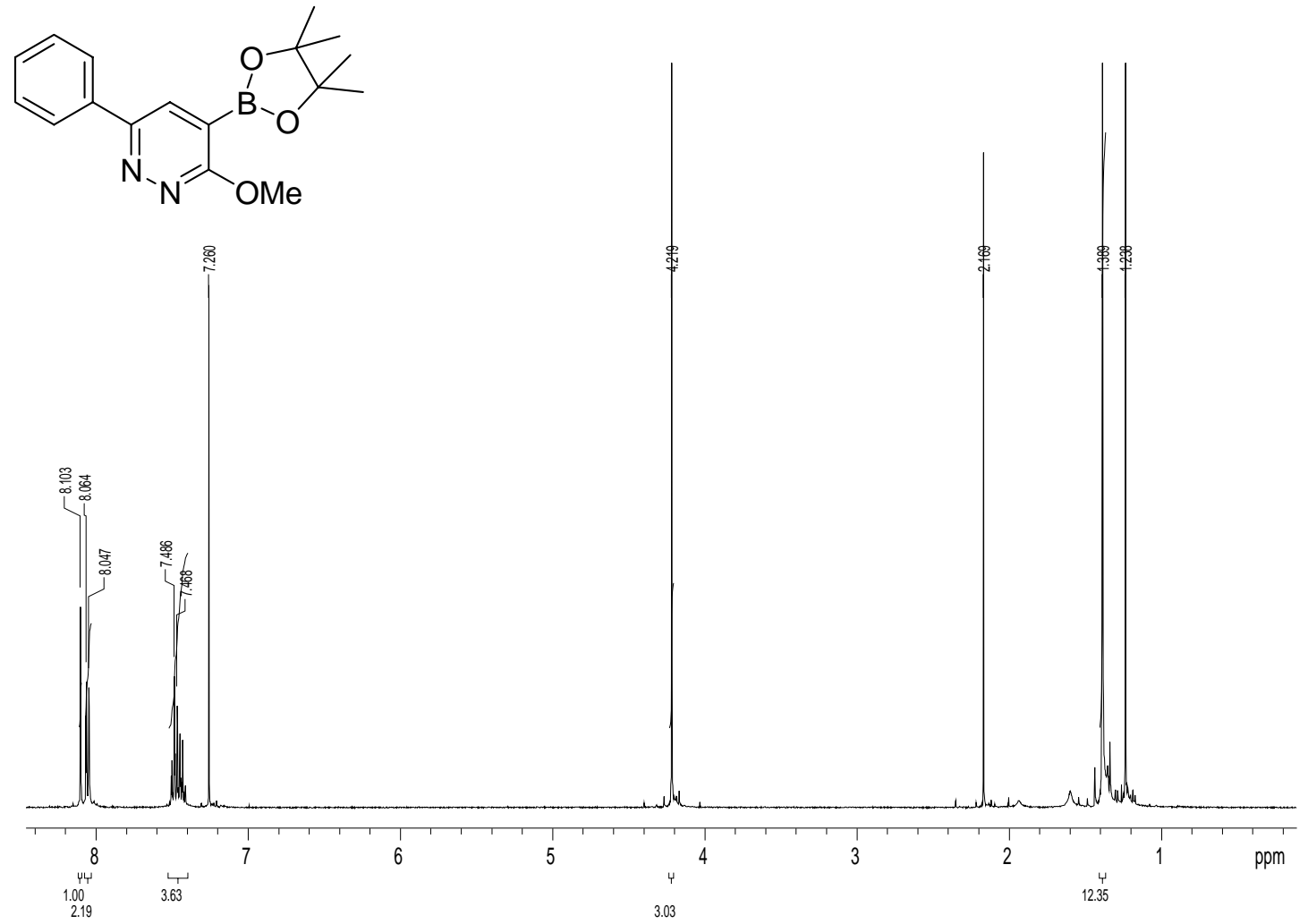

${ }^{13} \mathrm{C}$ NMR $\left(100 \mathrm{MHz}, \mathrm{CDCl}_{3}\right)$

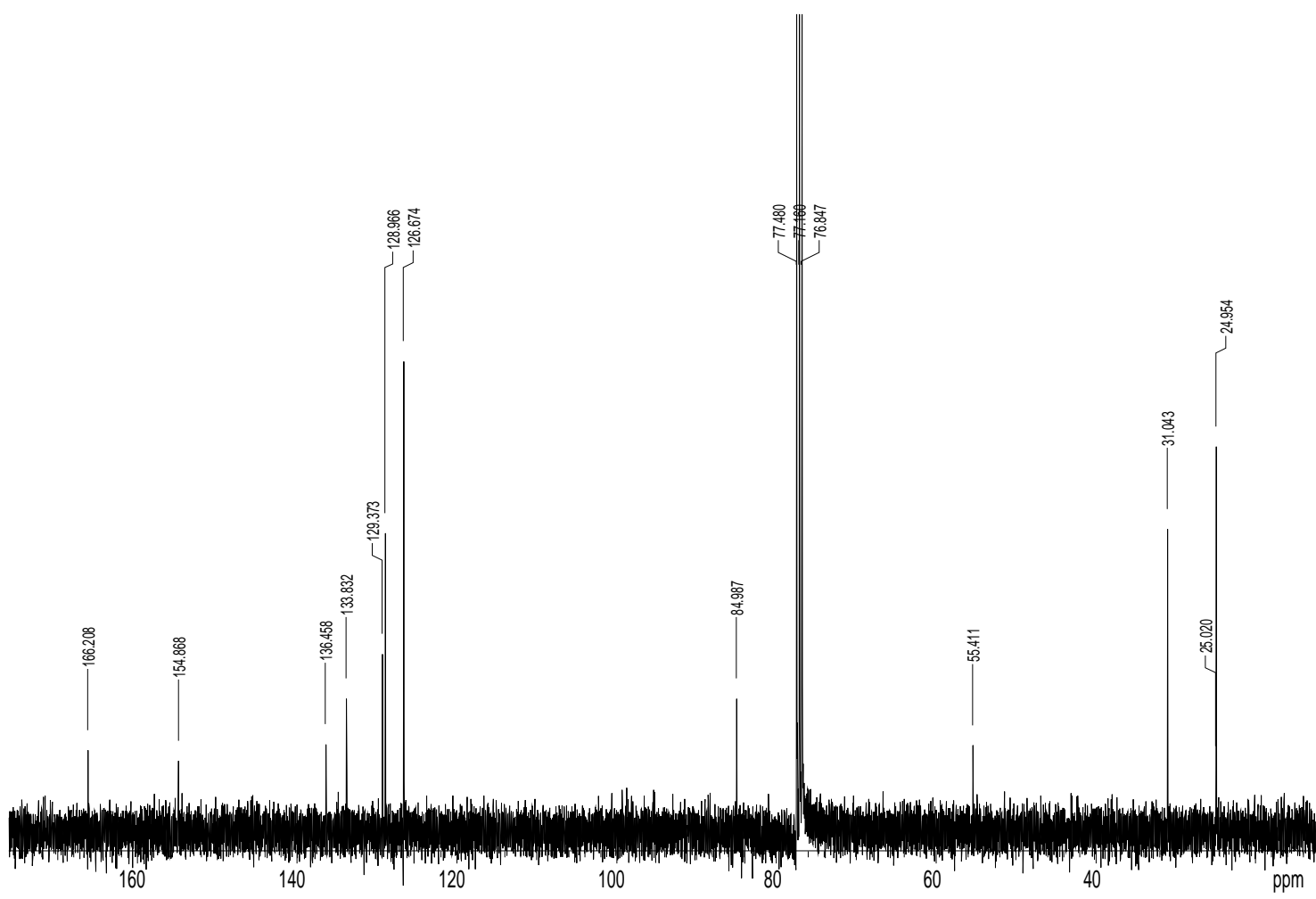


3-Methoxy-6-(4-methoxyphenyl)-4-(4,4,5,5-tetramethyl-1,3,2-dioxaborolan-2-yl)pyridazine (20b)

${ }^{1} \mathrm{H}$ NMR $\left(400 \mathrm{MHz}, \mathrm{CDCl}_{3}\right)$

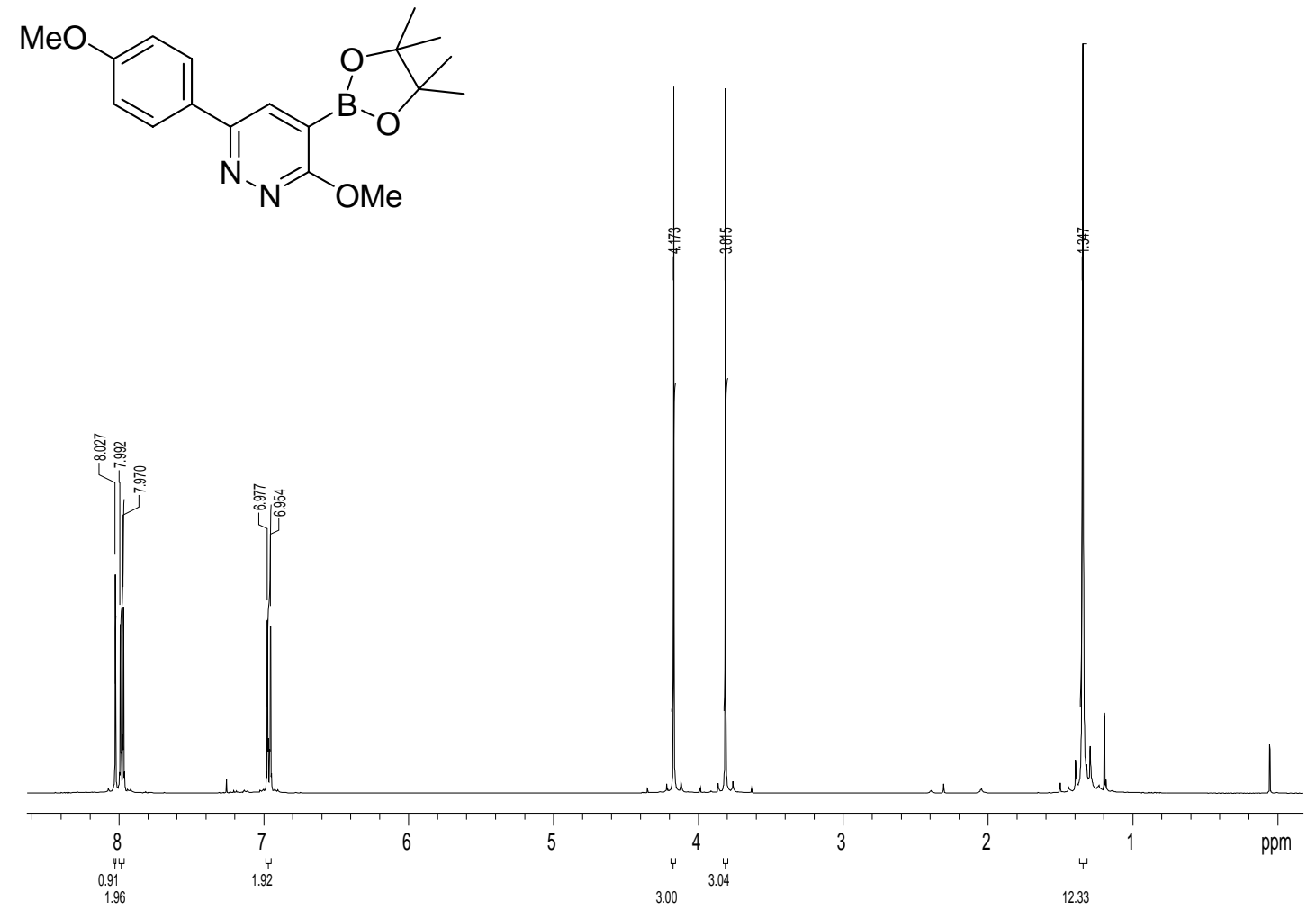

${ }^{13} \mathrm{C}$ NMR $\left(100 \mathrm{MHz}, \mathrm{CDCl}_{3}\right)$

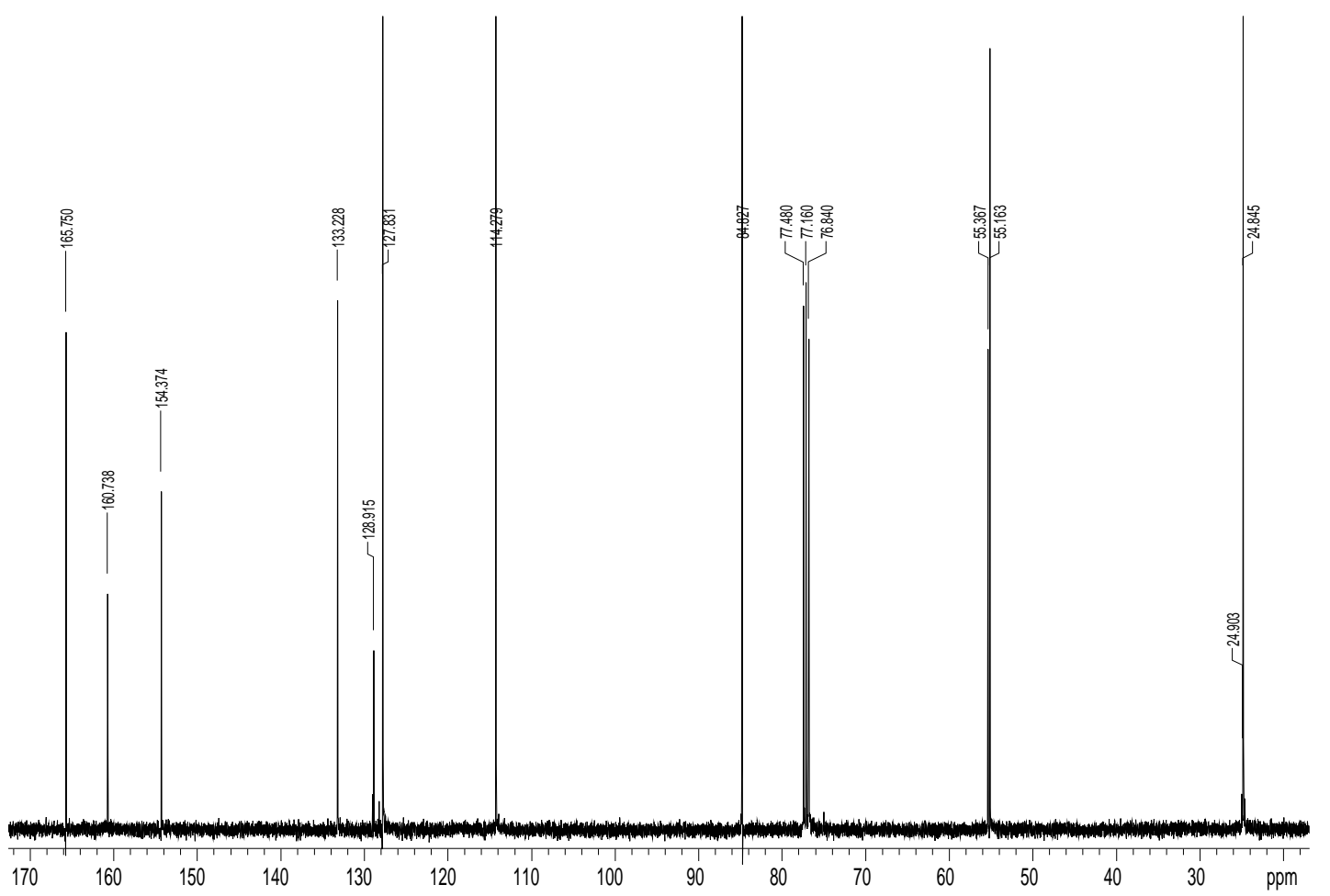


3-Methoxy-6-(2-methoxypyridin-5-yl)-4-(4,4,5,5-tetramethyl-1,3,2-dioxaborolan-2-

yl)pyridazine (20c)

${ }^{1} \mathrm{H} \mathrm{NMR}\left(400 \mathrm{MHz}, \mathrm{CDCl}_{3}\right)$

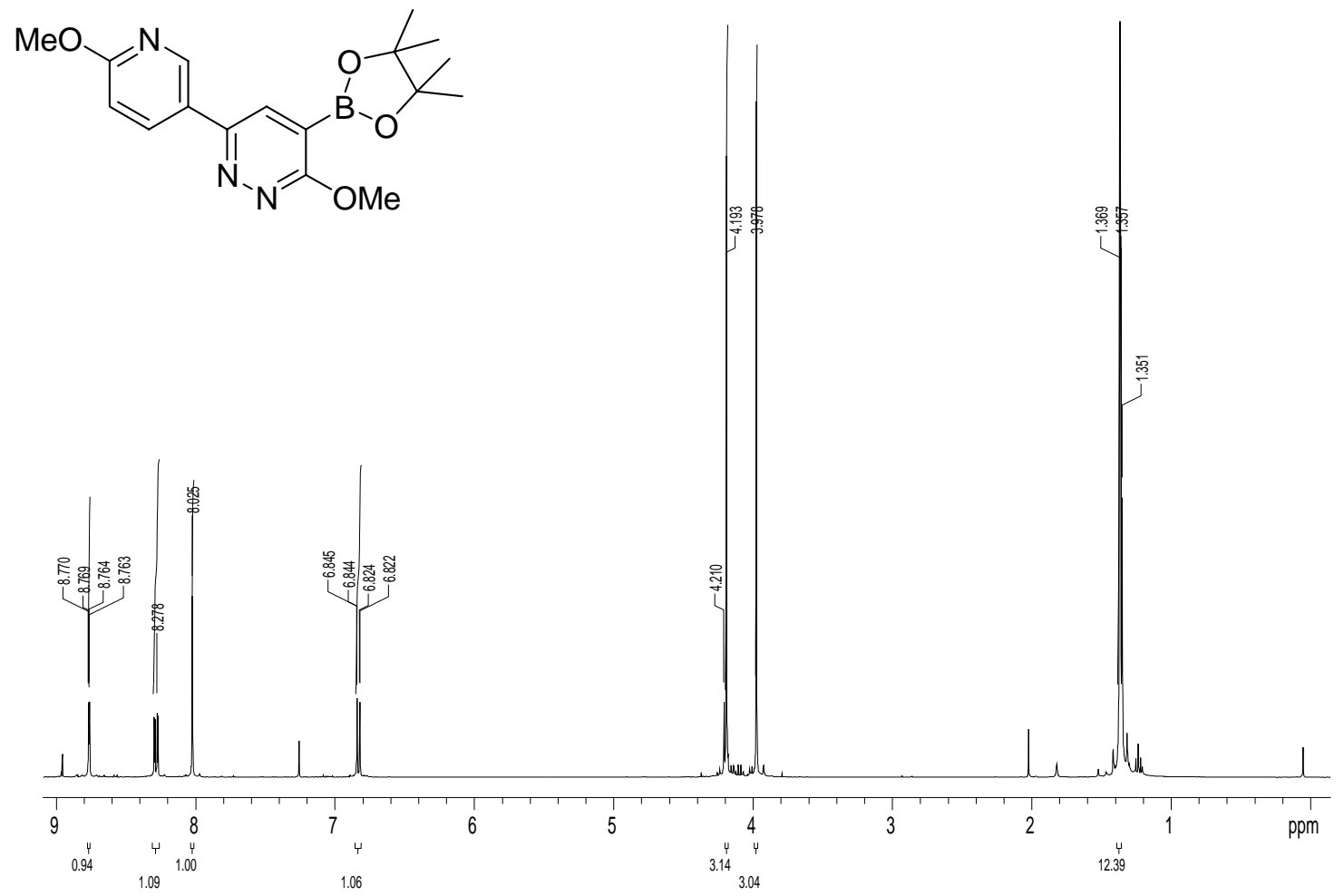

${ }^{13} \mathrm{C}$ NMR $\left(100 \mathrm{MHz}, \mathrm{CDCl}_{3}\right)$

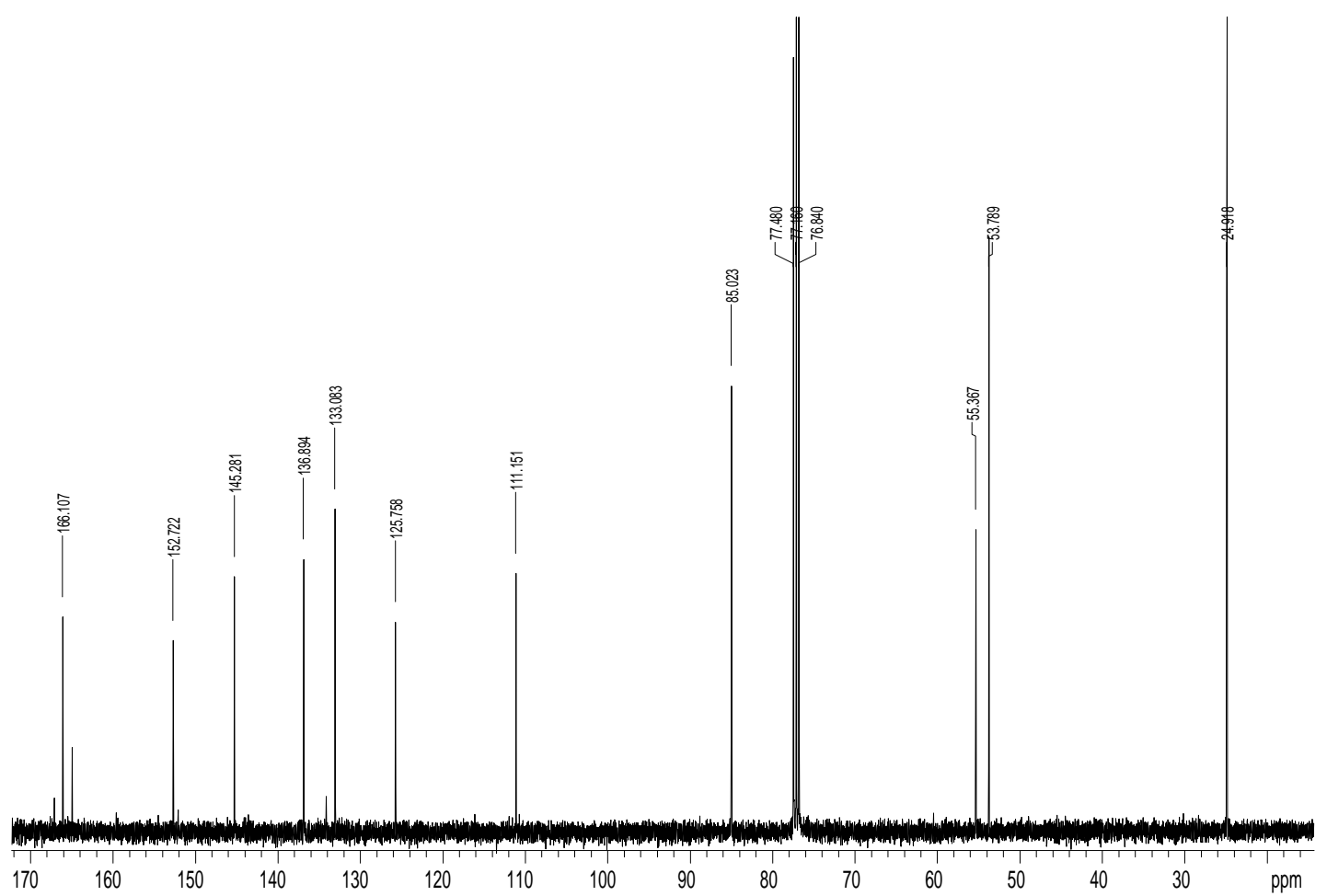


3-Methoxy-6-(2-fluoropyridin-5-yl)-4-(4,4,5,5-tetramethyl-1,3,2-dioxaborolan-2-yl)pyridazine (20d)

${ }^{1} \mathrm{H}$ NMR $\left(400 \mathrm{MHz}, \mathrm{CDCl}_{3}\right)$<smiles>COc1nnc(-c2ccc(F)nc2)cc1B1OC2(C)CC1(C)C2</smiles>

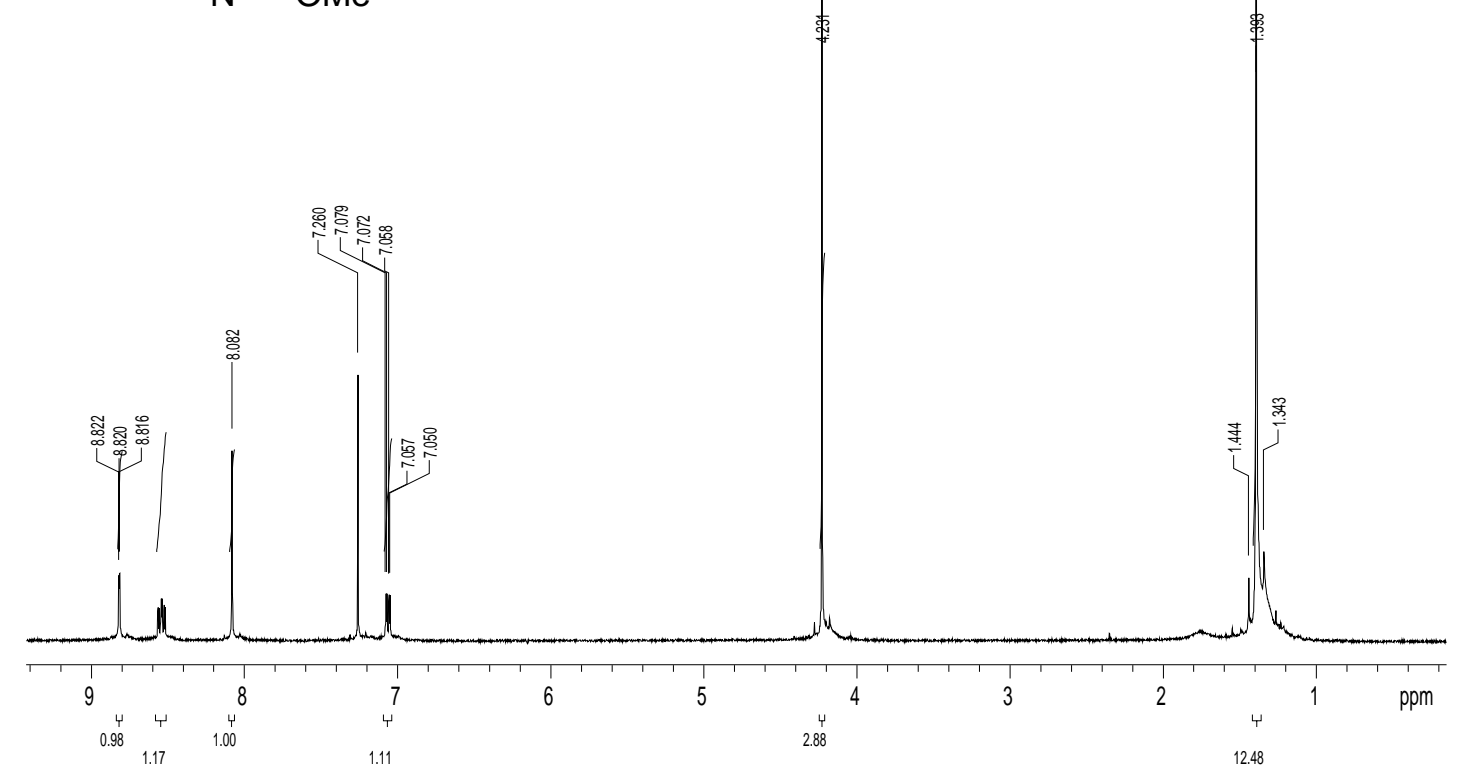

${ }^{13} \mathrm{C}$ NMR (100 MHz, $\mathrm{CDCl}_{3}$ )

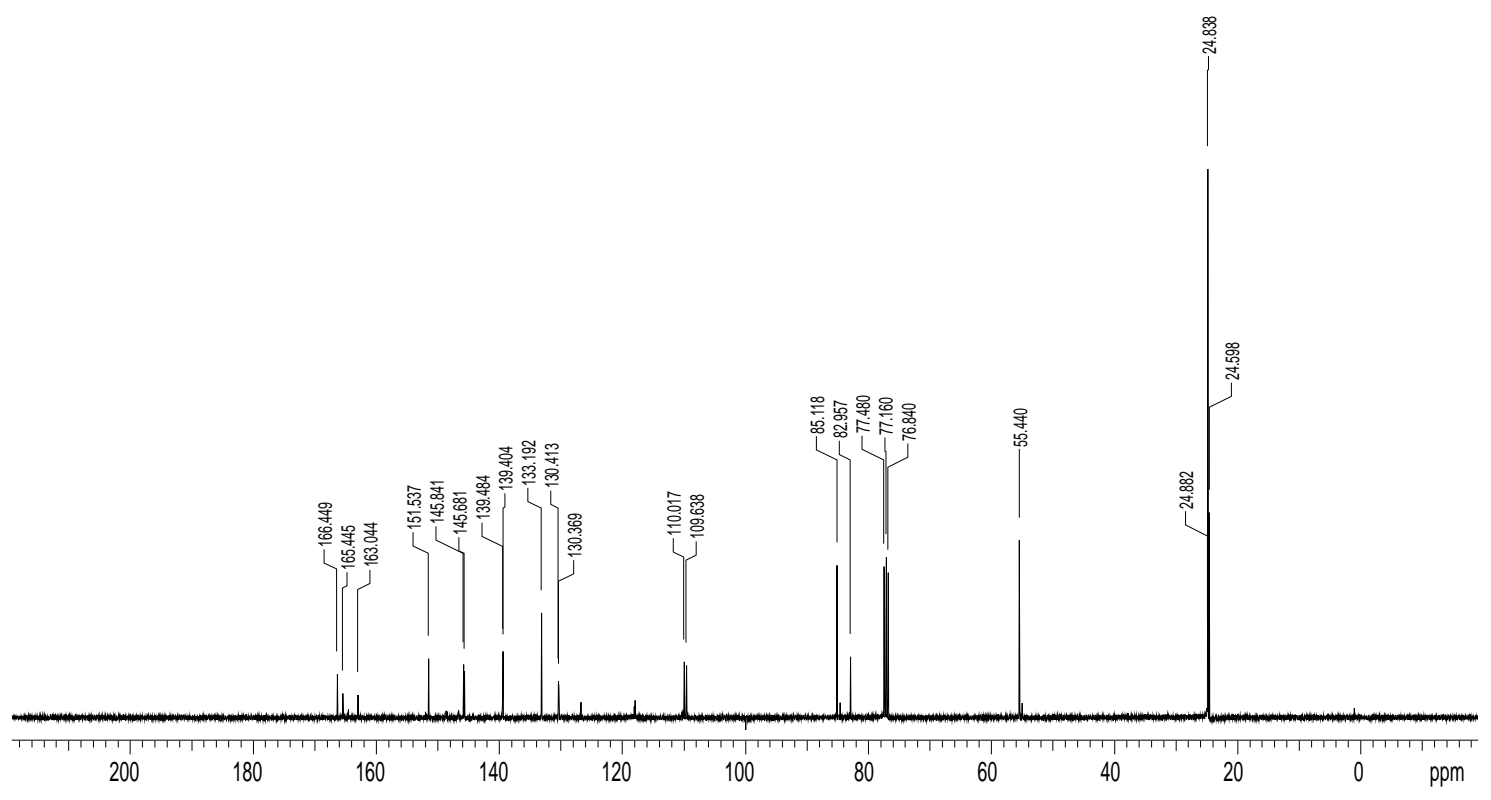


3-Methoxy-6-phenyl-4-(pyridin-2-yl)pyridazine (21)

${ }^{1} \mathrm{H}$ NMR (400 MHz, $\mathrm{CDCl}_{3}$ )

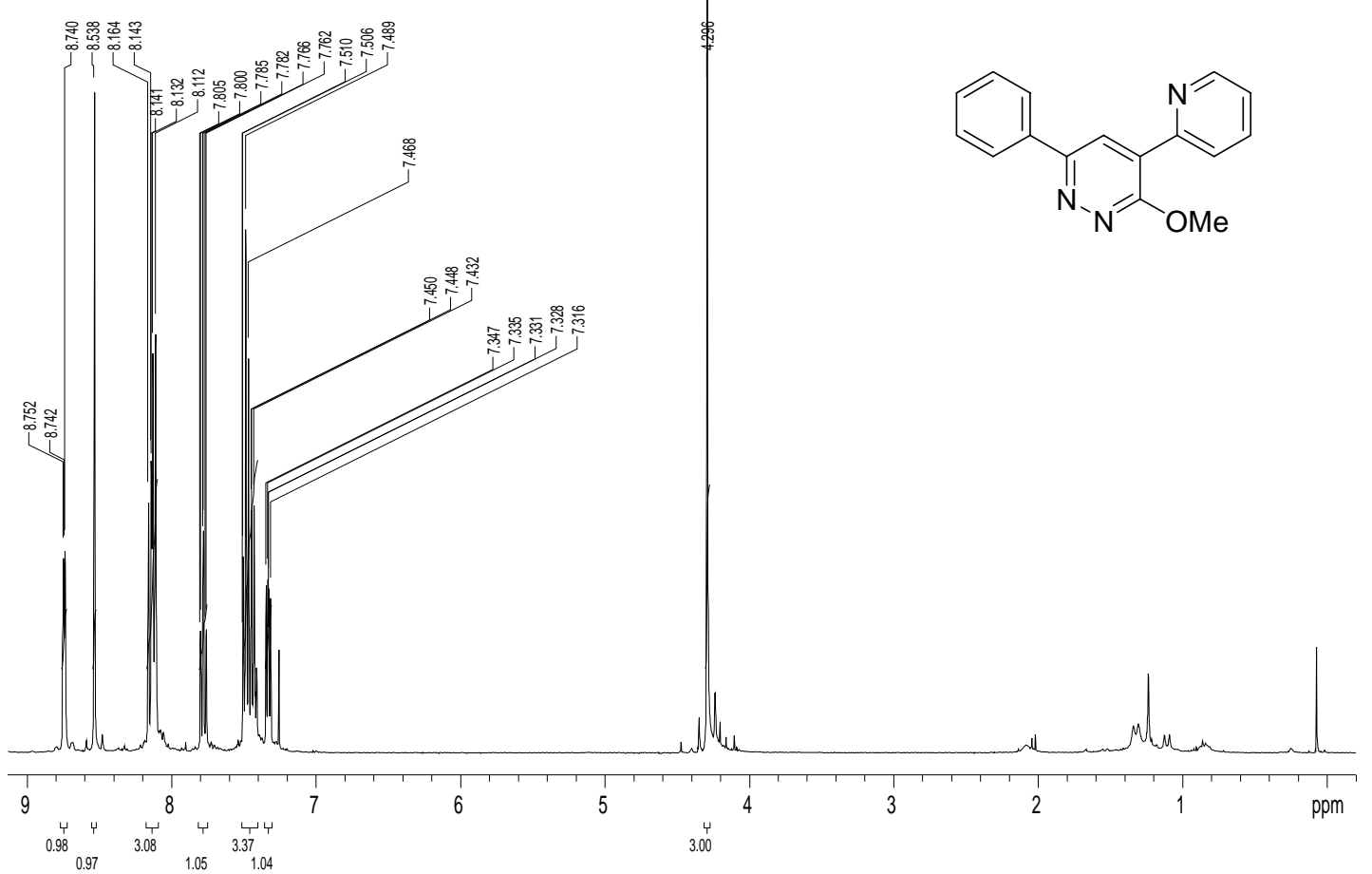

${ }^{13} \mathrm{C}$ NMR $\left(100 \mathrm{MHz}, \mathrm{CDCl}_{3}\right)$

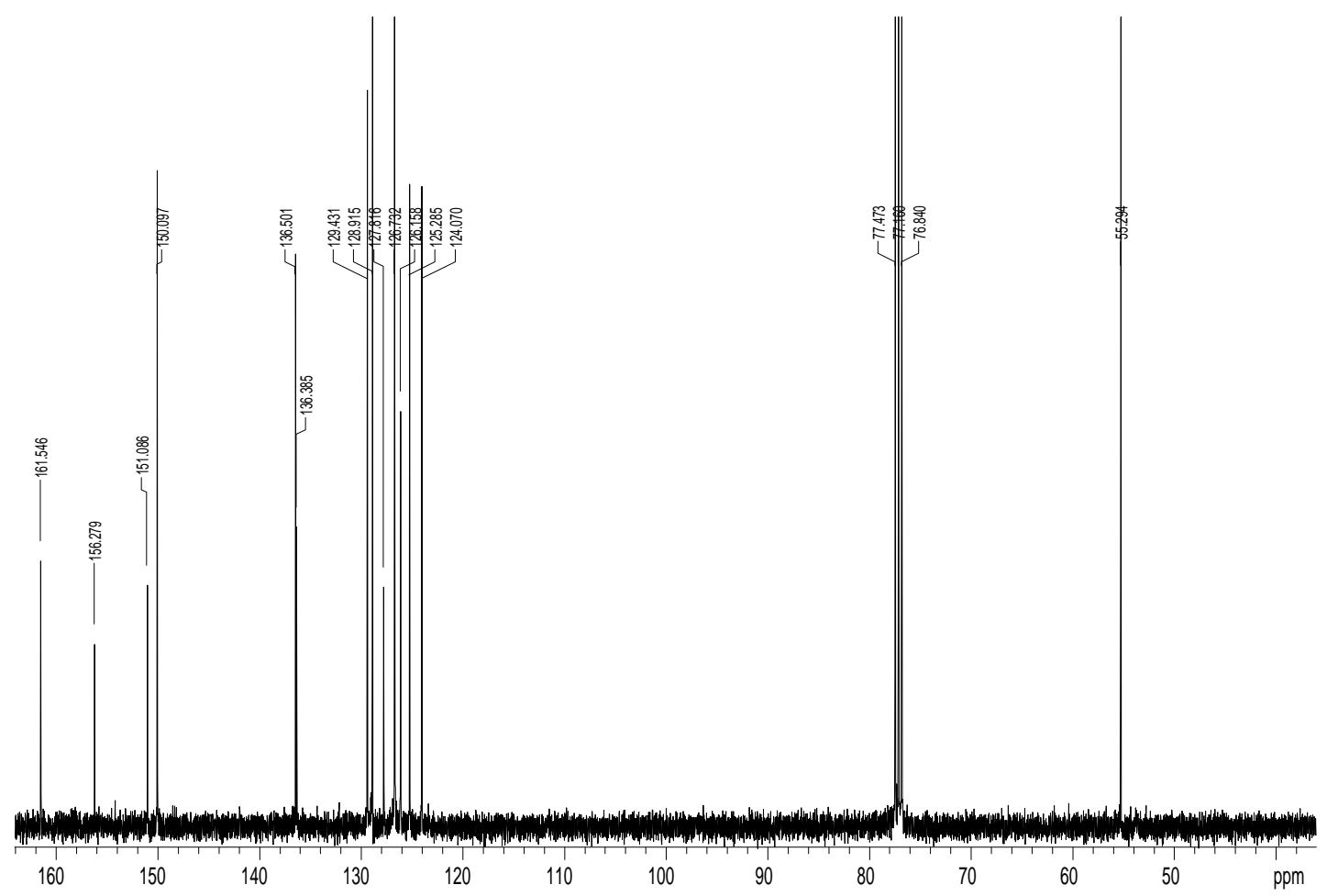


3-Methoxy-6-phenyl-4-(pyrimidin-5-yl)pyridazine (22)

${ }^{1} \mathrm{H} \mathrm{NMR}\left(400 \mathrm{MHz}, \mathrm{CDCl}_{3}\right)$

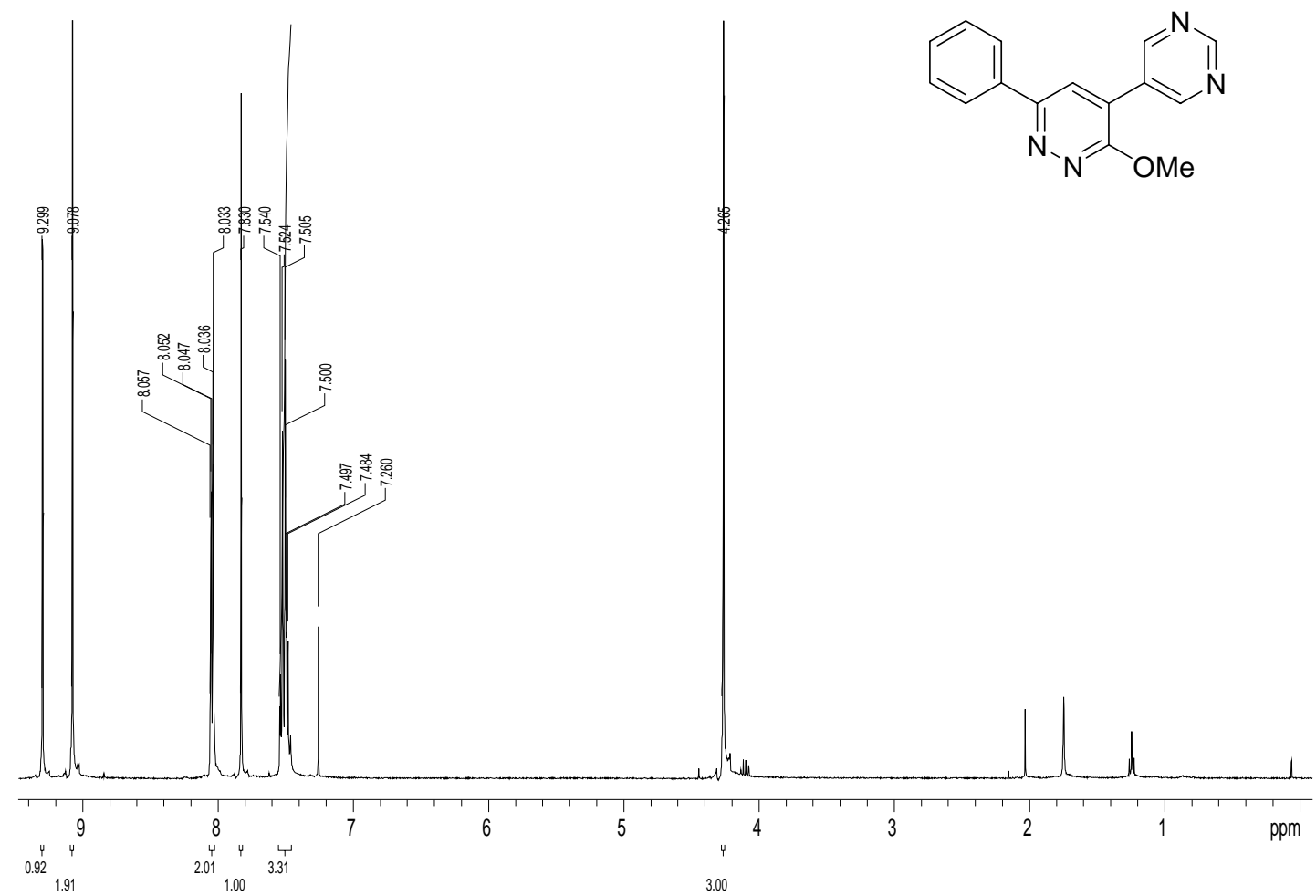

${ }^{13} \mathrm{C}$ NMR $\left(100 \mathrm{MHz}, \mathrm{CDCl}_{3}\right)$

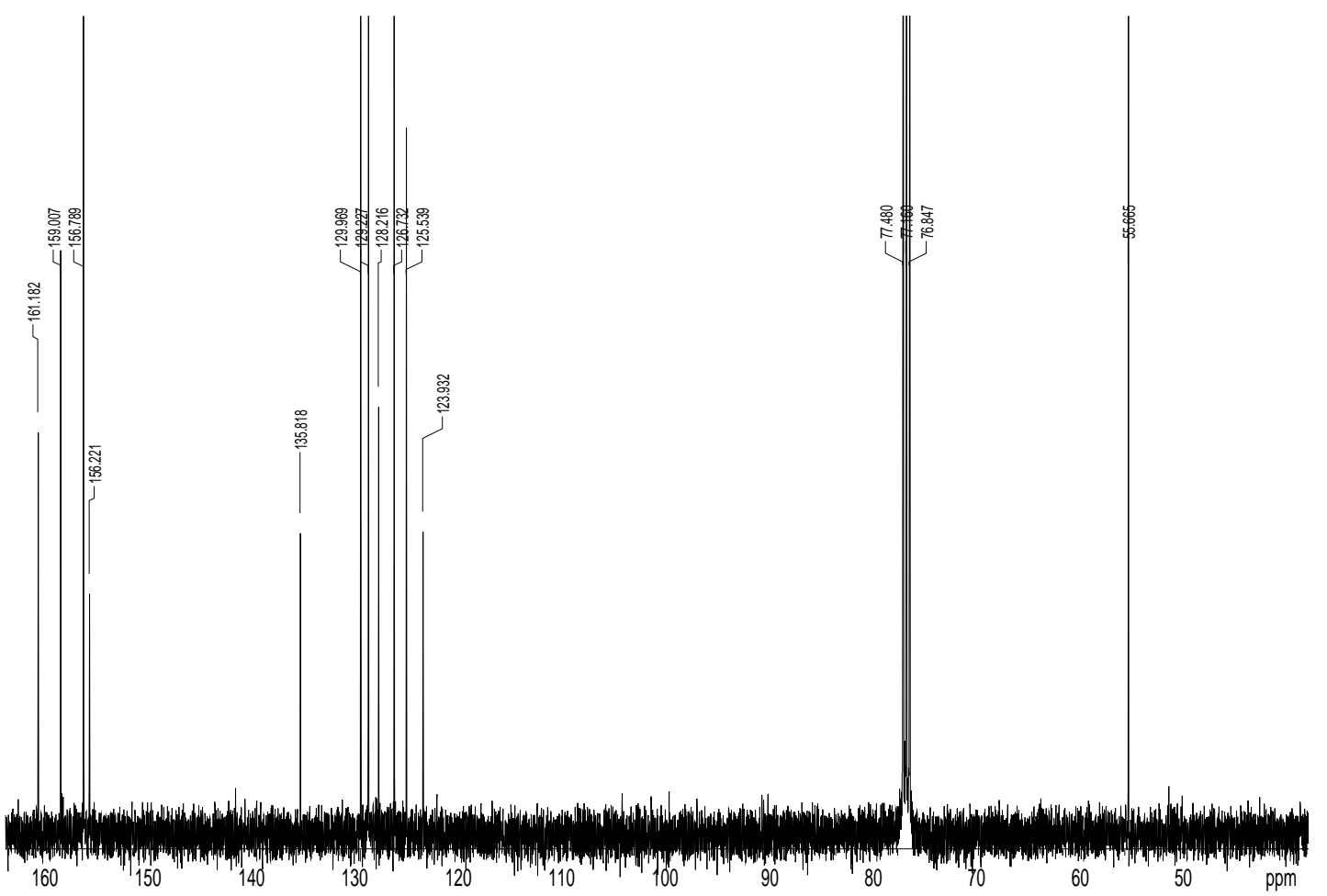


2-Amino-3-nitro-5-(3-methoxy-6-phenylpyridazin-4-yl)-pyridine (23)

${ }^{1} \mathrm{H}$ NMR (400 MHz, DMSO-d 6 )

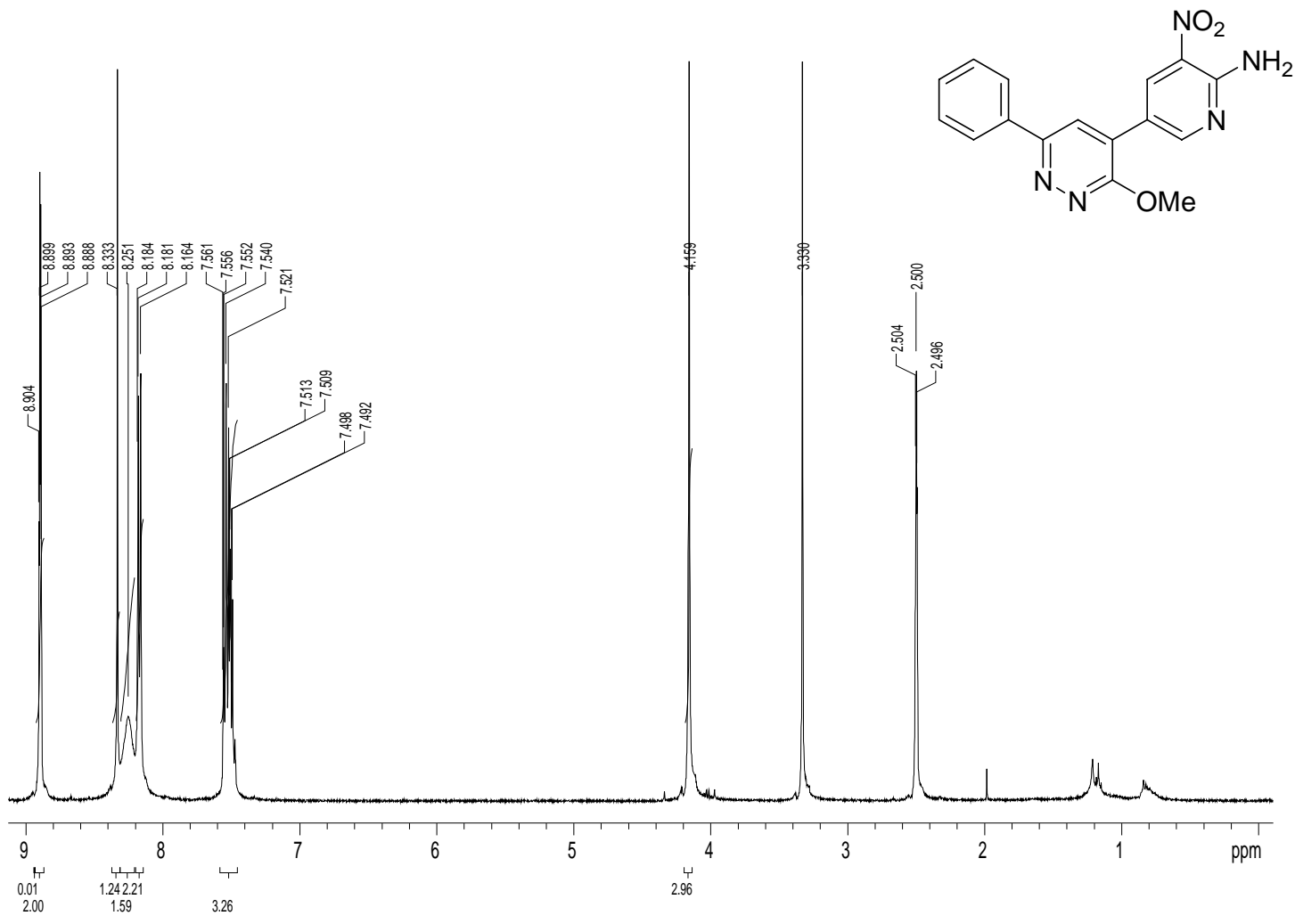

${ }^{13} \mathrm{C}$ NMR (100 MHz, DMSO-d 6 )

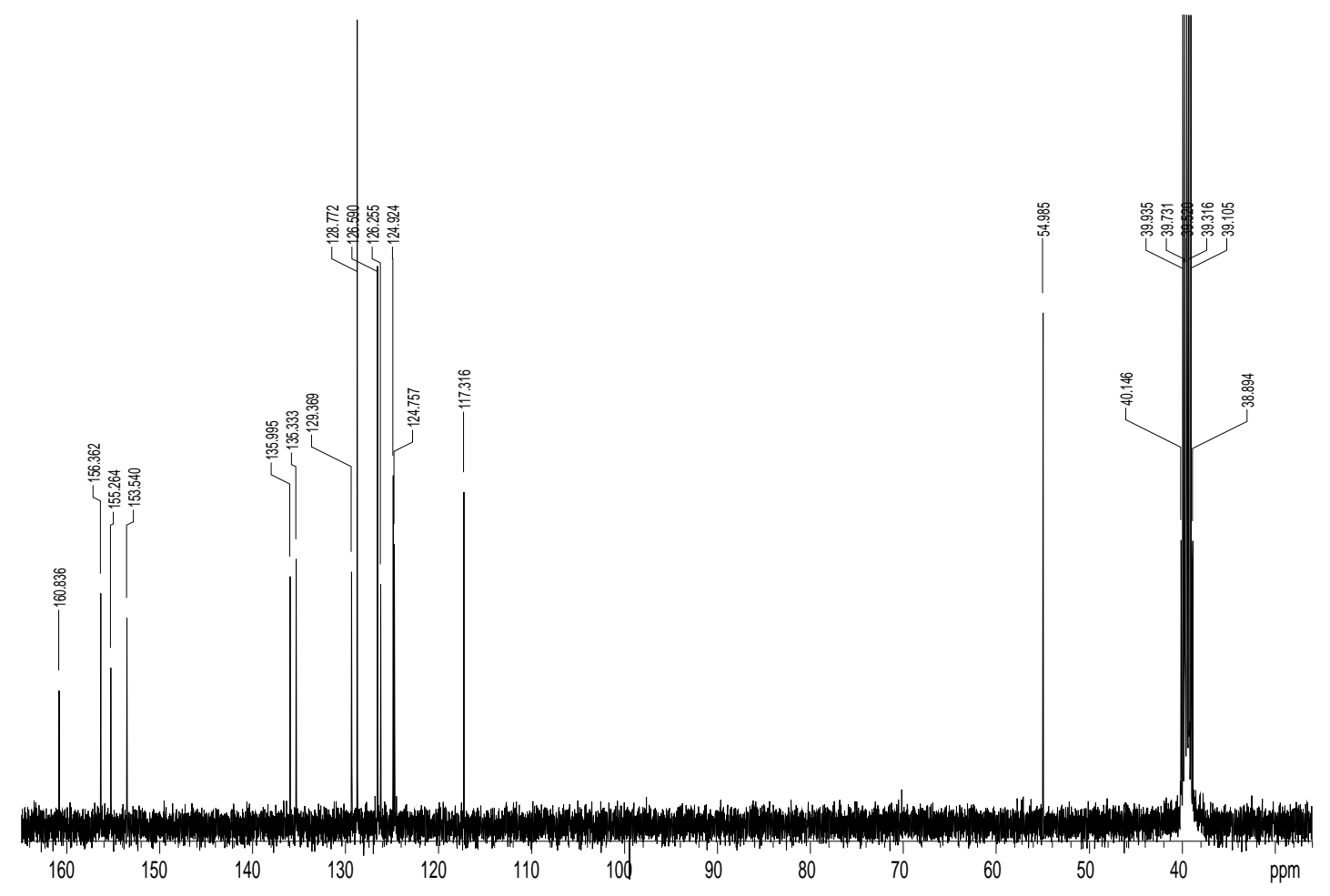


3-Methoxy-6-(4-methoxyphenyl)-4-(pyridin-2-yl)pyridazine (24)

${ }^{1} \mathrm{H}$ NMR (400 MHz, $\mathrm{CDCl}_{3}$ )
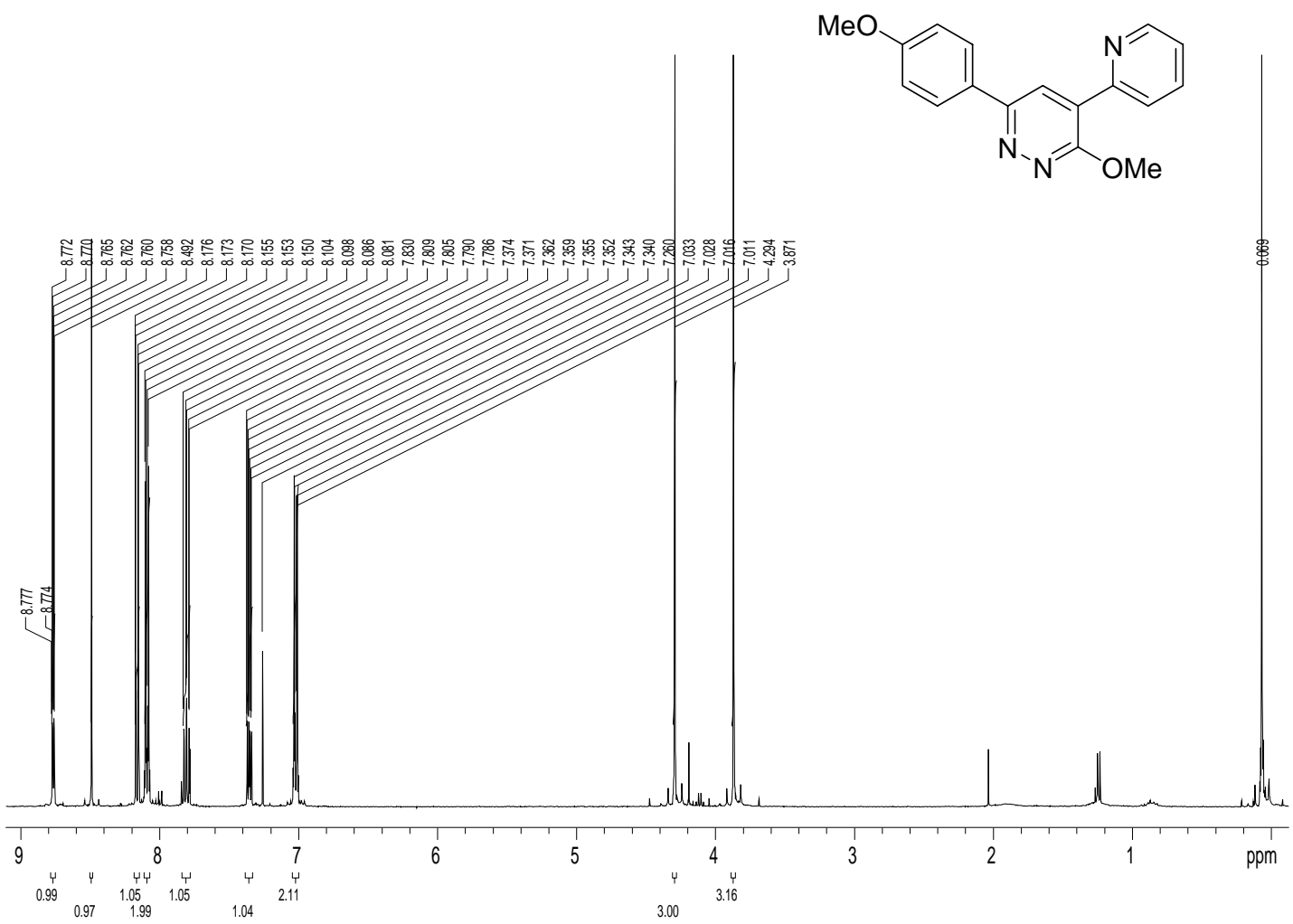

${ }^{13} \mathrm{C}$ NMR (100 MHz, $\left.\mathrm{CDCl}_{3}\right)$

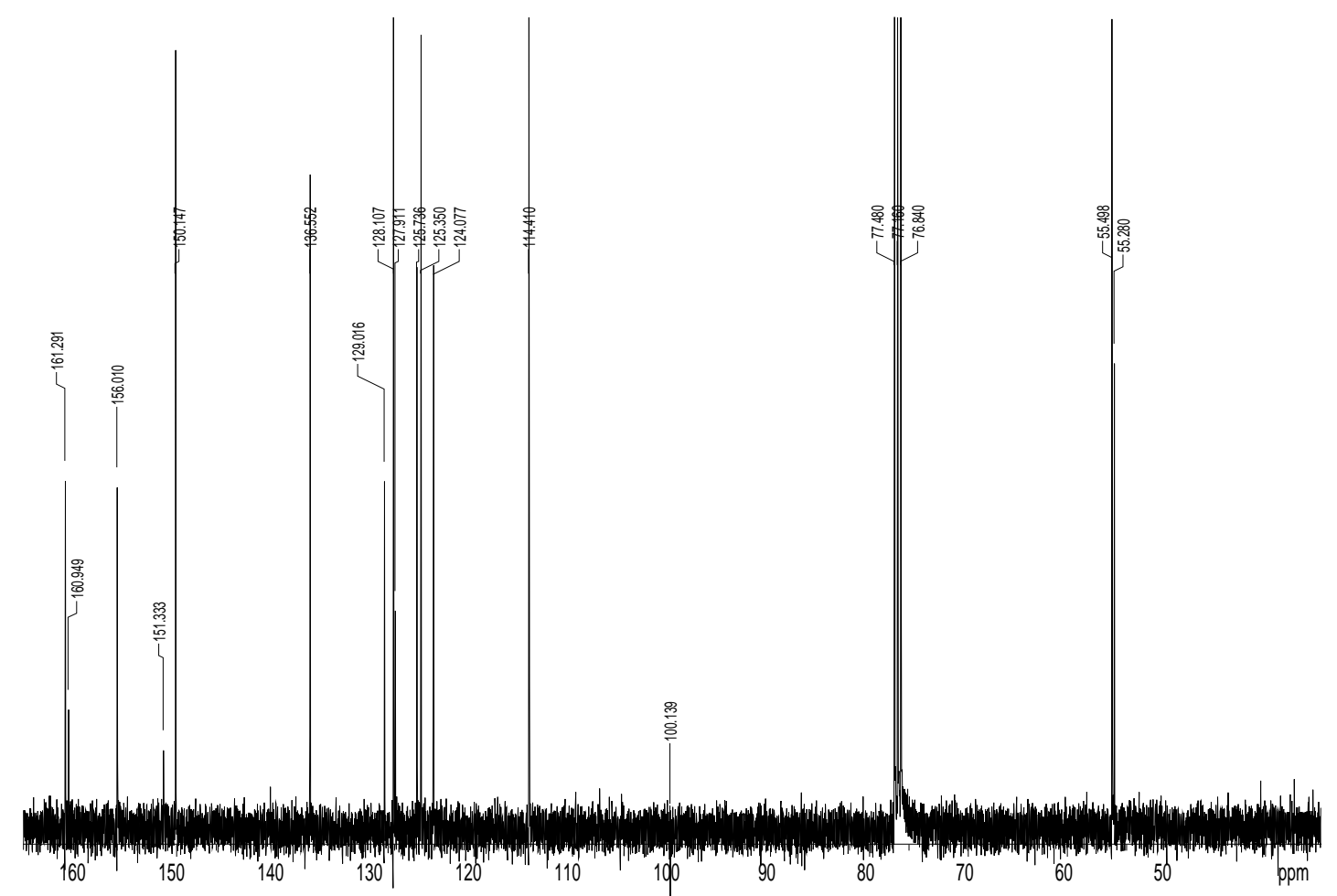


3-Methoxy-6-(4-methoxyphenyl)-4-(pyrimidin-5-yl)pyridazine (25)

${ }^{1} \mathrm{H}$ NMR (400 MHz, $\mathrm{CDCl}_{3}$ )

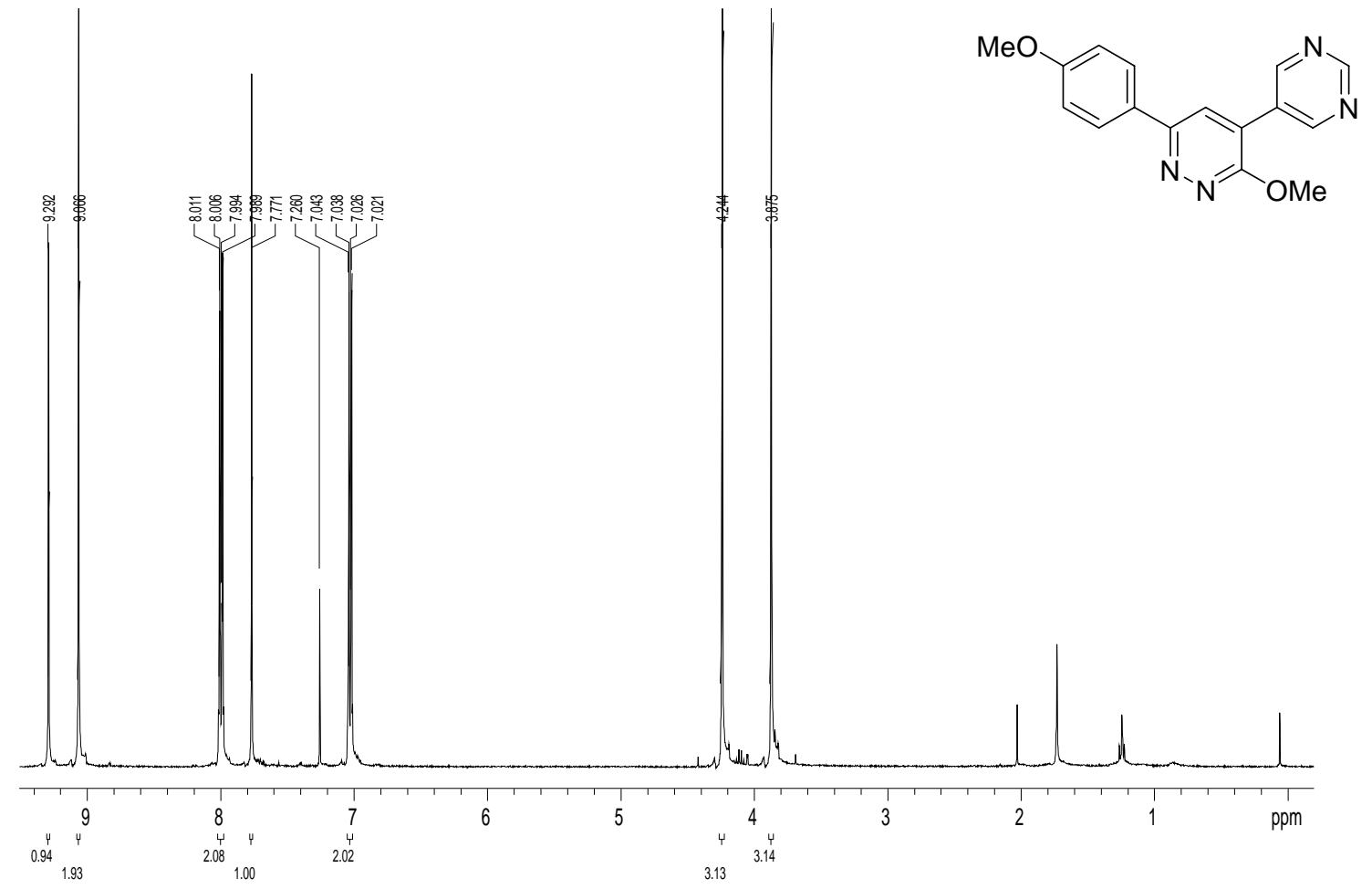

${ }^{13} \mathrm{C}$ NMR $\left(100 \mathrm{MHz}, \mathrm{CDCl}_{3}\right)$

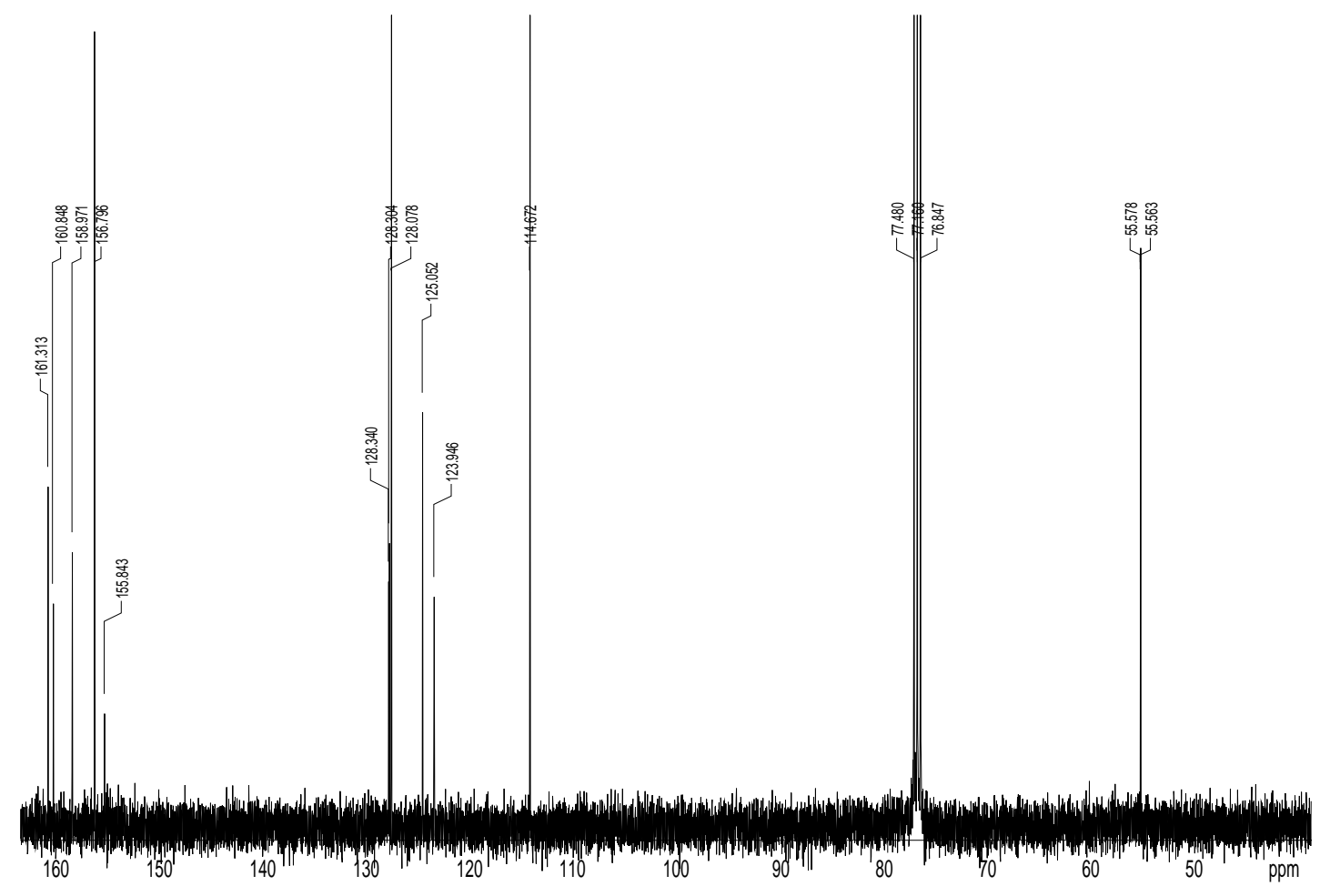


5-(3-Methoxy-6-(4-methoxyphenyl)pyridazin-4-yl)-3-nitropyridin-2-amine (26)

${ }^{1} \mathrm{H}$ NMR (400 MHz, DMSO-d )

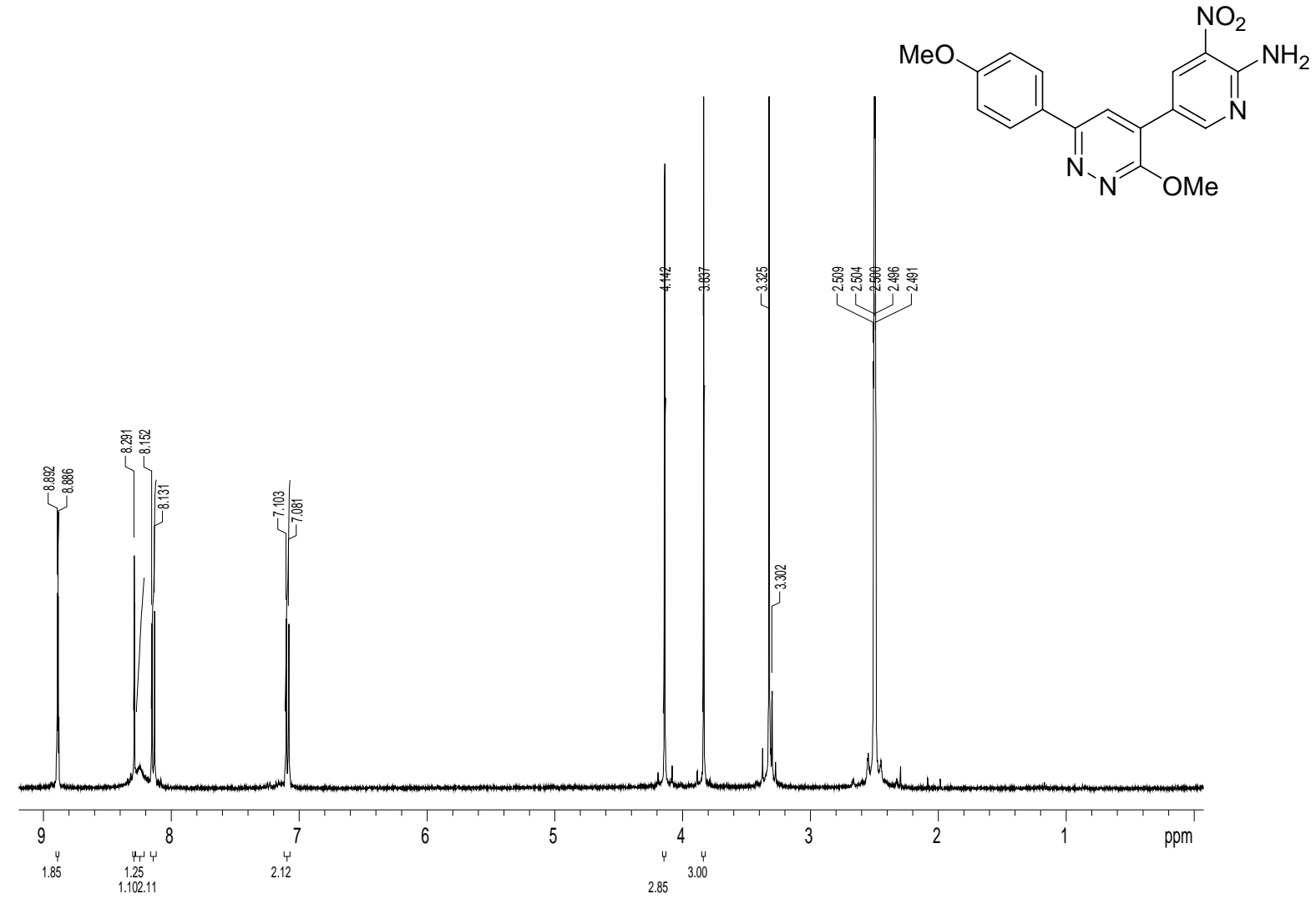

${ }^{13} \mathrm{C}$ NMR (125 MHz, DMSO-d 6 )

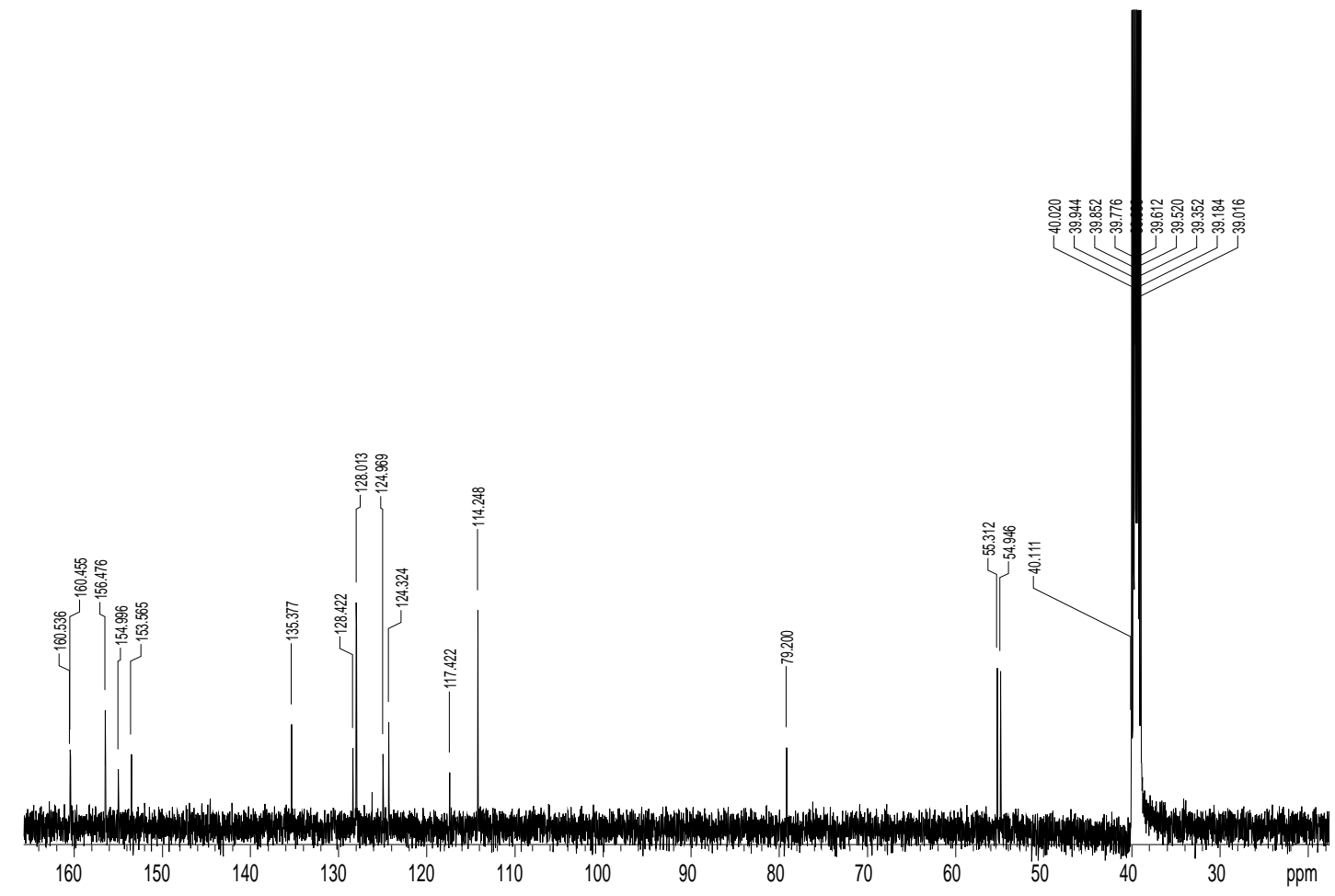


3-Methoxy-6-(2-methoxypyridin-5-yl)-4-(pyridin-2-yl)pyridazine (27)

${ }^{1} \mathrm{H}$ NMR (400 MHz, $\mathrm{CDCl}_{3}$ )

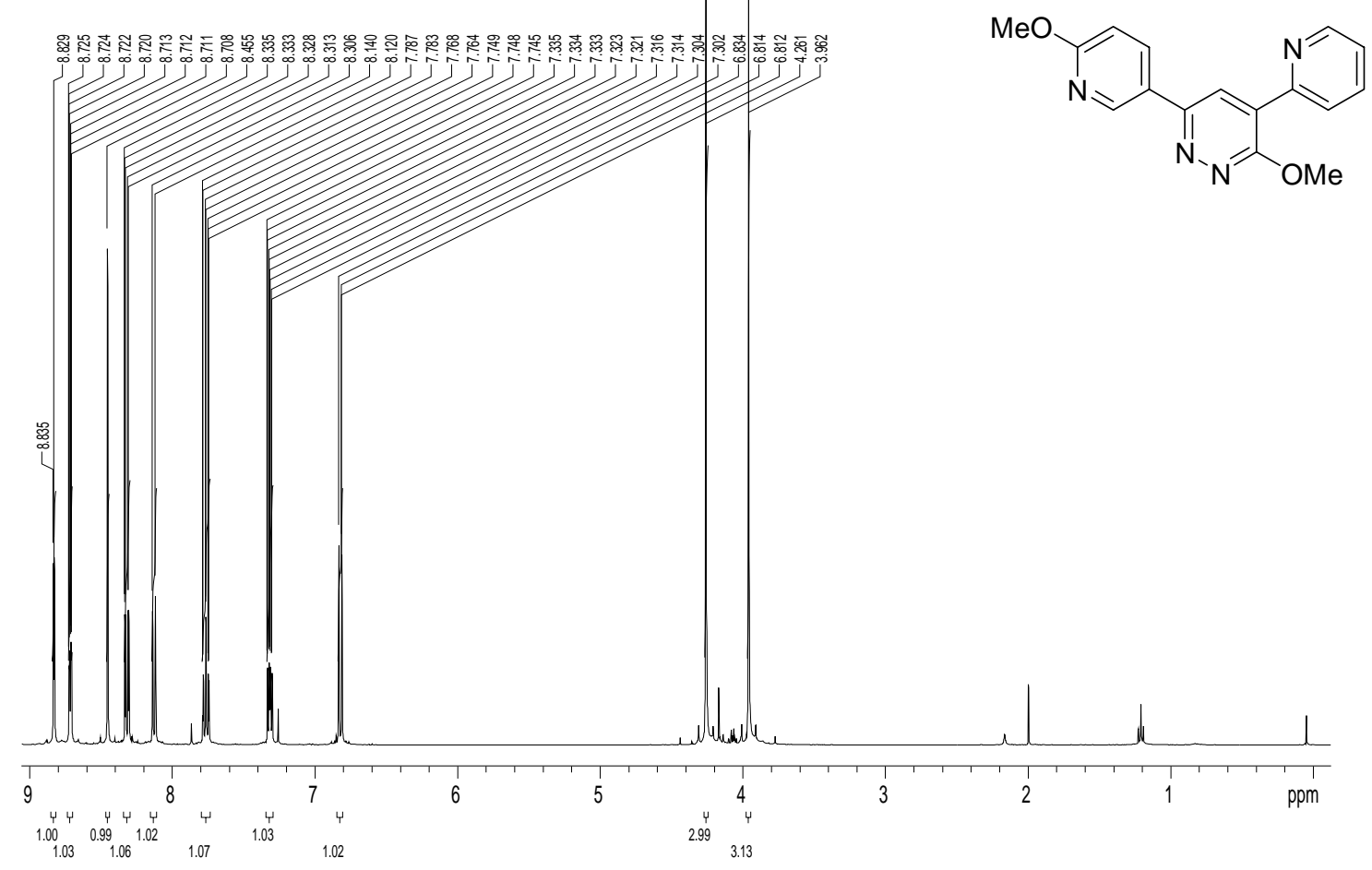

${ }^{13} \mathrm{C}$ NMR (100 MHz, $\left.\mathrm{CDCl}_{3}\right)$

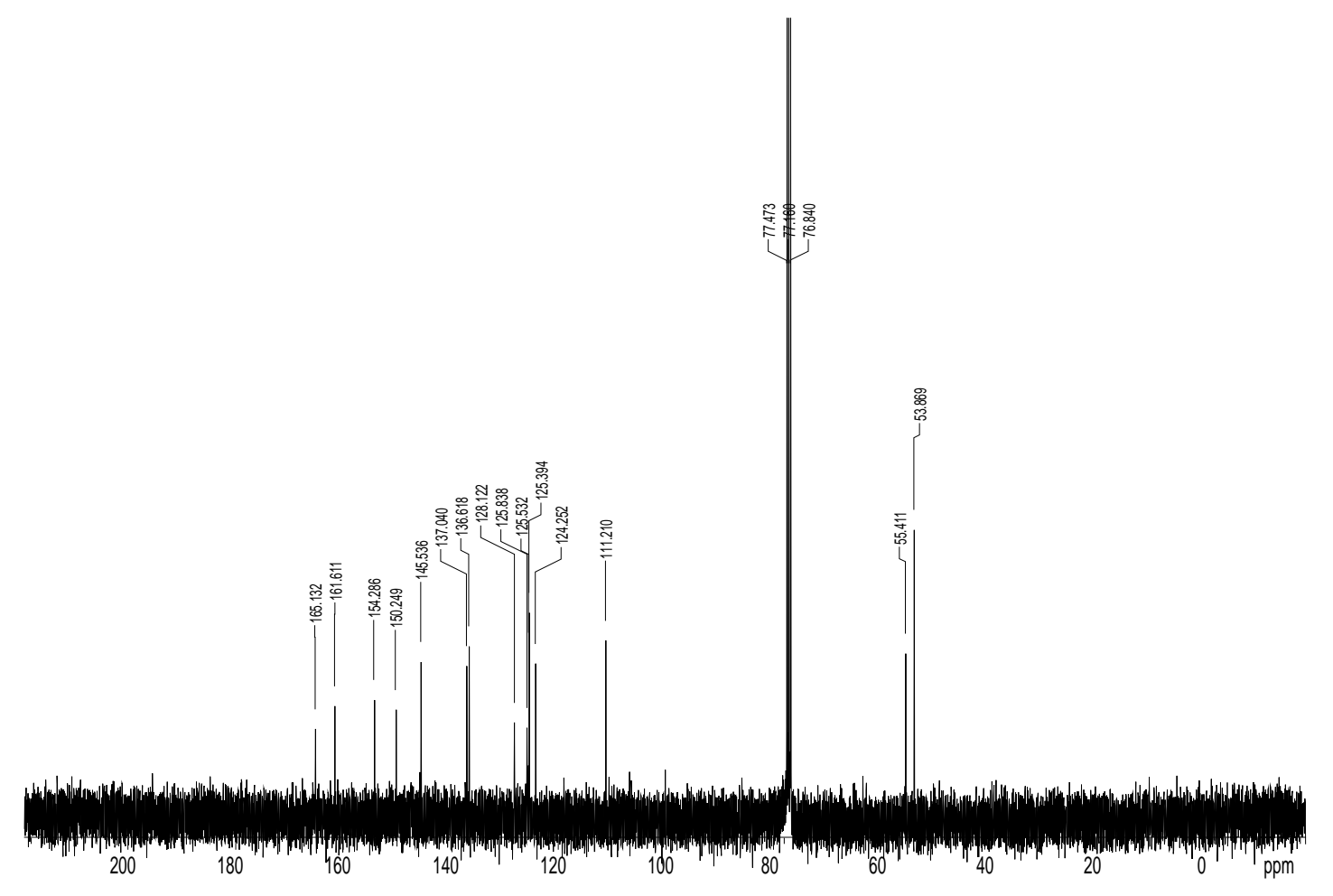


3-Methoxy-6-(2-methoxypyridin-5-yl)-4-(pyrimidin-5-yl)pyridazine (28)

${ }^{1} \mathrm{H}$ NMR (400 MHz, $\mathrm{CDCl}_{3}$ )

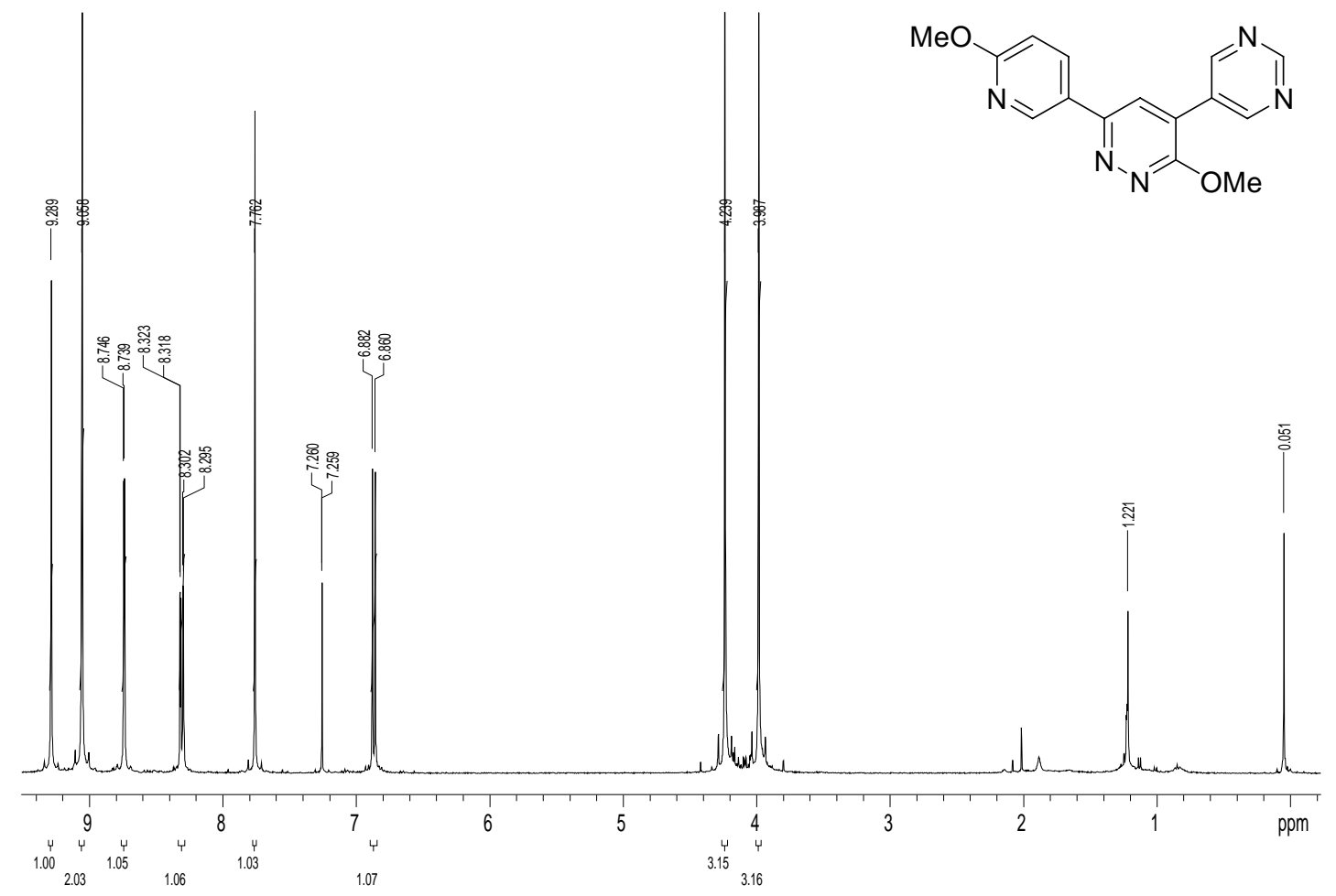

${ }^{13} \mathrm{C}$ NMR (125 MHz, $\mathrm{CDCl}_{3}$ )

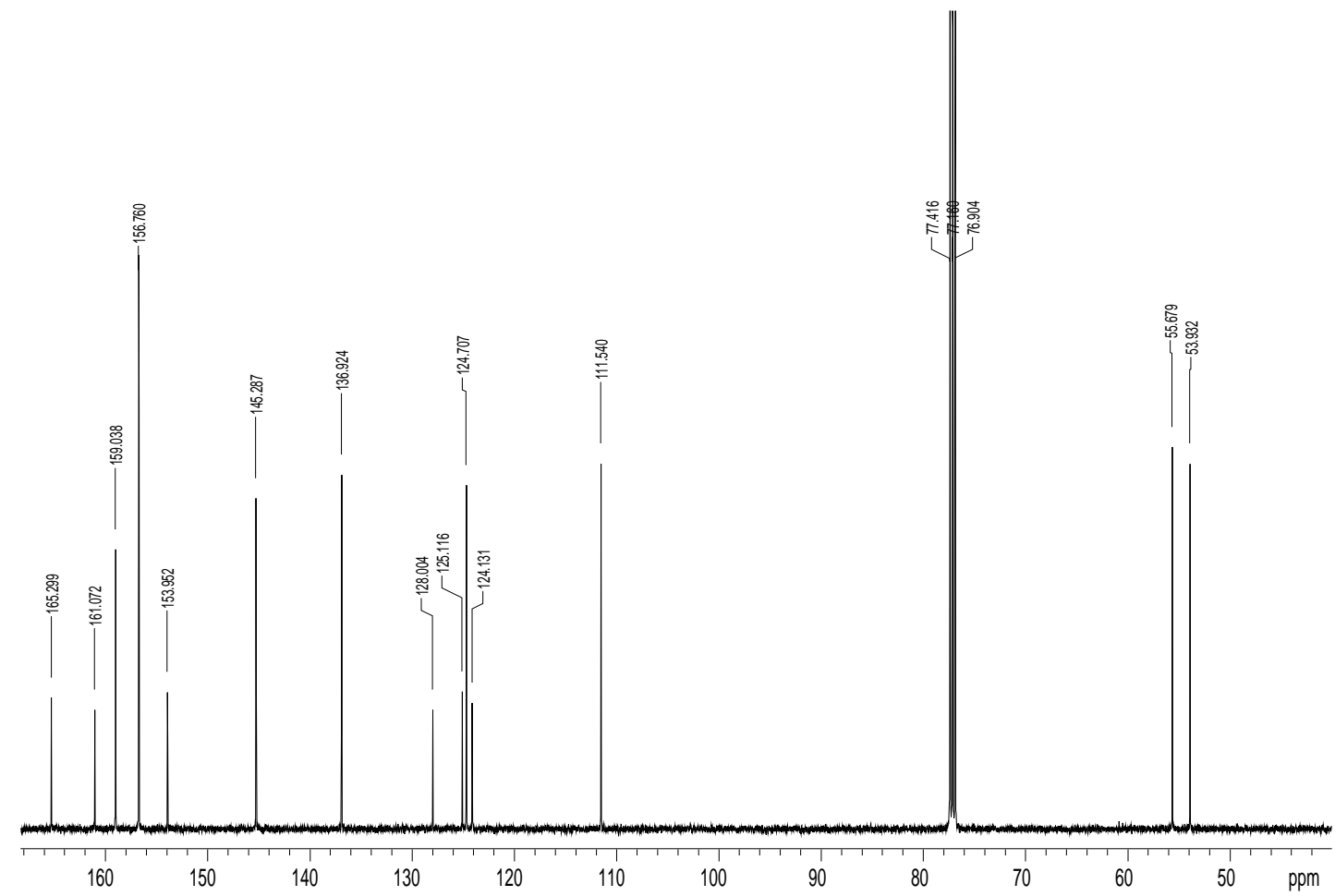


5-(3-Methoxy-6-(2-methoxypyridin-5-yl)-pyridazin-4-yl)-3-nitropyridin-2-amine (29) ${ }^{1} \mathrm{H}$ NMR (400 MHz, DMSO-d )

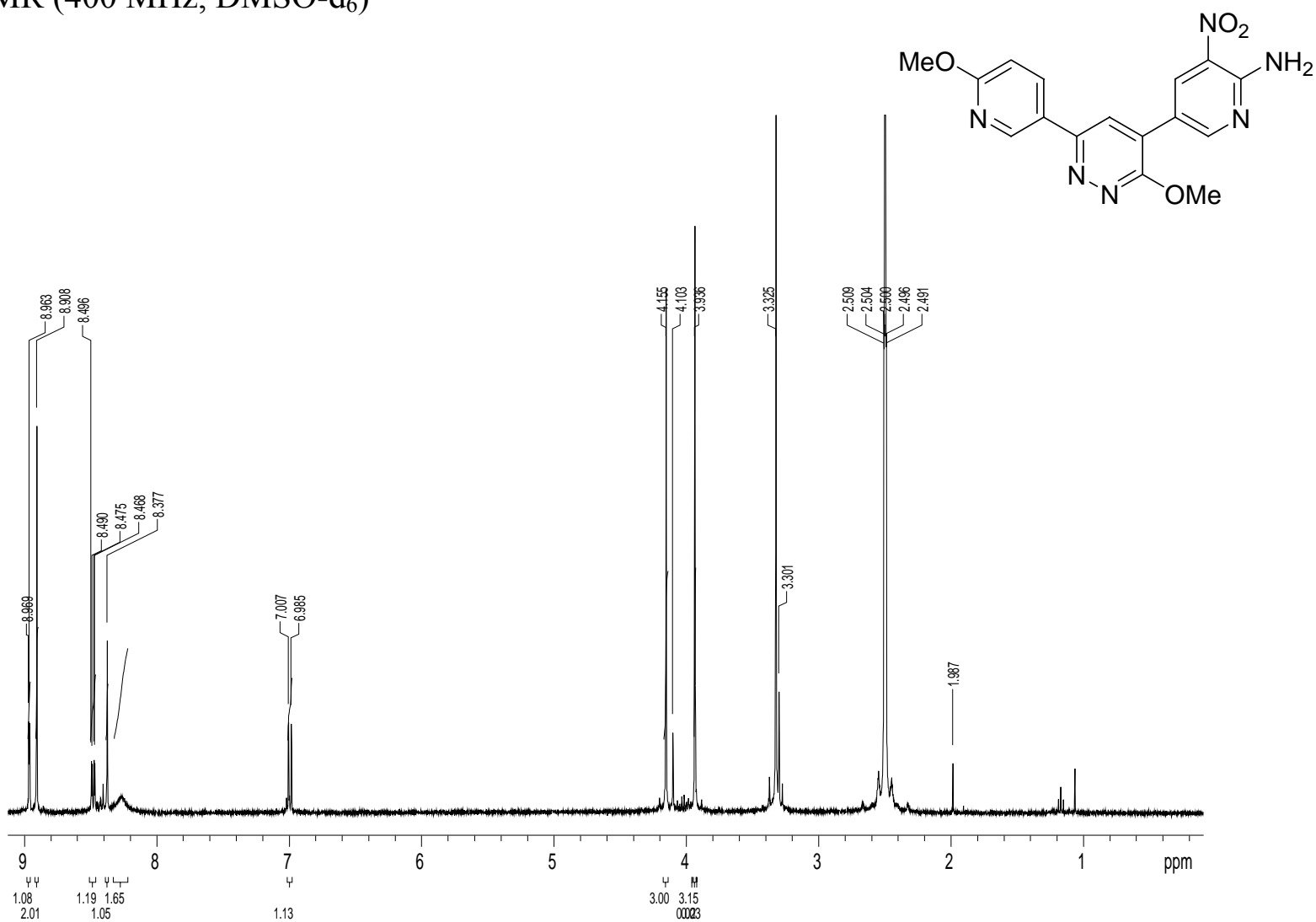

${ }^{13} \mathrm{C}$ NMR (125 MHz, DMSO-d 6 )

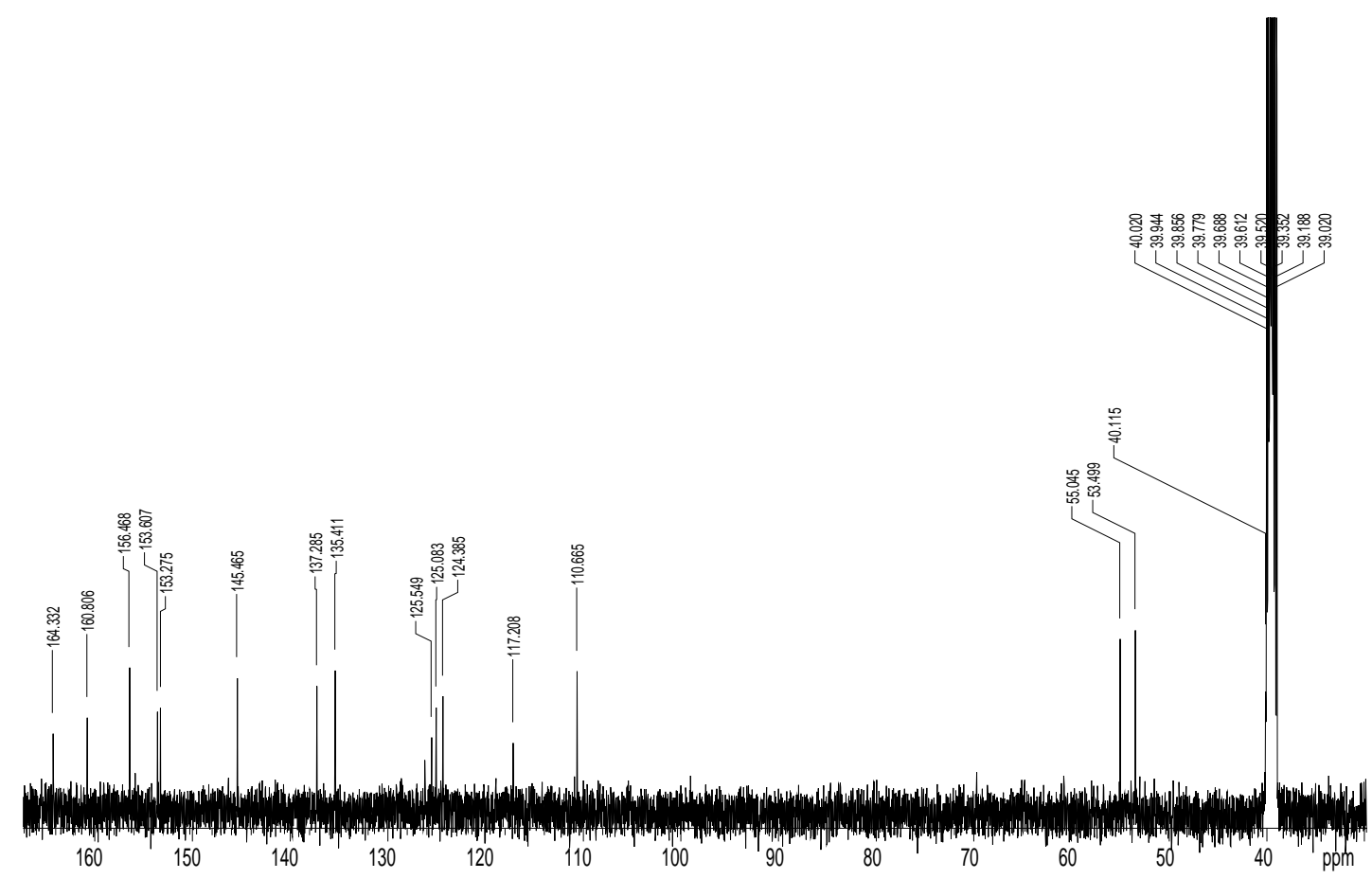


3-Methoxy-6-(2-fluoropyridin-5-yl)-4-(pyridin-2-yl)pyridazine (30)

${ }^{1} \mathrm{H}$ NMR (400 MHz, $\mathrm{CDCl}_{3}$ )
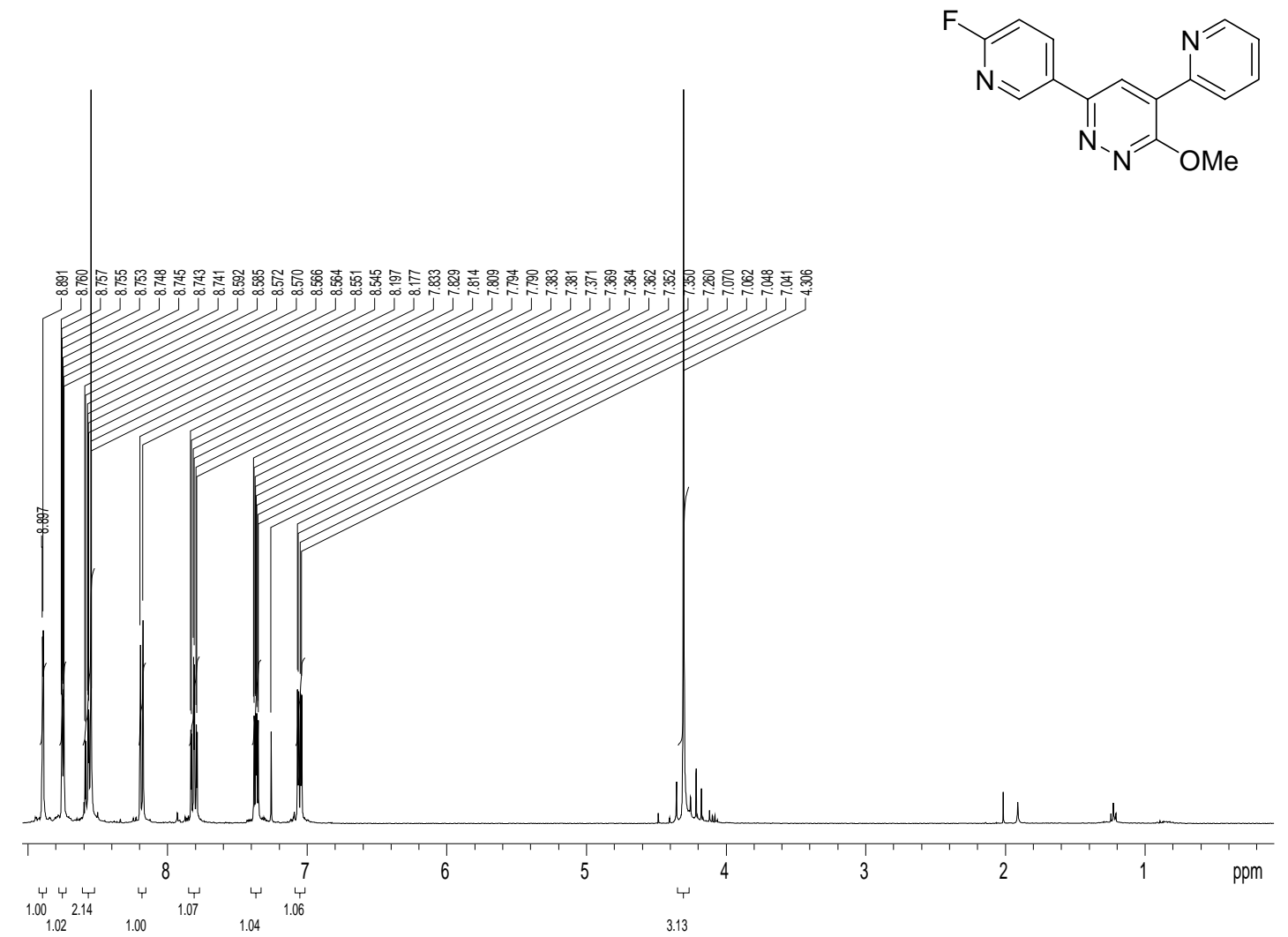

${ }^{13} \mathrm{C}$ NMR (100 MHz, $\mathrm{CDCl}_{3}$ )

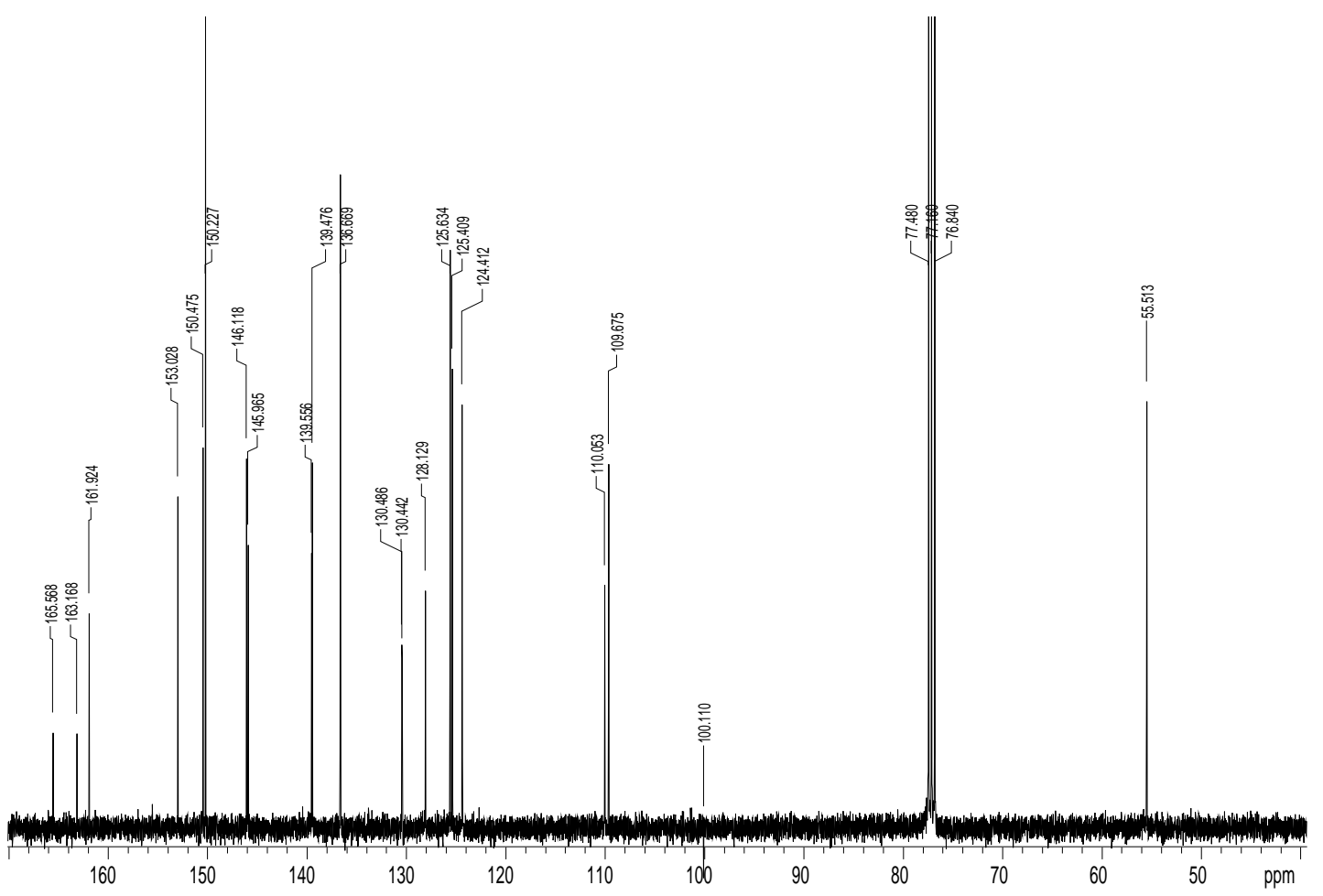


3-Methoxy-6-(2-fluoropyridin-5-yl)-4-(pyrimidin-5-yl)pyridazine (31)

${ }^{1} \mathrm{H}$ NMR (400 MHz, $\mathrm{CDCl}_{3}$ )

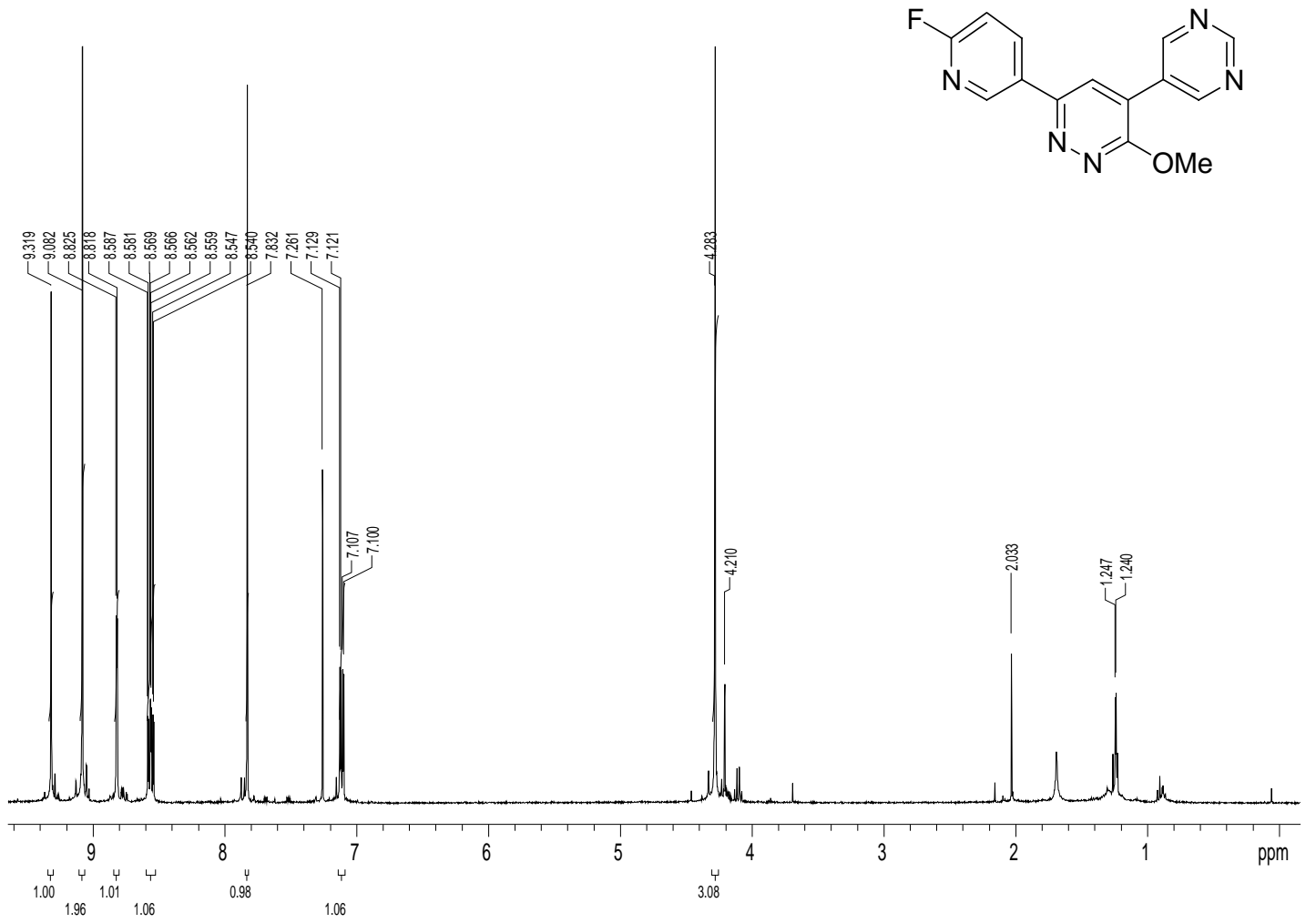

${ }^{13} \mathrm{C}$ NMR $\left(100 \mathrm{MHz}, \mathrm{CDCl}_{3}\right)$

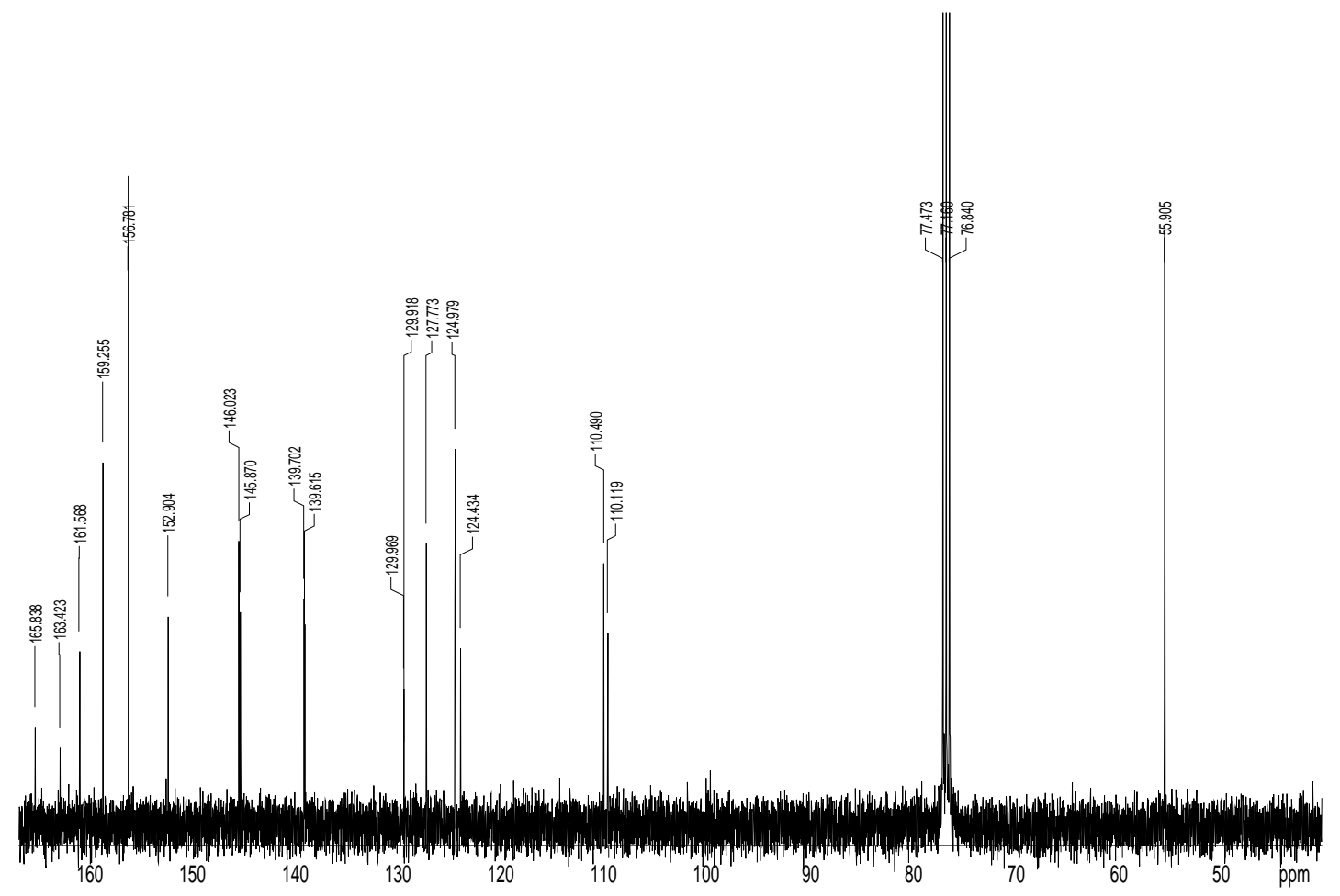


5-(6-(2-Fluoropyridin-5-yl)-3-methoxypyridazin-4-yl)-3-nitropyridin-2-amine (32)

${ }^{1} \mathrm{H}$ NMR (400 MHz, DMSO-d )

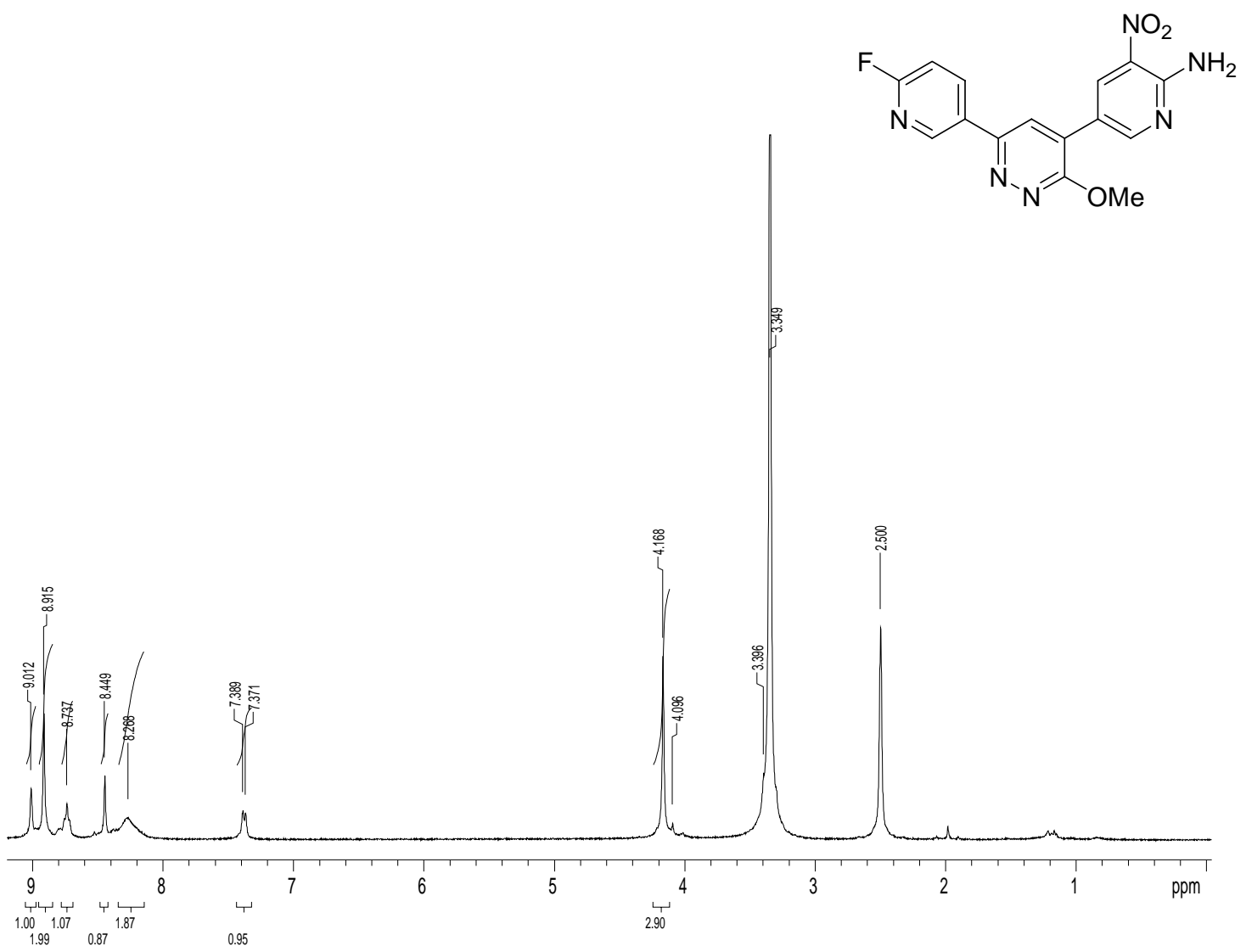

${ }^{13} \mathrm{C}$ NMR (125 MHz, DMSO-d 6 )

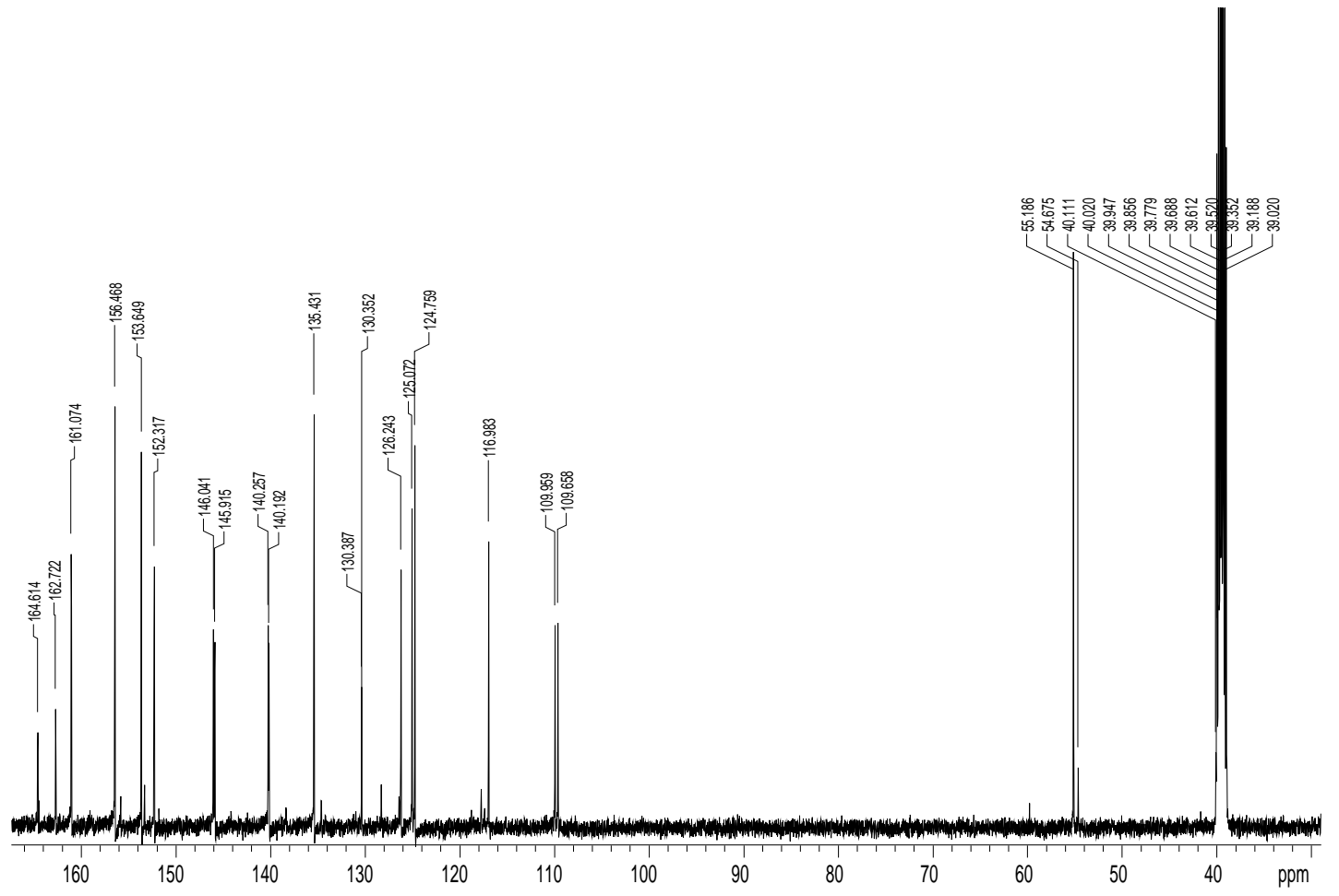


Potassium 3-methoxy-6-phenyl-4-pyridazinyltrifluoroborate salt (34)

${ }^{1} \mathrm{H}$ NMR (400 MHz, DMSO-d 6 )

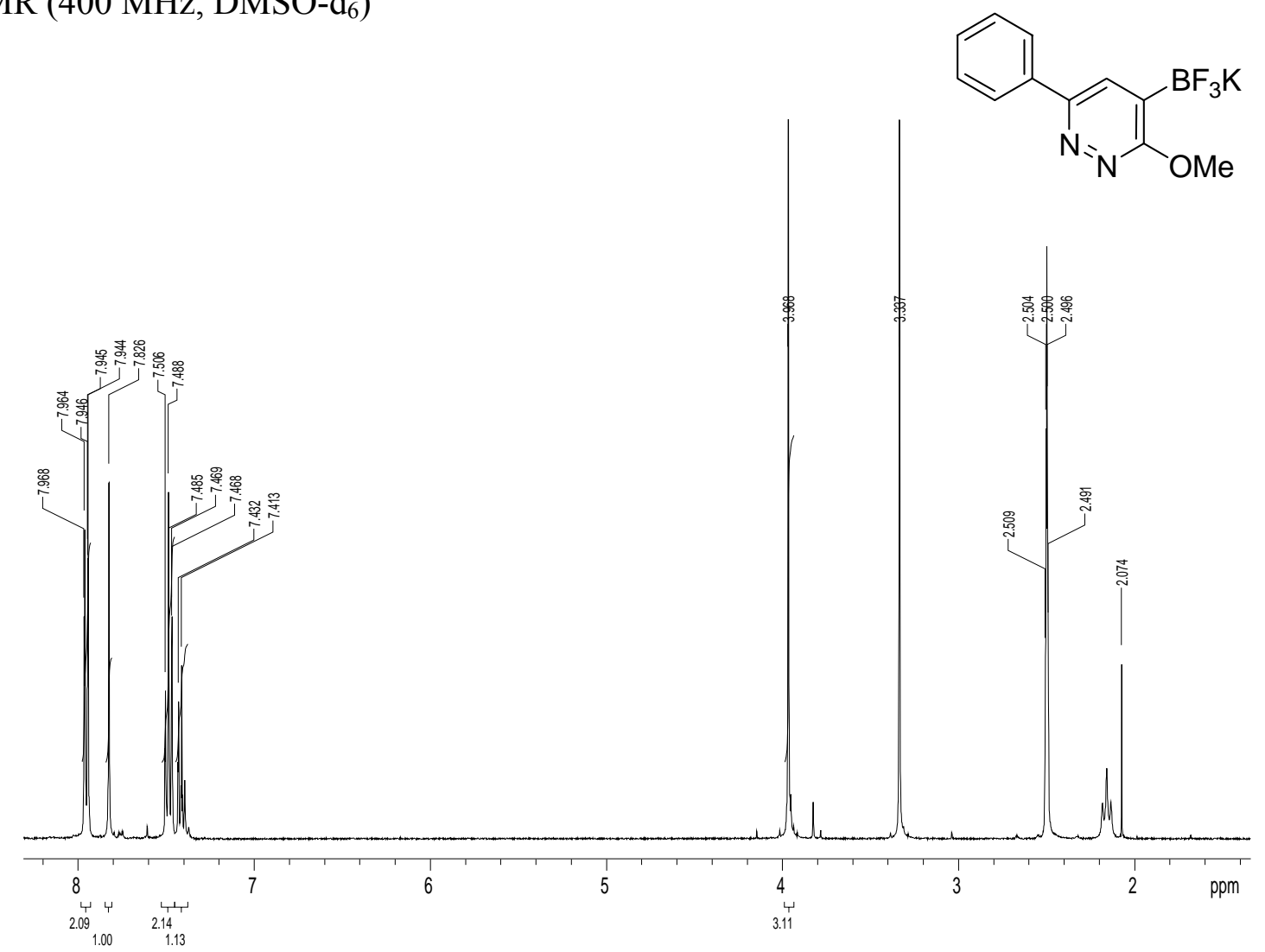

${ }^{13} \mathrm{C}$ NMR (100 MHz, DMSO-d 6 )

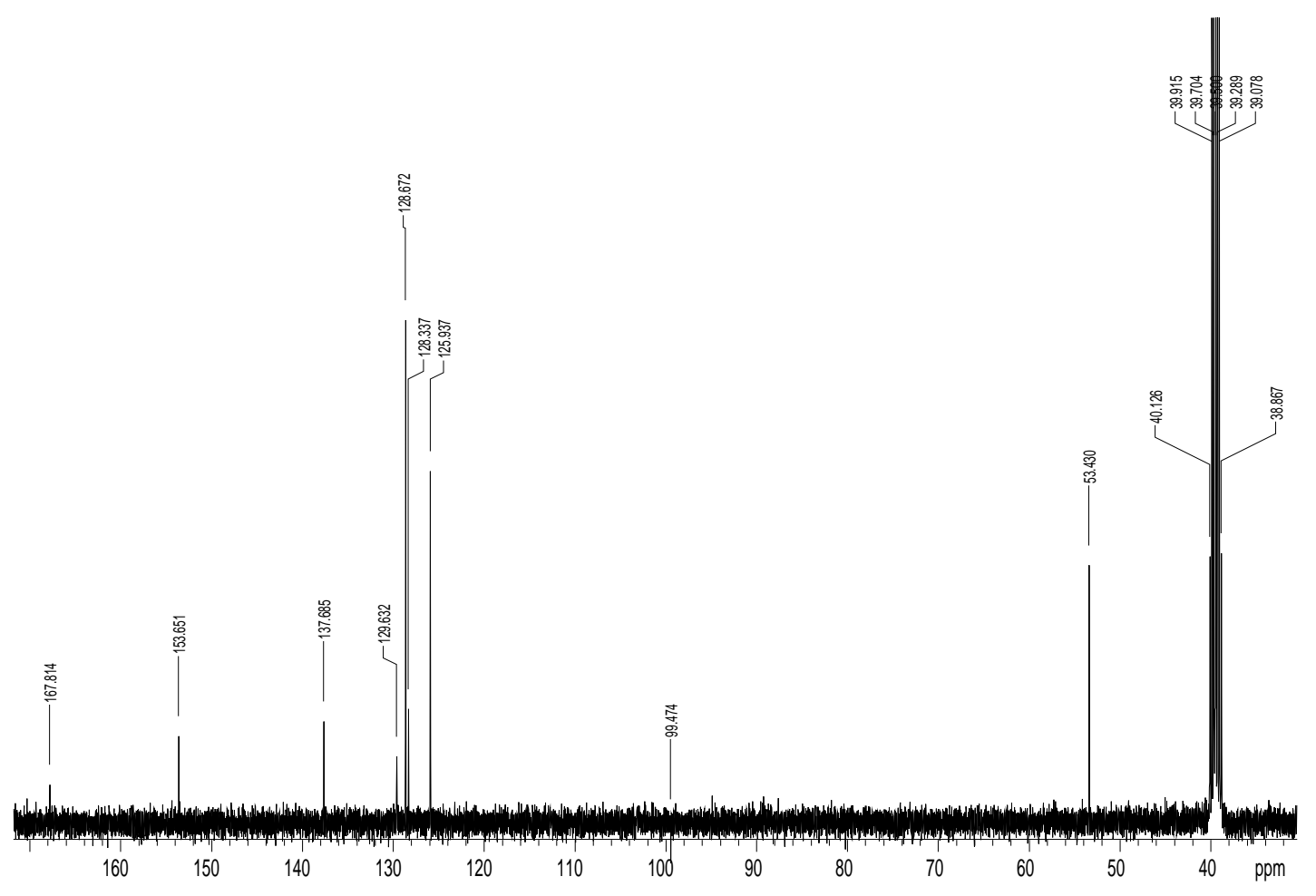


6-(6-Chloropyridin-3-yl)pyridazin-3(2H)-one (35)

${ }^{1} \mathrm{H}$ NMR (400 MHz, DMSO-d )

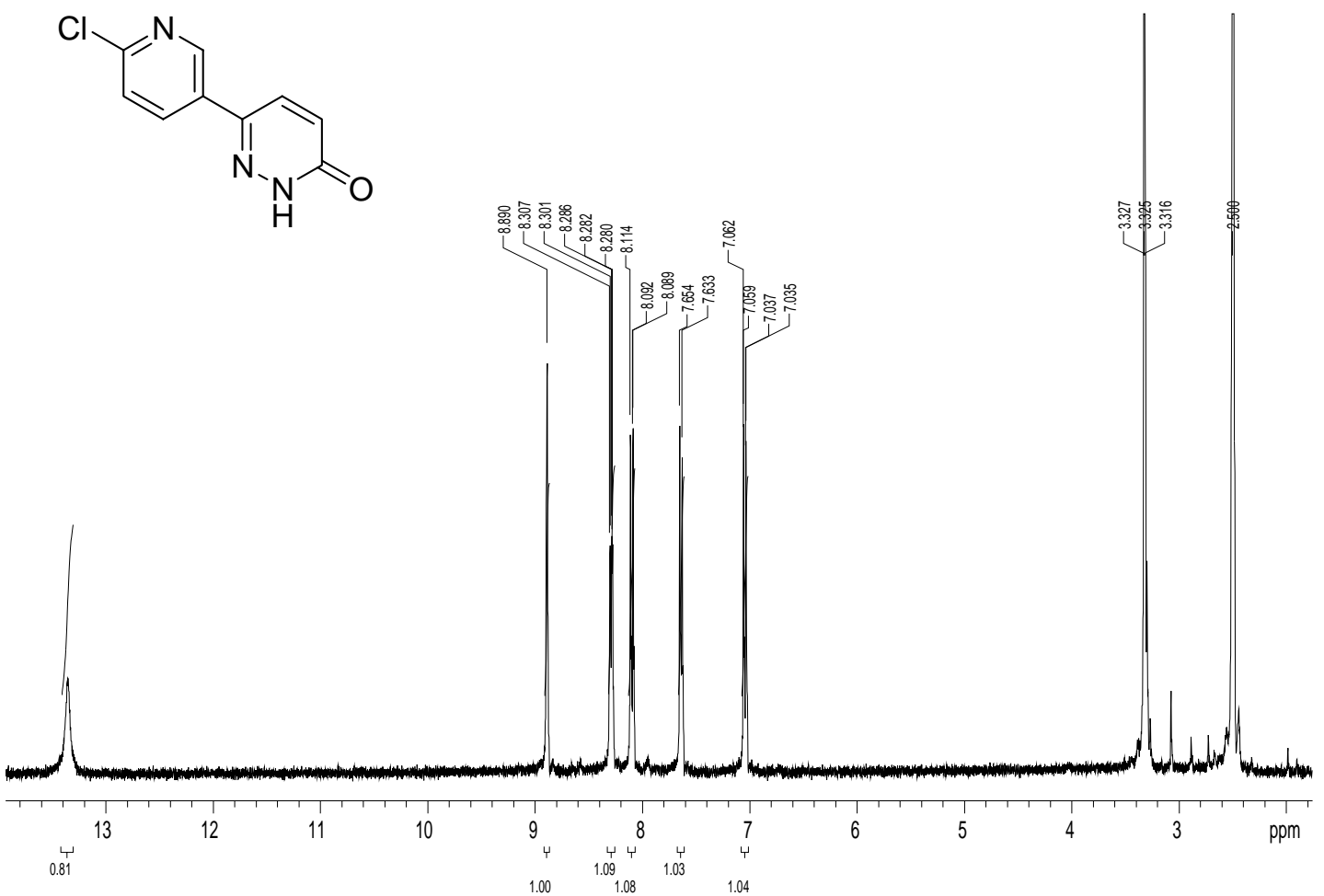

${ }^{13} \mathrm{C}$ NMR (100 MHz, DMSO-d 6 )
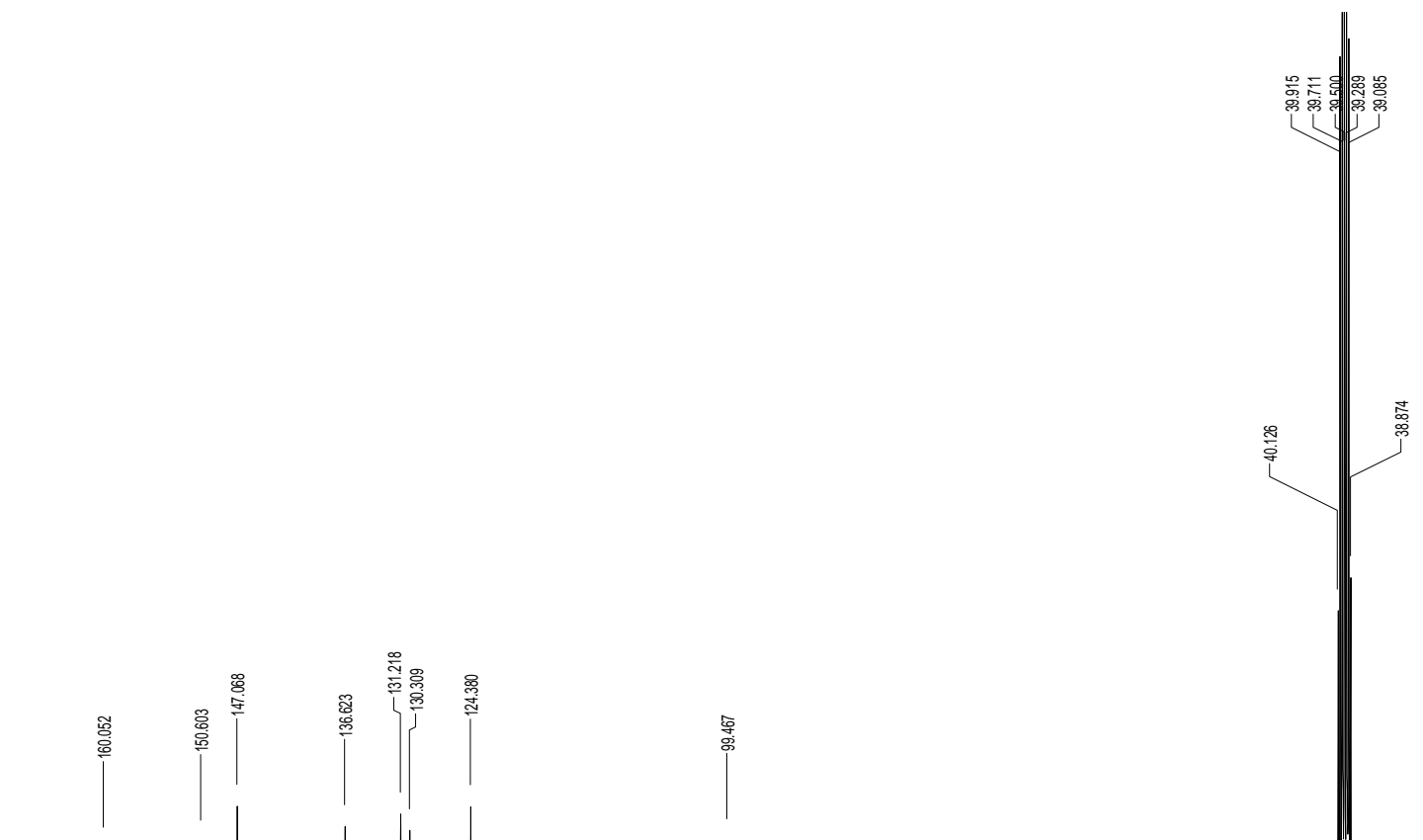

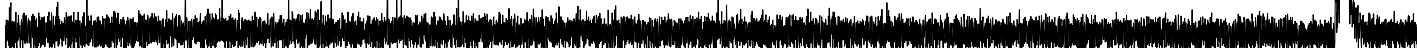


6-Phenyl-4-(pyridin-2-yl)pyridazin-3(2H)-one (36)

${ }^{1} \mathrm{H}$ NMR (400 MHz, DMSO-d )<smiles>O=c1[nH]nc(-c2ccccc2)cc1-c1ccccn1</smiles>

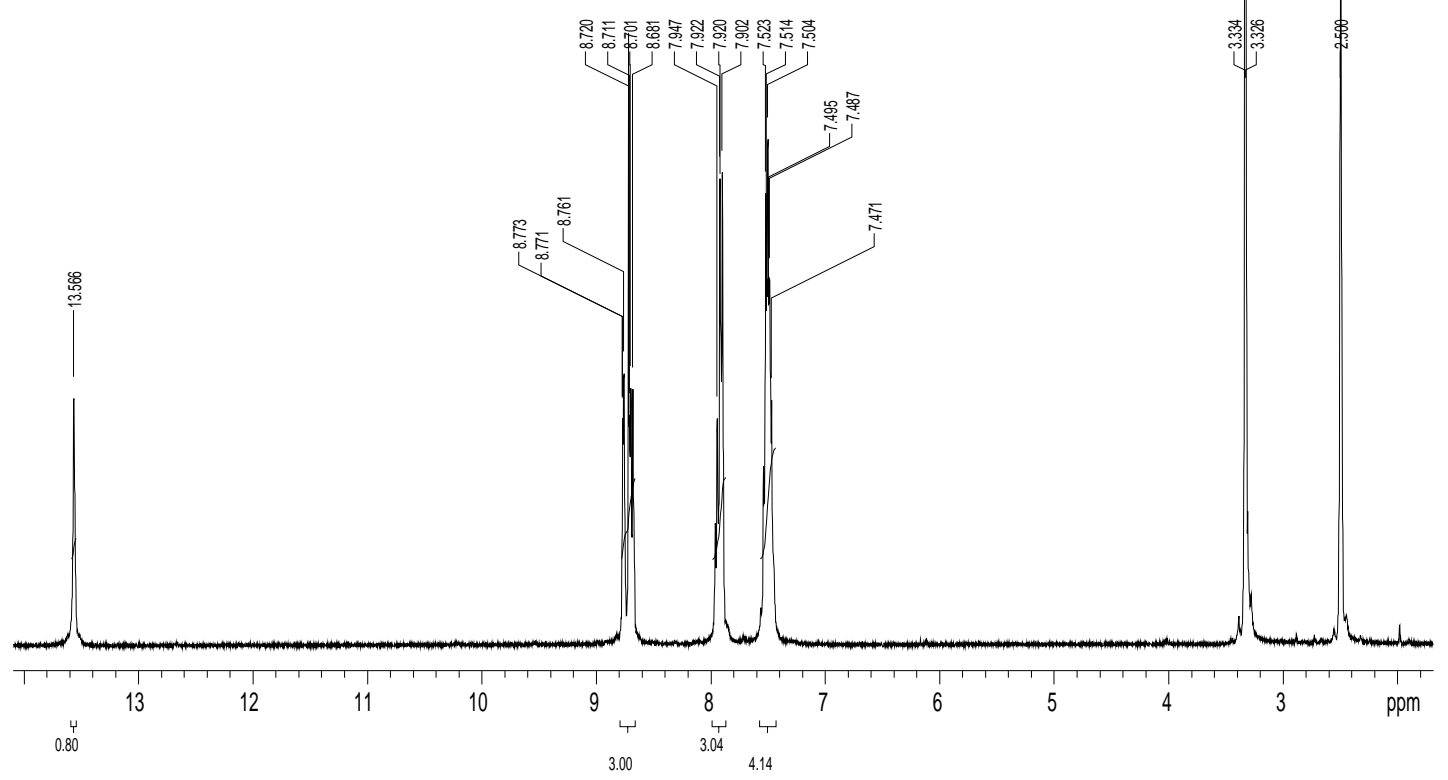

${ }^{13} \mathrm{C}$ NMR (100 MHz, DMSO-d 6 )

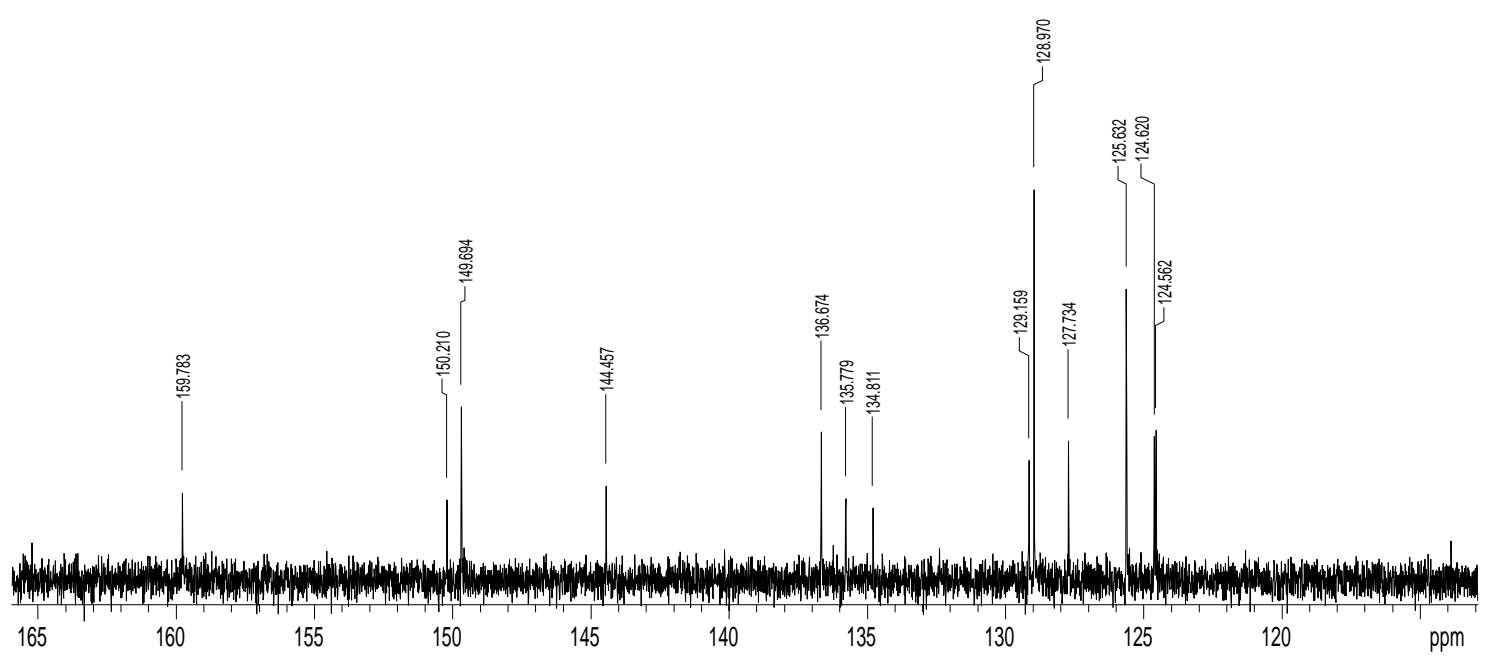


6-Phenyl-4-(pyrimidin-5-yl)pyridazin-3(2H)-one (37)

${ }^{1} \mathrm{H}$ NMR (400 MHz, DMSO-d ${ }_{6}$ )

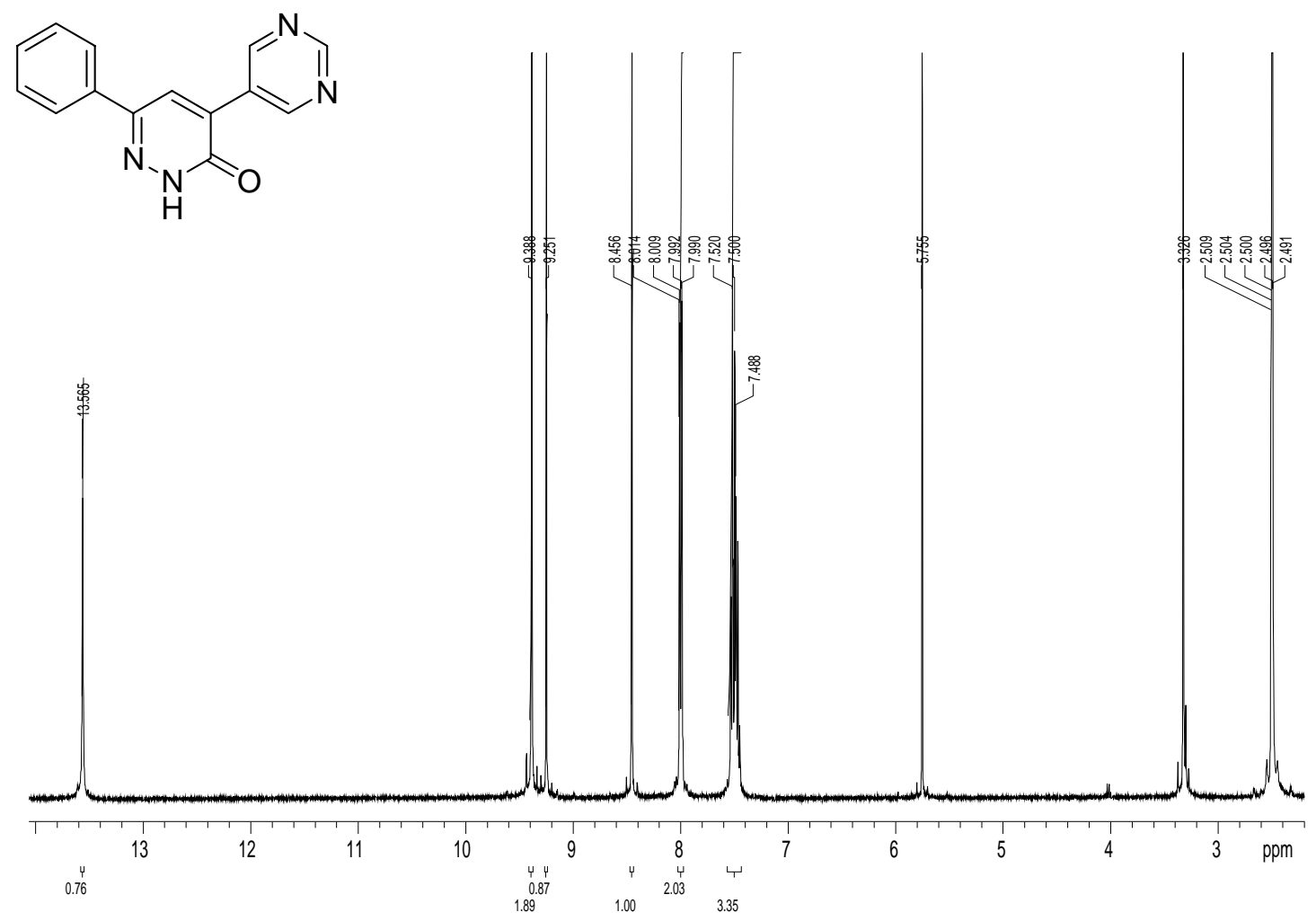

${ }^{13} \mathrm{C}$ NMR (100 MHz, DMSO-d 6 )

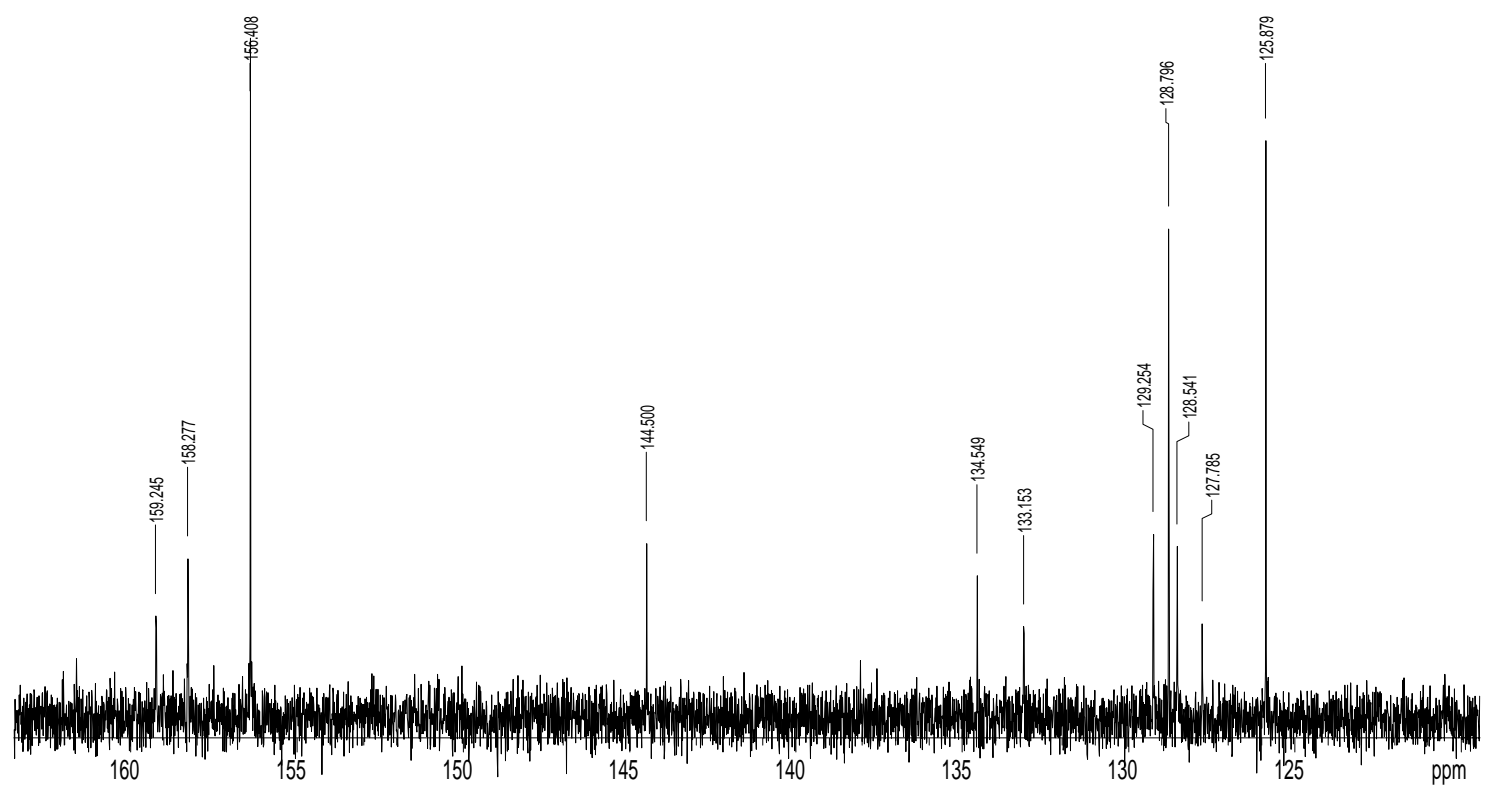

S52 
2-Benzyl-6-phenyl-4-(pyridin-2-yl)pyridazin-3(2H)-one (38)

${ }^{1} \mathrm{H}$ NMR (400 MHz, $\mathrm{CDCl}_{3}$ )

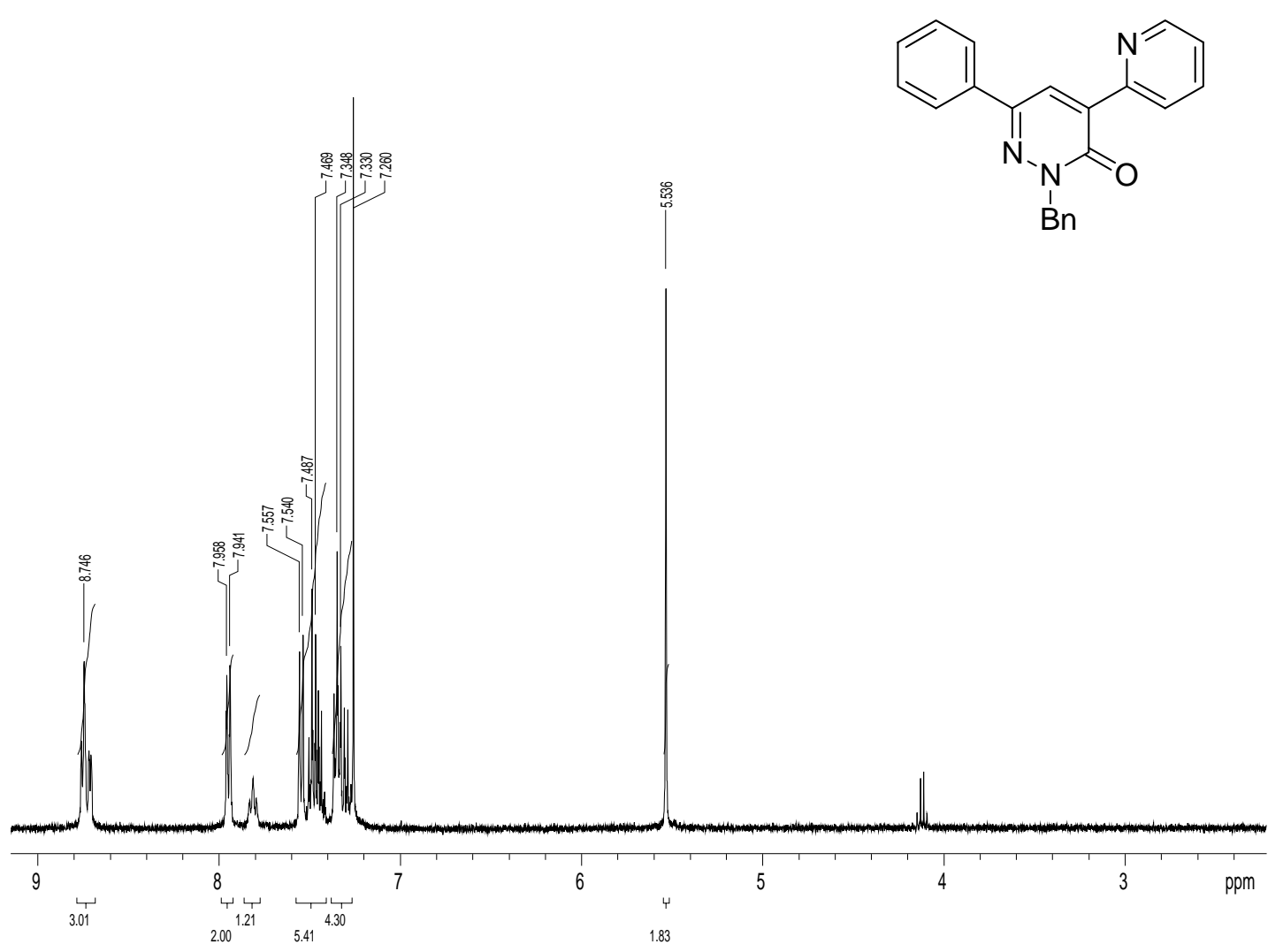

${ }^{13} \mathrm{C}$ NMR (100 MHz, $\mathrm{CDCl}_{3}$ )

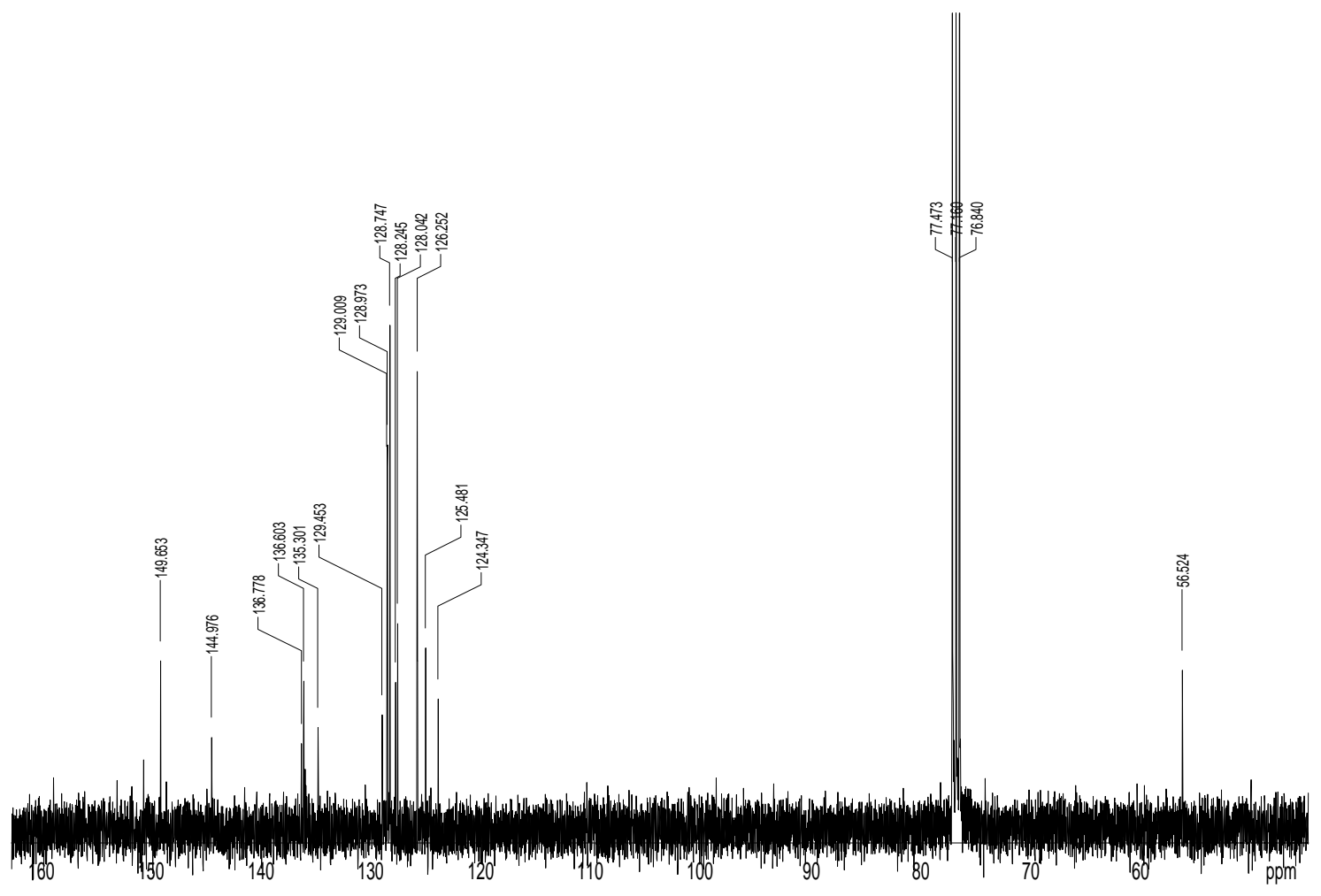




\section{Pyridinium salt 39}

${ }^{1} \mathrm{H}$ NMR (400 MHz, DMSO-d 6 )<smiles>O=c1[nH]nc(-c2ccccc2)cc1-c1cccc[n+]1C(F)(F)F</smiles>

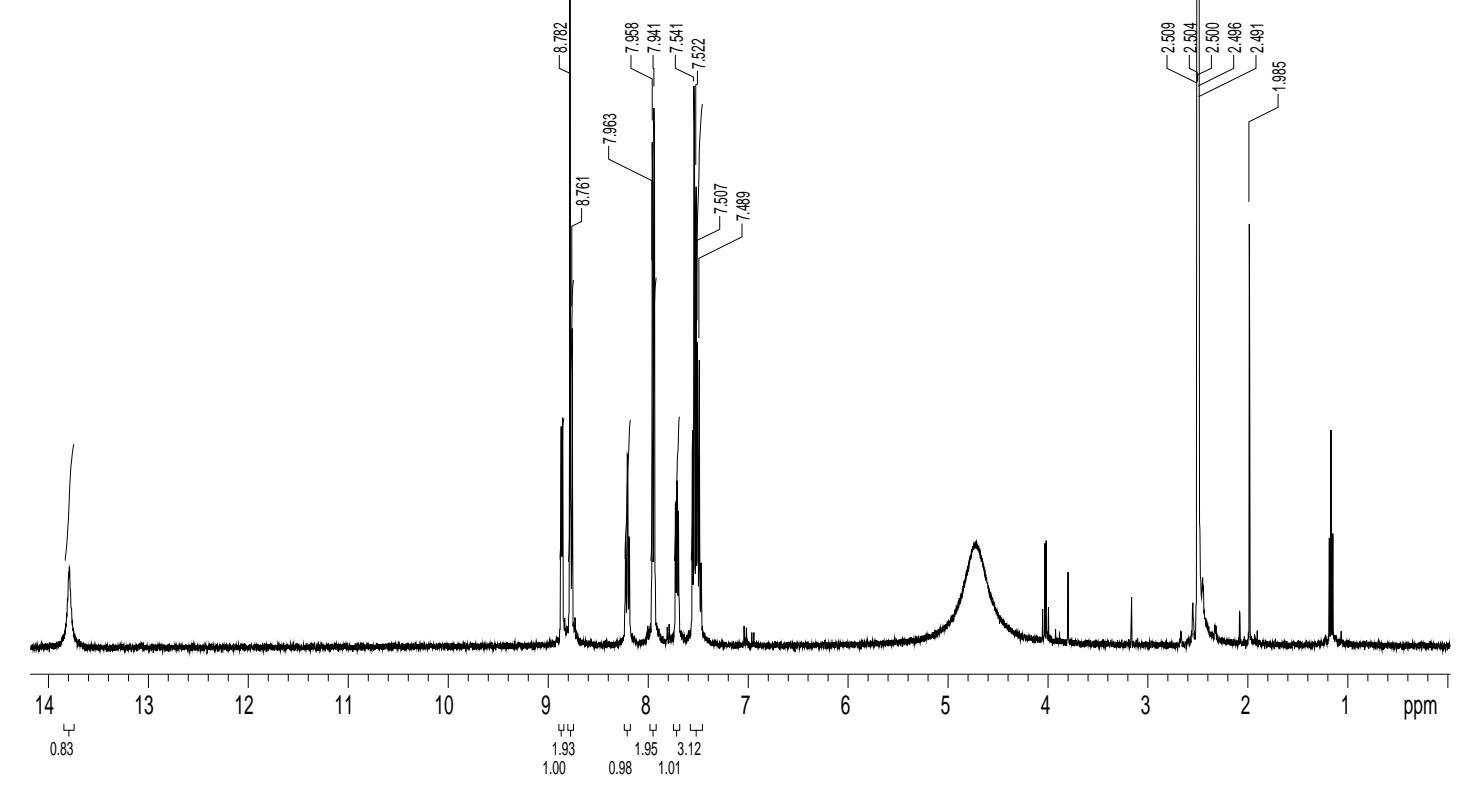

\section{References for the Supporting Information}

(1) Thompson, A. E.; Hughes, G.; Batsanov, A. S.; Bryce, M. R.; Parry, P. R.; Tarbit, B. J. Org. Chem. 2005, 70, 388.

(2) Heck, R. F. in Palladium Reagents in Organic Synthesis, Academic Press, London, 1985, $\mathrm{p} 18$.

(3) Druey, J.; Meier, K.; Eichemberger, K. Helv. Chim. Acta 1954, 37, 121.

(4) Parrot, I; Rival, Y; Wermuth, C.G. Synthesis 1999, 1163.

(5) Draper, T. L.; Bailey, T. R. J. Org. Chem. 1995, 60, 748.

(6) Turck, A.; Plé, N.; Leprêtre-Gaquère, A.; Quéguiner, G. Heterocycles 1998, 49, 205.

(7) Sheldrick, G. M. SHELXTL, version 6.14, Bruker AXS, Madison, WI, USA, 2003.

(8) Gajhede, M.; Larsen, S.; Rettrup, S. Acta Crystallogr. 1986, B42, 545. 